\title{
Fundamental Theorems for the Log Minimal Model Program
}

Dedicated to Professor Shigefumi Mori on the occasion of his sixtieth birthday

\author{
by
}

Osamu FuJino

\begin{abstract}
We prove the cone theorem and the contraction theorem for pairs $(X, B)$, where $X$ is a normal variety and $B$ is an effective $\mathbb{R}$-divisor on $X$ such that $K_{X}+B$ is $\mathbb{R}$-Cartier.

2010 Mathematics Subject Classification: Primary 14E30; Secondary 14C20, 14F17.

Keywords: log minimal model program, log canonical pairs, vanishing theorems, nonvanishing theorem, base point free theorem, rationality theorem, cone theorem, lengths of extremal rays.
\end{abstract}

\section{Contents}

1 Introduction 728

2 Warm-ups 732

3 Kawamata-Viehweg, Nadel, Kollár, ... 734

4 Preliminaries 736

$5 \quad$ Hodge-theoretic injectivity theorem 741

6 Injectivity, torsion-free, and vanishing theorems 744

$7 \quad$ Non-lc ideal sheaves 749

8 Vanishing theorem 750

9 Lc centers 753

10 Dlt blow-ups 754

11 Vanishing theorem for minimal lc centers 758

12 Non-vanishing theorem 759

13 Base point free theorem 763

14 Shokurov's differents 765

15 Rationality theorem 767

16 Cone theorem 771

Communicated by S. Mori. Received November 30, 2009.

O. Fujino: Department of Mathematics, Faculty of Science, Kyoto University, Kyoto 606-8502, Japan;

e-mail: fujino@math.kyoto-u.ac.jp

(C) 2011 Research Institute for Mathematical Sciences, Kyoto University. All rights reserved. 
17 Base point free theorem revisited 777

18 Lengths of extremal rays 778

19 Ambro's theory of quasi-log varieties 785

20 Related topics 786

References 787

\section{$\S 1$. Introduction}

The main purpose of this paper is to prove the following cone and contraction theorem. It is the culmination of the works of several authors: Ambro, Benveniste, Birkar, Kawamata, Kollár, Mori, Reid, Shokurov, and others. It is indispensable for the study of the log minimal model program for varieties with bad singularities (cf. [F17]).

Theorem 1.1 (cf. Theorems 16.4, 16.6, 18.9, and 18.10). Let $X$ be a normal variety defined over $\mathbb{C}$, let $B$ be an effective $\mathbb{R}$-divisor such that $K_{X}+B$ is $\mathbb{R}$-Cartier, and let $\pi: X \rightarrow S$ be a projective morphism onto a variety $S$. Then

$$
\overline{N E}(X / S)=\overline{N E}(X / S)_{K_{X}+B \geq 0}+\overline{N E}(X / S)_{\mathrm{Nlc}(X, B)}+\sum R_{j}
$$

with the following properties:

(1) $\operatorname{Nlc}(X, B)$ is the non-lc locus of $(X, B)$ and

$$
\overline{N E}(X / S)_{\mathrm{Nlc}(X, B)}=\operatorname{Im}(\overline{N E}(\operatorname{Nlc}(X, B) / S) \rightarrow \overline{N E}(X / S)) .
$$

(2) $R_{j}$ is a $\left(K_{X}+B\right)$-negative extremal ray of $\overline{N E}(X / S)$ such that

$$
R_{j} \cap \overline{N E}(X / S)_{\mathrm{Nlc}(X, B)}=\{0\}
$$

for every $j$.

(3) Let $A$ be a $\pi$-ample $\mathbb{R}$-divisor on $X$. Then there are only finitely many $R_{j}$ 's included in $\left(K_{X}+B+A\right)_{<0}$. In particular, the $R_{j}$ 's are discrete in the halfspace $\left(K_{X}+B\right)_{<0}$

(4) Let $F$ be a face of $\overline{N E}(X / S)$ such that

$$
F \cap\left(\overline{N E}(X / S)_{K_{X}+B \geq 0}+\overline{N E}(X / S)_{\mathrm{Nlc}(X, B)}\right)=\{0\} .
$$

Then there exists a contraction morphism $\varphi_{F}: X \rightarrow Y$ over $S$.

(i) Let $C$ be an integral curve on $X$ such that $\pi(C)$ is a point. Then $\varphi_{F}(C)$ is a point if and only if $[C] \in F$.

(ii) $\mathcal{O}_{Y} \simeq\left(\varphi_{F}\right)_{*} \mathcal{O}_{X}$.

(iii) Let $L$ be a line bundle on $X$ such that $L \cdot C=0$ for every curve $C$ with $[C] \in F$. Then there is a line bundle $L_{Y}$ on $Y$ such that $L \simeq \varphi_{F}^{*} L_{Y}$. 
(5) Every $\left(K_{X}+B\right)$-negative extremal ray $R$ with

$$
R \cap \overline{N E}(X / S)_{\mathrm{Nlc}(X, B)}=\{0\}
$$

is spanned by a rational curve $C$ with $0<-\left(K_{X}+B\right) \cdot C \leq 2 \operatorname{dim} X$.

From now on, we further assume that $(X, B)$ is a log canonical pair, that is, $\operatorname{Nlc}(X, B)=\emptyset$. Then we have the following properties:

(6) Let $H$ be an effective $\mathbb{R}$-Cartier $\mathbb{R}$-divisor on $X$ such that $K_{X}+B+H$ is $\pi$-nef and $(X, B+H)$ is log canonical. Then either $K_{X}+B$ is also $\pi$-nef or there is a $\left(K_{X}+B\right)$-negative extremal ray $R$ such that

$$
\left(K_{X}+B+\lambda H\right) \cdot R=0
$$

where

$$
\lambda:=\inf \left\{t \geq 0 \mid K_{X}+B+t H \text { is } \pi-n e f\right\} .
$$

Of course, $K_{X}+B+\lambda H$ is $\pi$-nef.

The first half of Theorem 1.1, that is, (1)-(4), is the main result of [A1]. Ambro's proof depends on the theory of quasi-log varieties. Unfortunately, this theory is inaccessible even to experts because it requires very technical arguments on reducible varieties. In this paper, we give a proof of the above cone and contraction theorem without using the notion of quasi-log varieties. Our approach is much more direct than Ambro's. The reader does not have to refer to [A1] or to the book [F11] in order to read this paper. The latter half of Theorem 1.1, that is, items (5) and (6), is a generalization of the results obtained by Kollár, Kawamata, Shokurov, and Birkar. We note that the formulation of (5) is new. It will play an important role in the log minimal model program with scaling. So, we include it in our cone and contraction theorem.

Here we would like to compare our results with the theory of quasi-log varieties ([A1], [F11]). The first part of Theorem 1.1 was first proved by using the theory of quasi-log varieties; the proof reduced the problem on an irreducible normal variety to one on the union of certain proper closed subvarieties (called non-klt centers) of various dimensions. The notion of quasi-log varieties was a framework to treat such reducible varieties. Thus various strong vanishing theorems on quasilog varieties were needed and a significant part of [F11] was devoted to proving such theorems. Delicate arguments were also needed to overcome several technical difficulties including partial resolutions of reducible closed subvarieties.

The main idea of our paper first appeared in [F16], which treated a special kind of non-klt centers called minimal lc centers and proved their existence and 
normality. The point of our approach was a fully general treatment of minimal lc centers, whose existence and normality were settled earlier under special assumptions.

Vanishing theorems required for these proofs are stronger than the Kawamata -Viehweg-Nadel vanishing theorem but not as difficult as the one on quasi-log varieties. The next step is to reduce the problem on a normal variety to one on its minimal lc centers, where a vanishing theorem plays a central role. Thus it is enough to consider only normal varieties in our treatment. Though we need to prepare vanishing theorems stronger than the Kawamata-Viehweg-Nadel vanishing theorem, they are all proved in our paper (without quoting [A1] or [F11]). In our opinion, the most important contribution of this paper and [F16] is the correct formulation of various vanishing theorems and non-vanishing theorem (Theorem 12.1), from which the cone and contraction theorems can be proved without any difficulties. It is not necessary to treat reducible varieties or precise partial resolutions of singularities of reducible varieties. As already mentioned, the vanishing theorems needed and formulated in our paper are stronger than the Kawamata-Viehweg-Nadel vanishing theorem. It is our belief that this advancement of vanishing theorems distinguishes our treatment from those in [KMM], $[\mathrm{KM}]$ and $[\mathrm{L}]$.

Let us briefly recall the history of the cone and contraction theorem. In the epoch-making paper [Mo], Mori invented the cone theorem for smooth projective varieties and the contraction theorem for smooth projective threefolds. See, for example, [KM, Theorems 1.24 and 1.32]. After Mori's pioneering works, the cone and contraction theorem was proved and generalized for singular varieties by using a completely different method, now called the X-method (cf. [Ka1], [Ko1], [R], and $[\mathrm{S} 1])$. In [A1], Ambro introduced the notion of quasi-log varieties and generalized the cone and contraction theorem. See, for example, [F11, Chapter 3]. For the details of the history of the cone and contraction theorem up to [KMM], we recommend the introductions to Chapters 2, 3, and 4 of [KMM].

We summarize the contents of this paper. Section 2 is a warm-up. Here, we discuss the base point free theorem for projective log canonical surfaces to motivate the reader. This section clarifies the difference between our approach and Ambro's theory of quasi-log varieties. In Section 3, we explain our philosophy on various vanishing theorems. This section helps the reader to understand the subsequent sections. Section 4 collects the preliminary definitions and results. In Section 5, we explain the Hodge-theoretic aspect of the injectivity theorem. It is an easy consequence of the theory of mixed Hodge structures on compact support cohomology groups of smooth quasi-projective varieties. Section 6 treats generalizations of Kollár's injectivity, torsion-free, and vanishing theorems. These 
results play crucial roles in the following sections. They replace the KawamataViehweg vanishing theorem. In Section 7, we introduce the notion of non-lc ideal sheaves. It is an analogue of the well-known multiplier ideal sheaves. Section 8 contains an important vanishing theorem, a generalization of the Nadel vanishing theorem. It is very useful for the study of log canonical pairs. In Section 9 , we recall the basic properties of lc centers. Section 10 treats the dlt blow-up following Hacon and its slight refinement, which will be useful for future studies (cf. [G]). Here, we need [BCHM]. In Section 11, we give a vanishing theorem for minimal lc centers. By the dlt blow-up obtained in Section 10, we can easily prove our important vanishing theorem. Section 12 is devoted to the proof of the non-vanishing theorem. In Section 13, we prove the base point free theorem. It is a direct consequence of the non-vanishing theorem. In Section 14, we quickly recall Shokurov's differents. Section 15 is devoted to the rationality theorem. In Section 16, we obtain the cone theorem and contraction theorem by using the rationality theorem and the base point free theorem. Section 17 is a supplement to the base point free theorem. In Section 18, we discuss estimates of lengths of extremal rays. It is important for the study of the log minimal model program with scaling. Our formulation for non-lc pairs is new. In Section 19, we quickly explain some results which were obtained by the theory of quasi-log varieties but cannot be covered by our new approach. In the final Section 20, we briefly discuss some related topics considered by the author in other publications.

This paper grew out of the ideas in [F16]. The result in Section 10 heavily depends on $[\mathrm{BCHM}]$. We use it to prove the vanishing theorem for minimal lc centers in Section 11. We note that the result in Section 11 can be proved without applying [BCHM] if we discuss the theory of mixed Hodge structures on compact support cohomology groups of reducible varieties. This was carried out in [F11, Chapter 2]. We note that [F11, Chapter 2] is independent of the log minimal model program for klt pairs. So, the non-vanishing theorem (Theorem 12.1), the base point free theorem (Theorem 13.1), the rationality theorem (Theorem 15.1), and the cone theorem (Theorem 16.6) do not depend on the corresponding results for klt pairs. Therefore, our proofs are new even for klt pairs. In Section 18, we need Theorem 10.4, which is a consequence of [BCHM], to prove Theorems 18.2 and 18.10. At present there are no proofs of Theorems 18.2 and 18.10 without using [BCHM]. However, Theorem 18.2 can be directly proved if we have an appropriate vanishing theorem for projective morphisms between analytic spaces. For the details, see [F11, Remark 3.22].

We will work over $\mathbb{C}$, the complex number field, throughout this paper. 


\section{§2. Warm-ups}

In this section, we explain the base point free theorem for projective log canonical surfaces to motivate the reader. This section clarifies the difference between our new approach and Ambro's theory of quasi-log varieties. We refer the reader to [F15, Section 4] for Ambro's approach. The following theorem is a very special case of Theorem 13.1.

Theorem 2.1 (Base point free theorem for lc surfaces). Let $(X, B)$ be a projective log canonical surface. Let $L$ be a nef Cartier divisor on $X$ such that aL$\left(K_{X}+B\right)$ is ample for some $a>0$. Then $|m L|$ is base point free for $m \gg 0$.

This cannot be proved by the traditional X-method. A key ingredient of this paper is the following generalization of Kollár's vanishing theorem. We will describe it in Section 8.

Theorem 2.2 (cf. Theorem 8.1). Let $(X, B)$ be a projective log canonical pair. Let $D$ be a Cartier divisor on $X$ such that $D-\left(K_{X}+B\right)$ is ample. Let $C$ be an lc center of $(X, B)$ with the reduced scheme structure. Then

$$
H^{i}\left(X, \mathcal{I}_{C} \otimes \mathcal{O}_{X}(D)\right)=0 \quad \text { for every } i>0,
$$

where $\mathcal{I}_{C}$ is the defining ideal sheaf of $C$. In particular, the restriction map $H^{0}\left(X, \mathcal{O}_{X}(D)\right) \rightarrow H^{0}\left(C, \mathcal{O}_{C}(D)\right)$ is surjective.

In Theorem 2.2, we do not assume that $C$ is isolated in the non-klt locus of the pair $(X, B)$; nor do we assume that there exists another boundary $\mathbb{R}$-divisor $B^{\prime}$ on $X$ such that $\left(X, B^{\prime}\right)$ is klt. Therefore, the theorem cannot be proved by the traditional arguments depending on the Kawamata-Viehweg-Nadel vanishing theorem.

The next theorem is a special case of Theorem 12.1. This formulation was first introduced in [F16]. We will see that it is equivalent to Theorem 2.1.

Theorem 2.3 (Non-vanishing theorem for lc surfaces). Let $X$ be a projective log canonical surface. Let $L$ be a nef Cartier divisor on $X$ such that $a L-\left(K_{X}+B\right)$ is ample for some $a>0$. Then the base locus $\mathrm{Bs}|m L|$ of $|m L|$ contains no lc centers of $(X, B)$ for $m \gg 0$.

Proof. It is sufficient to check that $\mathrm{Bs}|m L|$ contains no minimal lc centers of $(X, B)$ for $m \gg 0$. Let $C$ be a minimal lc center of $(X, B)$. If $C$ is a point $P$, then Bs $|m L|$ does not contain $C$ for every $m \geq a$, because the evaluation map

$$
H^{0}\left(X, \mathcal{O}_{X}(m L)\right) \rightarrow \mathbb{C}(P) \simeq H^{0}\left(P, \mathcal{O}_{P}(m L)\right)
$$


is surjective for every $m \geq a$ by Theorem 2.2. If $C$ is a curve, then $C \subset\llcorner B\lrcorner$ and $(X, B)$ is plt around $C$. Therefore,

$$
K_{C}+B_{C}=\left.\left(K_{X}+B\right)\right|_{C}
$$

is klt by adjunction. Since $\left.a L\right|_{C}-\left(K_{C}+B_{C}\right)$ is ample, there exists $m_{1}$ such that $|m L|_{C} \mid$ is base point free for every $m \geq m_{1}$. By Theorem 2.2, the restriction map

$$
H^{0}\left(X, \mathcal{O}_{X}(m L)\right) \rightarrow H^{0}\left(C, \mathcal{O}_{C}(m L)\right)
$$

is surjective for every $m \geq a$. Thus, Bs $|m L|$ does not contain $C$ for $m \gg 0$. So, the proof is finished since there are only finitely many minimal lc centers.

In the above proof, $C$ is a point or a divisor on $X$. So, there are no difficulties in investigating minimal lc centers. When $\operatorname{dim} X \geq 3$, we need a more powerful vanishing theorem (cf. Theorem 11.1) to study linear systems on minimal lc centers.

Let us explain the proof of Theorem 2.1.

Proof of Theorem 2.1. If $(X, B)$ is klt, then the statement is well-known as the Kawamata-Shokurov base point free theorem (cf. [KM, Theorem 3.3]). So, we assume that $(X, B)$ is lc but not klt for simplicity. By Theorem 2.3, we can take general members $D_{1}, D_{2}, D_{3} \in\left|m_{1} L\right|$ for some $m_{1}>0$. If $\operatorname{Bs}\left|m_{1} L\right|=\emptyset$, then $L$ is semi-ample. Therefore, we assume that $\operatorname{Bs}\left|m_{1} L\right| \neq \emptyset$. We note that $(X, B+D)$, where $D=D_{1}+D_{2}+D_{3}$, is $\log$ canonical outside Bs $\left|m_{1} L\right|$, and $(X, B+D)$ is not $\log$ canonical at the generic point of every irreducible component of $\operatorname{Bs}\left|m_{1} L\right|$. Let $c$ be the $\log$ canonical threshold of $(X, B)$ with respect to $D$. Then $c>0$ by Theorem 2.3, and $c<1$ because $(X, B+D)$ is not $\log$ canonical. By the above construction, $(X, B+c D)$ is $\log$ canonical and there is an lc center $C$ of $(X, B+c D)$ such that $C$ is contained in $\operatorname{Bs}\left|m_{1} L\right|$. By applying Theorem 2.3 to

$$
\left(3 c m_{1}+a\right) L-\left(K_{X}+B+c D\right) \sim_{\mathbb{Q}} a L-\left(K_{X}+B\right)
$$

on $(X, B+c D)$, we see that $\mathrm{Bs}\left|m_{2} m_{1} L\right|$ does not contain $C$ for $m_{2} \gg 0$. Therefore, $\mathrm{Bs}\left|m_{2} m_{1} L\right| \subsetneq \mathrm{Bs}\left|m_{1} L\right|$. By noetherian induction, we find that $L$ is semi-ample. With a little care, we can check that $|m L|$ is base point free for $m \gg 0$. We omit some details here. For the details, see the proof of Theorem 13.1.

In Ambro's framework of quasi-log varieties (cf. [A1], [F11], and [F15]), we have to discuss the base point free theorem for certain reducible curves (cf. [F1]) to prove Theorem 2.1. We note that the ultimate generalization of Theorem 2.1 for surfaces is proved in [F17]. 
One of the main purposes of this paper is to generalize Theorem 2.3 to pairs $(X, B)$, where $X$ is an $n$-dimensional normal variety and $B$ is an effective $\mathbb{R}$-divisor on $X$ such that $K_{X}+B$ is $\mathbb{R}$-Cartier (see Theorems 12.1 and 13.1).

\section{§3. Kawamata-Viehweg, Nadel, Kollár, ...}

In this section, we explain our philosophy on vanishing theorems. There is a big conceptual difference between our approach in this paper and the traditional arguments based on the Kawamata-Viehweg-Nadel vanishing theorem (cf. [KMM], $[\mathrm{KM}]$, and $[\mathrm{L}])$.

In the traditional X-method, the following type of the Kawamata-Viehweg vanishing theorem plays a crucial role (cf. [KM, Theorem 3.1], [L, Theorem 9.1.18]).

Theorem 3.1 (The Kawamata-Viehweg vanishing theorem). Let $X$ be a smooth projective variety and let $B$ be an effective $\mathbb{Q}$-divisor such that $\operatorname{Supp} B$ is simple normal crossing and $\llcorner B\lrcorner=0$. Let $L$ be a Cartier divisor on $X$ such that $L-$ $\left(K_{X}+B\right)$ is nef and big. Then

$$
H^{i}\left(X, \mathcal{O}_{X}(L)\right)=0 \quad \text { for every } i>0 .
$$

Recently, the (algebraic version of) Nadel vanishing theorem, which is a generalization of the above Kawamata-Viehweg vanishing theorem, is very often used for the study of linear systems (cf. [L, Theorem 9.4.17]).

Theorem 3.2 (The Nadel vanishing theorem). Let $X$ be a normal projective variety and let $B$ be an effective $\mathbb{Q}$-divisor on $X$ such that $K_{X}+B$ is $\mathbb{Q}$-Cartier. Let $L$ be a Cartier divisor on $X$ such that $L-\left(K_{X}+B\right)$ is nef and big. Then

$$
H^{i}\left(X, \mathcal{O}_{X}(L) \otimes \mathcal{J}(X, B)\right)=0 \quad \text { for every } i>0,
$$

where $\mathcal{J}(X, B)$ is the multiplier ideal sheaf of $(X, B)$ (see Remark 7.3 below).

The following relative version of the Kawamata-Viehweg vanishing theorem sometimes plays an important role implicitly (cf. [L, Theorem 9.4.17], [KM, Corollary 2.68]).

Theorem 3.3 (The relative Kawamata-Viehweg vanishing theorem). Let $X$ be a normal projective variety and let $B$ be an effective $\mathbb{Q}$-divisor on $X$ such that $K_{X}+B$ is $\mathbb{Q}$-Cartier. Let $f: Y \rightarrow X$ be a projective resolution such that $K_{Y}+B_{Y}=$ $f^{*}\left(K_{X}+B\right)$ and $\operatorname{Supp} B_{Y}$ is simple normal crossing. Then

$$
R^{i} f_{*} \mathcal{O}_{Y}\left(-\left\llcorner B_{Y}\right\lrcorner\right)=0 \quad \text { for every } i>0 .
$$


It is obvious that 3.1 is a special case of 3.2 . It is a routine exercise to deduce 3.3 from 3.1. We note that 3.2 can be obtained as a consequence of 3.1 and 3.3 by Hironaka's resolution theorem and Leray's spectral sequence. In this paper, we see the Nadel vanishing theorem 3.2 (resp. the relative Kawamata-Viehweg vanishing theorem 3.3) as a special case of Kollár's vanishing theorem 3.4(ii) (resp. Kollár's torsion-free theorem 3.4(i)).

Let us recall Kollár's theorems (cf. [Ko4, 10.15 Corollary]).

Theorem 3.4 (Kollár's torsion-free and vanishing theorems). Let $Y$ be a smooth projective variety and let $\Delta$ be an effective $\mathbb{Q}$-divisor on $Y$ such that $\operatorname{Supp} \Delta$ is simple normal crossing and $\llcorner\Delta\lrcorner=0$. Let $f: Y \rightarrow X$ be a surjective morphism onto a projective variety $X$ and let $D$ be a Cartier divisor on $Y$.

(i) If $D-\left(K_{Y}+\Delta\right) \sim_{\mathbb{Q}} f^{*} M$ for some $\mathbb{Q}$-Cartier $\mathbb{Q}$-divisor $M$ on $X$, then $R^{i} f_{*} \mathcal{O}_{Y}(D)$ is torsion-free for every $i \geq 0$. In particular, $R^{i} f_{*} \mathcal{O}_{Y}(D)=0$ for every $i>0$ if $f$ is birational.

(ii) If $D-\left(K_{Y}+\Delta\right) \sim_{\mathbb{Q}} f^{*} M$, where $M$ is an ample $\mathbb{Q}$-divisor on $X$, then

$$
H^{i}\left(X, R^{j} f_{*} \mathcal{O}_{Y}(D)\right)=0 \quad \text { for every } i>0 \text { and } j \geq 0 \text {. }
$$

We will completely generalize it in Theorem 6.3. As we stated above, in this paper, 3.2 is not seen as a combination of 3.1 and 3.3. It should be recognized as a special case of Kollár's vanishing theorem 3.4(ii). We do not see the vanishing theorem 3.3 as a relative vanishing theorem but as a special case of Kollár's torsionfree theorem 3.4(i). This change of viewpoint opens the door to the study of log canonical pairs.

3.5 (Philosophy). We note that 3.4 follows from the theory of pure Hodge structures. In our philosophy, we have the following correspondences.

$$
\text { Kawamata log terminal pairs } \Longleftrightarrow \text { Pure Hodge structures }
$$

and

$$
\text { Log canonical pairs } \Longleftrightarrow \quad \text { Mixed Hodge structures }
$$

Therefore, it is very natural to prove a "mixed" version of 3.4 for the study of $\log$ canonical pairs. We will carry it out in Sections 5 and 6 . There is a big difference between our framework discussed in this paper (cf. Sections 12, 13, and 15) and the traditional X-method from the Hodge-theoretic viewpoint. We believe 
that all the results for klt pairs can be proved without using the theory of mixed Hodge structures (cf. [F14]).

3.6 (Further discussions). When we consider various extension theorems, which play a crucial role in the proof of the existence of pl flips (cf. [HM]), we think that the following correspondence is natural.

$$
\text { Kawamata log terminal pairs } \Longleftrightarrow L^{2} \text {-method }
$$

The extension theorem in $[\mathrm{HM}]$ can be proved as a consequence of the usual vanishing theorems. However, we note that the origin of the extension theorem is the Ohsawa-Takegoshi $L^{2}$ extension theorem. The Nadel vanishing theorem also has its origin in the $L^{2}$-method. It is very natural to try to generalize the above correspondence for log canonical pairs. However, we do not know what should be in the right box in the correspondence below.

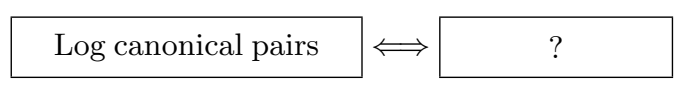

Here, we do not discuss this topic any more.

\section{$\S 4$. Preliminaries}

We work over the complex number field $\mathbb{C}$ throughout this paper. But we note that by using the Lefschetz principle, we can extend almost everything to the case where the base field is an algebraically closed field of characteristic zero. In this paper, an algebraic scheme denotes a scheme which is separated and of finite type over $\mathbb{C}$. We collect the basic notation and definitions.

$4.1(m \gg 0)$. The expression '.. for $m \gg 0$ ' means that 'there exists a positive number $m_{0}$ such that ... for every $m \geq m_{0}$.'

4.2 (Operations on $\mathbb{R}$-divisors). For an $\mathbb{R}$-Weil divisor $D=\sum_{j=1}^{r} d_{j} D_{j}$ such that $D_{j}$ is a prime divisor for every $j$ and $D_{i} \neq D_{j}$ for $i \neq j$, we define the roundup $\ulcorner D\urcorner=\sum_{j=1}^{r}\left\ulcorner d_{j}\right\urcorner D_{j}$ (resp. the round-down $\llcorner D\lrcorner=\sum_{j=1}^{r}\left\llcorner d_{j}\right\lrcorner D_{j}$ ), where for every real number $x,\ulcorner x\urcorner$ (resp. $\llcorner x\lrcorner$ ) is the integer defined by $x \leq\ulcorner x\urcorner<x+1$ (resp. $x-1<\llcorner x\lrcorner \leq x)$. The fractional part $\{D\}$ of $D$ denotes $D-\llcorner D\lrcorner$. We define

$$
D^{=1}=\sum_{d_{j}=1} D_{j}, \quad D^{\leq 1}=\sum_{d_{j} \leq 1} d_{j} D_{j}, \quad D^{<1}=\sum_{d_{j}<1} d_{j} D_{j}, \quad D^{>1}=\sum_{d_{j}>1} d_{j} D_{j} .
$$

We call $D$ a boundary $\mathbb{R}$-divisor if $0 \leq d_{j} \leq 1$ for every $j$. We note that $\sim_{\mathbb{Q}}$ (resp. $\sim_{\mathbb{R}}$ ) denotes the $\mathbb{Q}$-linear (resp. $\mathbb{R}$-linear) equivalence of $\mathbb{Q}$-divisors (resp. 
$\mathbb{R}$-divisors). Let $D_{1}$ and $D_{2}$ be $\mathbb{R}$-Cartier $\mathbb{R}$-divisors on $X$ and let $f: X \rightarrow Y$ be a morphism. We say that $D_{1}$ and $D_{2}$ are $\mathbb{R}$-linearly $f$-equivalent, denoted by $D_{1} \sim_{\mathbb{R}, f} D_{2}$, if there is an $\mathbb{R}$-Cartier $\mathbb{R}$-divisor $B$ on $Y$ such that $D_{1} \sim_{\mathbb{R}} D_{2}+f^{*} B$. We can define $D_{1} \sim_{\mathbb{Q}, f} D_{2}$ for $\mathbb{Q}$-Cartier $\mathbb{Q}$-divisors $D_{1}$ and $D_{2}$ similarly.

Definition 4.3 (Exceptional locus). For a proper birational morphism $f$ : $X \rightarrow Y$, the exceptional locus $\operatorname{Exc}(f) \subset X$ is the locus where $f$ is not an isomorphism.

4.4 (Discrepancy, singularities of pairs, etc.). Let $X$ be a normal variety and let $B$ be an effective $\mathbb{R}$-divisor on $X$ such that $K_{X}+B$ is $\mathbb{R}$-Cartier. Let $f: Y \rightarrow X$ be a resolution such that $\operatorname{Exc}(f) \cup f_{*}^{-1} B$ has a simple normal crossing support, where $f_{*}^{-1} B$ is the strict transform of $B$ on $Y$. We write

$$
K_{Y}=f^{*}\left(K_{X}+B\right)+\sum_{i} a_{i} E_{i}
$$

and $a\left(E_{i}, X, B\right)=a_{i}$. We say that $(X, B)$ is $l c$ (resp. $\left.k l t\right)$ if $a_{i} \geq-1$ (resp. $\left.a_{i}>-1\right)$ for every $i$. Note that the discrepancy $a(E, X, B) \in \mathbb{R}$ can be defined for every prime divisor $E$ over $X$. If $a(E, X, B)>-1$ for every exceptional divisor $E$ over $X$, then the pair $(X, B)$ is called plt. Here, lc (resp. klt, plt) is an abbreviation of $\log$ canonical (resp. Kawamata log terminal, purely log terminal). By the definition, there exists the largest Zariski open set $U$ (resp. $U^{\prime}$ ) of $X$ such that $(X, B)$ is lc (resp. klt) on $U$ (resp. $\left.U^{\prime}\right)$. We put $\operatorname{Nlc}(X, B)=X \backslash U\left(\operatorname{resp} . \operatorname{Nklt}(X, B)=X \backslash U^{\prime}\right)$ and call it the non-lc locus (resp. non-klt locus) of the pair $(X, B)$. We sometimes simply denote $\operatorname{Nlc}(X, B)$ by $X_{\mathrm{NLC}}$.

Let $(X, B)$ be a log canonical pair and let $M$ be an effective $\mathbb{R}$-Cartier $\mathbb{R}$ divisor on $X$. The $\log$ canonical threshold of $(X, B)$ with respect to $M$ is defined by

$$
c=\sup \{t \in \mathbb{R} \mid(X, B+t M) \text { is } \log \text { canonical }\} .
$$

Definition 4.5 (Center). Let $E$ be a prime divisor over $X$. The closure of the image of $E$ on $X$ is denoted by $c_{X}(E)$ and called the center of $E$ on $X$.

Definition 4.6 (Lc center). Let $X$ be a normal variety and let $B$ be an effective $\mathbb{R}$-divisor on $X$ such that $K_{X}+B$ is $\mathbb{R}$-Cartier. If $a(E, X, B)=-1$ and $c_{X}(E)$ is not contained in $\operatorname{Nlc}(X, B)$, then $c_{X}(E)$ is called an lc center of $(X, B)$. It is obvious that there are at most finitely many lc centers.

We note that our definition of lc centers is slightly different from the usual one. For details, see [FST, Section 3]. 
Definition 4.7 (Stratum). Let $(X, B)$ be a log canonical pair. A stratum of $(X, B)$ is $X$ itself or an lc center of $(X, B)$.

Let $T$ be a simple normal crossing divisor on a smooth variety $Y$. A stratum of $T$ is a stratum of the pair $(Y, T)$ contained in $T$.

4.8 (Kleiman-Mori cone). Let $X$ be an algebraic scheme over $\mathbb{C}$ and let $\pi: X \rightarrow S$ be a proper morphism to an algebraic scheme $S$. Let $\operatorname{Pic}(X)$ be the group of line bundles on $X$. Take a complete curve on $X$ which is mapped to a point by $\pi$. For $\mathcal{L} \in \operatorname{Pic}(X)$, we define the intersection number $\mathcal{L} \cdot C=\operatorname{deg}_{\bar{C}} f^{*} \mathcal{L}$, where $f: \bar{C} \rightarrow C$ is the normalization of $C$. Via this intersection pairing, we introduce a bilinear form

$$
\cdot: \operatorname{Pic}(X) \times Z_{1}(X / S) \rightarrow \mathbb{Z}
$$

where $Z_{1}(X / S)$ is the free abelian group generated by integral curves which are mapped to points on $S$ by $\pi$.

Now we have the notion of numerical equivalence both in $Z_{1}(X / S)$ and in $\operatorname{Pic}(X)$, which is denoted by $\equiv$, and we obtain a perfect pairing

$$
N^{1}(X / S) \times N_{1}(X / S) \rightarrow \mathbb{R}
$$

where

$$
N^{1}(X / S)=\{\operatorname{Pic}(X) / \equiv\} \otimes \mathbb{R} \quad \text { and } \quad N_{1}(X / S)=\left\{Z_{1}(X / S) / \equiv\right\} \otimes \mathbb{R}
$$

that is, $N^{1}(X / S)$ and $N_{1}(X / S)$ are dual to each other through this intersection pairing. It is well known that

$$
\operatorname{dim}_{\mathbb{R}} N^{1}(X / S)=\operatorname{dim}_{\mathbb{R}} N_{1}(X / S)<\infty .
$$

We write $\rho(X / S)=\operatorname{dim}_{\mathbb{R}} N^{1}(X / S)=\operatorname{dim}_{\mathbb{R}} N_{1}(X / S)$. We define the KleimanMori cone $\overline{N E}(X / S)$ as the closed convex cone in $N_{1}(X / S)$ generated by integral curves on $X$ which are mapped to points on $S$ by $\pi$. When $S=\operatorname{Spec} \mathbb{C}$, we drop /Spec $\mathbb{C}$ from the notation, e.g., we simply write $N_{1}(X)$ instead of $N_{1}(X / \operatorname{Spec} \mathbb{C})$.

Definition 4.9. An element $D \in N^{1}(X / S)$ is called $\pi$-nef (or relatively nef for $\pi$ ) if $D \geq 0$ on $\overline{N E}(X / S)$. When $S=$ Spec $\mathbb{C}$, we simply say that $D$ is nef.

Theorem 4.10 (Kleiman's criterion for ampleness). Let $\pi: X \rightarrow S$ be a projective morphism between algebraic schemes. Then $\mathcal{L} \in \operatorname{Pic}(X)$ is $\pi$-ample if and only if the numerical class of $\mathcal{L}$ in $N^{1}(X / S)$ gives a positive function on $\overline{N E}(X / S) \backslash\{0\}$.

In Theorem 4.10, we note that the projectivity of $\pi$ is indispensable (cf. [F4]). 
Definition 4.11 (Semi-ample $\mathbb{R}$-divisors). An $\mathbb{R}$-Cartier $\mathbb{R}$-divisor $D$ on $X$ is $\pi$ semi-ample if $D \sim_{\mathbb{R}} \sum_{i} a_{i} D_{i}$, where $D_{i}$ is a $\pi$-semi-ample Cartier divisor on $X$ and $a_{i}$ is a positive real number for every $i$.

Remark 4.12. In Definition 4.11, we can replace $D \sim_{\mathbb{R}} \sum_{i} a_{i} D_{i}$ with $D=$ $\sum_{i} a_{i} D_{i}$ since every principal Cartier divisor on $X$ is $\pi$-semi-ample.

The following two lemmas seem to be missing in the literature.

Lemma 4.13. Let $D$ be an $\mathbb{R}$-Cartier $\mathbb{R}$-divisor on $X$. Then the following conditions are equivalent:

(1) $D$ is $\pi$-semi-ample.

(2) There exists a morphism $f: X \rightarrow Y$ over $S$ such that $D \sim_{\mathbb{R}} f^{*} A$, where $A$ is an $\mathbb{R}$-Cartier $\mathbb{R}$-divisor on $Y$ which is ample over $S$.

Proof. It is obvious that (1) follows from (2). If $D$ is $\pi$-semi-ample, then we can write $D \sim_{\mathbb{R}} \sum_{i} a_{i} D_{i}$ as in Definition 4.11. By replacing $D_{i}$ with its multiple, we can assume that $\pi^{*} \pi_{*} \mathcal{O}_{X}\left(D_{i}\right) \rightarrow \mathcal{O}_{X}\left(D_{i}\right)$ is surjective for every $i$. Let $f: X \rightarrow Y$ be a morphism over $S$ obtained from the surjection $\pi^{*} \pi_{*} \mathcal{O}_{X}\left(\sum_{i} D_{i}\right) \rightarrow \mathcal{O}_{X}\left(\sum_{i} D_{i}\right)$. Then it is easy to see that $f: Y \rightarrow X$ has the desired property.

Lemma 4.14. Let $D$ be a Cartier divisor on $X$. If $D$ is $\pi$-semi-ample in the sense of Definition 4.11, then $D$ is $\pi$-semi-ample in the usual sense, that is, $\pi^{*} \pi_{*} \mathcal{O}_{X}(m D) \rightarrow \mathcal{O}_{X}(m D)$ is surjective for some positive integer $m$. In particular, Definition 4.11 is reasonable.

Proof. We write $D \sim_{\mathbb{R}} \sum_{i} a_{i} D_{i}$ as in Definition 4.11. Let $f: X \rightarrow Y$ be a morphism in Lemma 4.13(2). By taking the Stein factorization, we can assume that $f$ has connected fibers. By the construction, $D_{i} \sim_{\mathbb{Q}, f} 0$ for every $i$. By replacing $D_{i}$ with its multiple, we can assume that $D_{i} \sim f^{*} D_{i}^{\prime}$ for some Cartier divisor $D_{i}^{\prime}$ on $Y$ for every $i$. Let $U$ be any Zariski open set of $Y$ on which $D_{i}^{\prime} \sim 0$ for every $i$. On $f^{-1}(U)$, we have $D \sim_{\mathbb{R}} 0$. This implies $D \sim_{\mathbb{Q}} 0$ on $f^{-1}(U)$ since $D$ is Cartier. Therefore, there exists a positive integer $m$ such that $f^{*} f_{*} \mathcal{O}_{X}(m D) \rightarrow \mathcal{O}_{X}(m D)$ is surjective. By this surjection, we have $m D \sim f^{*} A$ for a Cartier divisor $A$ on $Y$ which is ample over $S$. This means that $D$ is $\pi$-semi-ample in the usual sense.

We will repeatedly use the following easy lemma. We give a detailed proof for the reader's convenience.

Lemma 4.15. Let $X$ be a normal variety and let $B$ be an effective $\mathbb{R}$-Cartier $\mathbb{R}$ divisor on $X$ such that $\llcorner B\lrcorner=0$. Let $A$ be a Cartier divisor on $X$. Assume that 
$A \sim_{\mathbb{R}} B$. Then there exists a $\mathbb{Q}$-Cartier $\mathbb{Q}$-divisor $C$ on $X$ such that $A \sim_{\mathbb{Q}} C$, $\llcorner C\lrcorner=0$, and $\operatorname{Supp} C=\operatorname{Supp} B$.

Proof. We can write $B=A+\sum_{i=1}^{k} r_{i}\left(f_{i}\right)$, where $r_{i} \in \mathbb{R}$ and $f_{i}$ is a rational function on $X$ for every $i$. We put

$$
E=\operatorname{Supp} A \cup \operatorname{Supp} B \cup \bigcup_{i=1}^{k} \operatorname{Supp}\left(f_{i}\right) \text {. }
$$

Let $E=\sum_{j=1}^{n} E_{j}$ be the irreducible decomposition of $E$. We can write

$$
A=\sum_{j} a_{j} E_{j}, \quad B=\sum_{j} b_{j} E_{j},
$$

and

$$
\left(f_{i}\right)=\sum_{j} m_{i j} E_{j} \quad \text { for every } i .
$$

We can assume that $b_{j} \in \mathbb{Q}$ for $1 \leq j \leq l$ and $b_{j} \notin \mathbb{Q}$ for $l+1 \leq j \leq n$. We note that $a_{j} \in \mathbb{Z}$ for every $j$ and that $m_{i j} \in \mathbb{Z}$ for every $i, j$. We define

$$
\mathcal{S}=\left\{\left(v_{1}, \ldots, v_{k}\right) \in \mathbb{R}^{k} \mid b_{j}=a_{j}+\sum_{i=1}^{k} v_{i} m_{i j} \text { for } 1 \leq j \leq l\right\} .
$$

Then $\mathcal{S}$ is an affine subspace of $\mathbb{R}^{k}$ defined over $\mathbb{Q}$. We note that $\mathcal{S}$ is not empty since $\left(r_{1}, \ldots, r_{k}\right) \in \mathcal{S}$. If we take $\left(r_{1}^{\prime}, \ldots, r_{k}^{\prime}\right) \in \mathcal{S} \cap \mathbb{Q}^{k}$ which is very close to $\left(r_{1}, \ldots, r_{k}\right)$ and put $C=A+\sum_{i} r_{i}^{\prime}\left(f_{i}\right)$, then it is obvious that $C$ has the desired properties.

The next lemma is well known as the negativity lemma.

Lemma 4.16 (Negativity lemma). Let $h: Z \rightarrow Y$ be a proper birational morphism between normal varieties. Let $-B$ be an h-nef $\mathbb{R}$-Cartier $\mathbb{R}$-divisor on $Z$. Then we have the following statements:

(1) $B$ is effective if and only if $h_{*} B$ is.

(2) Assume that $B$ is effective. Then for every $y \in Y$, either $h^{-1}(y) \subset \operatorname{Supp} B$ or $h^{-1}(y) \cap \operatorname{Supp} B=\emptyset$.

Lemma 4.16 is essentially an application of the Hodge index theorem for smooth projective surfaces. For the proof, see, for example, [KM, Lemma 3.39].

We close this section with the following useful lemma. It is a consequence of Szabó's resolution lemma. 
Lemma 4.17. Let $Z$ be a smooth variety and let $B$ be an $\mathbb{R}$-divisor on $Z$ such that $\operatorname{Supp} B$ is simple normal crossing. Let $f: Z \rightarrow X$ be a projective morphism and let $\bar{X}$ be a projective variety such that $\bar{X}$ contains $X$ as a Zariski open set. Then there exist a smooth projective variety $\bar{Z}$ and an $\mathbb{R}$-divisor $\bar{B}$ on $\bar{Z}$ such that

(i) $f: Z \rightarrow X$ can be extended to $\bar{f}: \bar{Z} \rightarrow \bar{X}$.

(ii) Supp $\bar{B}$ is simple normal crossing.

(iii) Supp $\bar{B} \cup \operatorname{Supp}(\bar{Z} \backslash Z)$ is simple normal crossing.

(iv) $\left.\bar{B}\right|_{Z}=B$.

Proof. Let $Z^{\prime}$ be an arbitrary compactification of $Z$. By taking the graph of $f$ : $Z^{\prime} \rightarrow \bar{X}$ and using Hironaka's resolution, we can assume that $Z^{\prime}$ is smooth projective, $\operatorname{Supp}\left(Z^{\prime} \backslash Z\right)$ is simple normal crossing, and $f: Z \rightarrow X$ is extended to $f^{\prime}: Z^{\prime} \rightarrow \bar{X}$. Let $B^{\prime}$ be the closure of $B$ on $Z^{\prime}$. We apply Szabó's resolution lemma (see, for example, $[\mathrm{F} 6])$ to $\operatorname{Supp} B^{\prime} \cup \operatorname{Supp}\left(Z^{\prime} \backslash Z\right)$. Then we obtain the desired variety $\bar{Z}$ and $\bar{B}$. By the above construction, $f$ can be extended to $\bar{f}: \bar{Z} \rightarrow \bar{X}$.

\section{§5. Hodge-theoretic injectivity theorem}

In this section, we will prove the following injectivity theorem, which is a generalization of $[\mathrm{EV}, 5.1$. b)] for $\mathbb{R}$-divisors. We use the classical topology throughout this section.

Proposition 5.1 (Fundamental injectivity theorem). Let $X$ be a smooth projective variety and let $S+B$ be a boundary $\mathbb{R}$-divisor on $X$ such that the support of $S+B$ is simple normal crossing and $\llcorner S+B\lrcorner=S$. Let $L$ be a Cartier divisor on $X$ and let $D$ be an effective Cartier divisor whose support is contained in Supp $B$. Assume that $L \sim_{\mathbb{R}} K_{X}+S+B$. Then the natural homomorphisms

$$
H^{q}\left(X, \mathcal{O}_{X}(L)\right) \rightarrow H^{q}\left(X, \mathcal{O}_{X}(L+D)\right)
$$

which are induced by the natural inclusion $\mathcal{O}_{X} \rightarrow \mathcal{O}_{X}(D)$ are injective for all $q$.

Let us recall some results on the theory of mixed Hodge structures.

5.2 (Mixed Hodge structures). Let $V$ be a smooth projective variety and $\Sigma$ a simple normal crossing divisor on $V$. Let $\iota: V \backslash \Sigma \rightarrow V$ be the natural open immersion. Then $\iota ! \mathbb{C}_{V \backslash \Sigma}$ is quasi-isomorphic to the complex $\Omega_{V}^{\bullet}(\log \Sigma) \otimes \mathcal{O}_{V}(-\Sigma)$. By this quasi-isomorphism, we can construct the spectral sequence

$$
E_{1}^{p q}=H^{q}\left(V, \Omega_{V}^{p}(\log \Sigma) \otimes \mathcal{O}_{V}(-\Sigma)\right) \Rightarrow H_{c}^{p+q}(V \backslash \Sigma, \mathbb{C}) .
$$


By the Serre duality, the left hand side

$$
H^{q}\left(V, \Omega_{V}^{p}(\log \Sigma) \otimes \mathcal{O}_{V}(-\Sigma)\right)
$$

is dual to

$$
H^{n-q}\left(V, \Omega_{V}^{n-p}(\log \Sigma)\right),
$$

where $n=\operatorname{dim} V$. By the Poincaré duality, $H_{c}^{p+q}(V \backslash \Sigma, \mathbb{C})$ is dual to $H^{2 n-(p+q)}(V \backslash \Sigma, \mathbb{C})$. Therefore,

$$
\operatorname{dim} H_{c}^{k}(V \backslash \Sigma, \mathbb{C})=\sum_{p+q=k} \operatorname{dim} H^{q}\left(V, \Omega_{V}^{p}(\log \Sigma) \otimes \mathcal{O}_{V}(-\Sigma)\right)
$$

by Deligne (cf. [D, Corollaire (3.2.13)(ii)]). Thus, the above spectral sequence degenerates at $E_{1}$. We will use this $E_{1}$-degeneration in the proof of Proposition 5.1. By the above $E_{1}$-degeneration, we obtain

$$
H_{c}^{k}(V \backslash \Sigma, \mathbb{C}) \simeq \bigoplus_{p+q=k} H^{q}\left(V, \Omega_{V}^{p}(\log \Sigma) \otimes \mathcal{O}_{V}(-\Sigma)\right) .
$$

In particular, the natural inclusion $\iota ! \mathbb{C}_{V \backslash \Sigma} \subset \mathcal{O}_{V}(-\Sigma)$ induces surjections

$$
H_{c}^{p}(V \backslash \Sigma, \mathbb{C}) \simeq H^{p}\left(V, \iota ! \mathbb{C}_{V \backslash \Sigma}\right) \rightarrow H^{p}\left(V, \mathcal{O}_{V}(-\Sigma)\right)
$$

for all $p$

Proof of Proposition 5.1. By Lemma 4.15, we can assume that $B$ is a $\mathbb{Q}$-divisor and $L \sim_{\mathbb{Q}} K_{X}+S+B$. We put $\mathcal{L}=\mathcal{O}_{X}\left(L-K_{X}-S\right)$. Let $\nu$ be the smallest positive integer such that $\nu L \sim \nu\left(K_{X}+S+B\right)$. In particular, $\nu B$ is an integral Weil divisor. We take the $\nu$-fold cyclic cover $\pi^{\prime}: Y^{\prime}=\operatorname{Spec}_{X} \bigoplus_{i=0}^{\nu-1} \mathcal{L}^{-i} \rightarrow X$ associated to the section $\nu B \in\left|\mathcal{L}^{\nu}\right|$. More precisely, let $s \in H^{0}\left(X, \mathcal{L}^{\nu}\right)$ be a section whose zero divisor is $\nu B$. Then the dual of $s: \mathcal{O}_{X} \rightarrow \mathcal{L}^{\nu}$ defines an $\mathcal{O}_{X}$-algebra structure on $\bigoplus_{i=0}^{\nu-1} \mathcal{L}^{-i}$. Let $Y \rightarrow Y^{\prime}$ be the normalization and let $\pi: Y \rightarrow X$ be the composition morphism. For the details, see [EV, 3.5. Cyclic covers]. We can take a finite cover $\varphi: V \rightarrow Y$ such that $V$ is smooth and $T$ is a simple normal crossing divisor on $V$, where $\psi=\pi \circ \varphi$ and $T=\psi^{*} S$, by Kawamata's covering trick (cf. [EV, 3.17. Lemma]). Let $\iota^{\prime}: Y \backslash \pi^{*} S \rightarrow Y$ be the natural open immersion and let $U$ be the smooth locus of $Y$. We denote the natural open immersion $U \rightarrow Y$ by $j$. We put $\widetilde{\Omega}_{Y}^{p}\left(\log \left(\pi^{*} S\right)\right)=j_{*} \Omega_{U}^{p}\left(\log \left(\pi^{*} S\right)\right)$ for every $p$. Then it can be checked easily that

$$
\iota_{!}^{\prime} \mathbb{C}_{Y \backslash \pi^{*} S} \stackrel{\text { qis }}{\longrightarrow} \widetilde{\Omega}_{Y}^{\bullet}\left(\log \left(\pi^{*} S\right)\right) \otimes \mathcal{O}_{Y}\left(-\pi^{*} S\right)
$$

is a direct summand of

$$
\varphi_{*}\left(\iota ! \mathbb{C}_{V \backslash T}\right) \stackrel{\text { qis }}{\longrightarrow} \varphi_{*}\left(\Omega_{V}^{\bullet}(\log T) \otimes \mathcal{O}_{V}(-T)\right),
$$


where qis means a quasi-isomorphism. On the other hand, we can decompose $\pi_{*}\left(\widetilde{\Omega}_{Y}^{\bullet}\left(\log \left(\pi^{*} S\right)\right) \otimes \mathcal{O}_{Y}\left(-\pi^{*} S\right)\right)$ and $\pi_{*}\left(\iota_{!}^{\prime} \mathbb{C}_{Y \backslash \pi^{*} S}\right)$ into eigencomponents of the Galois action of $\pi: Y \rightarrow X$. We write these decompositions as follows:

$$
\pi_{*}\left(\iota_{!}^{\prime} \mathbb{C}_{Y \backslash \pi^{*} S}\right)=\bigoplus_{i=0}^{\nu-1} \mathcal{C}_{i} \subset \bigoplus_{i=0}^{\nu-1} \mathcal{L}^{-i}(\llcorner i B\lrcorner-S)=\pi_{*} \mathcal{O}_{Y}\left(-\pi^{*} S\right),
$$

where $\mathcal{C}_{i} \subset \mathcal{L}^{-i}(\llcorner i B\lrcorner-S)$ for every $i$. We put $\mathcal{C}=\mathcal{C}_{1}$. We see that

$$
\mathcal{C} \stackrel{\text { qis }}{\longrightarrow} \Omega_{X}^{\bullet}(\log (S+B)) \otimes \mathcal{L}^{-1}(-S)
$$

is a direct summand of

$$
\psi_{*}\left(\iota_{!} \mathbb{C}_{V \backslash T}\right) \stackrel{\text { qis }}{\longrightarrow} \psi_{*}\left(\Omega_{V}^{\bullet}(\log T) \otimes \mathcal{O}_{V}(-T)\right) .
$$

The $E_{1}$-degeneration of the spectral sequence

$$
\begin{aligned}
E_{1}^{p q} & =H^{q}\left(V, \Omega_{V}^{p}(\log T) \otimes \mathcal{O}_{V}(-T)\right) \\
& \Rightarrow \mathbb{H}^{p+q}\left(V, \Omega_{V}^{\bullet}(\log T) \otimes \mathcal{O}_{V}(-T)\right) \simeq H^{p+q}\left(V, \iota_{1} \mathbb{C}_{V \backslash T}\right)
\end{aligned}
$$

(cf. 5.2) implies the $E_{1}$-degeneration of

$$
\begin{aligned}
E_{1}^{p q} & =H^{q}\left(X, \Omega_{X}^{p}(\log (S+B)) \otimes \mathcal{L}^{-1}(-S)\right) \\
& \Rightarrow \mathbb{H}^{p+q}\left(X, \Omega_{X}^{\bullet}(\log (S+B)) \otimes \mathcal{L}^{-1}(-S)\right) \simeq H^{p+q}(X, \mathcal{C}) .
\end{aligned}
$$

Therefore, the inclusion $\mathcal{C} \subset \mathcal{L}^{-1}(-S)$ induces surjections

$$
H^{p}(X, \mathcal{C}) \rightarrow H^{p}\left(X, \mathcal{L}^{-1}(-S)\right)
$$

for all $p$. We can check the following simple property by considering the monodromy action of the Galois group of $\pi: Y \rightarrow X$ on $\mathcal{C}$ around $\operatorname{Supp} B$.

Corollary 5.3 (cf. [KM, Corollary 2.54]). Let $U \subset X$ be a connected open set such that $U \cap \operatorname{Supp} B \neq \emptyset$. Then $H^{0}\left(U,\left.\mathcal{C}\right|_{U}\right)=0$.

This property is utilized via the following fact. The proof is obvious.

Lemma 5.4 (cf. [KM, Lemma 2.55]). Let $F$ be a sheaf of Abelian groups on a topological space $X$ and let $F_{1}, F_{2} \subset F$ be subsheaves. Let $Z \subset X$ be a closed subset. Assume that

(1) $\left.F_{2}\right|_{X \backslash Z}=\left.F\right|_{X \backslash Z}$, and

(2) if $U$ is connected, open and $U \cap Z \neq \emptyset$, then $H^{0}\left(U, F_{1} \mid U\right)=0$.

Then $F_{1}$ is a subsheaf of $F_{2}$. 
As a corollary, we obtain:

Corollary 5.5 (cf. [KM, Corollary 2.56]). Let $M \subset \mathcal{L}^{-1}(-S)$ be a subsheaf such that $\left.M\right|_{X \backslash \operatorname{Supp} B}=\left.\mathcal{L}^{-1}(-S)\right|_{X \backslash \operatorname{Supp} B}$. Then the injection $\mathcal{C} \rightarrow \mathcal{L}^{-1}(-S)$ factors as $\mathcal{C} \rightarrow M \rightarrow \mathcal{L}^{-1}(-S)$. Therefore,

$$
H^{i}(X, M) \rightarrow H^{i}\left(X, \mathcal{L}^{-1}(-S)\right)
$$

is surjective for every $i$.

Proof. The first part is clear from Corollary 5.3 and Lemma 5.4. This implies that we have maps

$$
H^{i}(X, \mathcal{C}) \rightarrow H^{i}(X, M) \rightarrow H^{i}\left(X, \mathcal{L}^{-1}(-S)\right) .
$$

As we saw above, the composition is surjective. Hence so is the right map.

Therefore, we deduce that

$$
H^{q}\left(X, \mathcal{L}^{-1}(-S-D)\right) \rightarrow H^{q}\left(X, \mathcal{L}^{-1}(-S)\right)
$$

is surjective for every $q$. By the Serre duality,

$$
H^{q}\left(X, \mathcal{O}_{X}\left(K_{X}\right) \otimes \mathcal{L}(S)\right) \rightarrow H^{q}\left(X, \mathcal{O}_{X}\left(K_{X}\right) \otimes \mathcal{L}(S+D)\right)
$$

is injective for every $q$. This means that

$$
H^{q}\left(X, \mathcal{O}_{X}(L)\right) \rightarrow H^{q}\left(X, \mathcal{O}_{X}(L+D)\right)
$$

is injective for every $q$.

\section{$\S 6$. Injectivity, torsion-free, and vanishing theorems}

In this section, we prove generalizations of Kollár's torsion-freeness and vanishing theorem (cf. Theorem 6.3). First, we prove a generalization of Kollár's injectivity theorem (cf. [A1, Theorem 3.1]). It is a straightforward consequence of Proposition 5.1 and will produce the desired torsion-free and vanishing theorems.

Theorem 6.1 (Injectivity theorem). Let $X$ be a smooth projective variety and let $\Delta$ be a boundary $\mathbb{R}$-divisor such that $\operatorname{Supp} \Delta$ is simple normal crossing. Let $L$ be a Cartier divisor on $X$ and let $D$ be an effective Cartier divisor that contains no lc centers of $(X, \Delta)$. Assume the following conditions hold:

(i) $L \sim_{\mathbb{R}} K_{X}+\Delta+H$,

(ii) $H$ is a semi-ample $\mathbb{R}$-Cartier $\mathbb{R}$-divisor, and

(iii) $t H \sim_{\mathbb{R}} D+D^{\prime}$ for some positive real number $t$, where $D^{\prime}$ is an effective $\mathbb{R}$-Cartier $\mathbb{R}$-divisor whose support contains no lc centers of $(X, \Delta)$. 
Then the homomorphisms

$$
H^{q}\left(X, \mathcal{O}_{X}(L)\right) \rightarrow H^{q}\left(X, \mathcal{O}_{X}(L+D)\right)
$$

induced by the natural inclusion $\mathcal{O}_{X} \rightarrow \mathcal{O}_{X}(D)$ are injective for all $q$.

Proof. We put $S=\llcorner\Delta\lrcorner$ and $B=\{\Delta\}$. We can take a resolution $f: Y \rightarrow X$ such that $f$ is an isomorphism outside $\operatorname{Supp}\left(D+D^{\prime}+B\right)$, and that the union of the support of $f^{*}\left(S+B+D+D^{\prime}\right)$ and the exceptional locus of $f$ has a simple normal crossing support on $Y$. Let $B^{\prime}$ be the strict transform of $B$ on $Y$. We write

$$
K_{Y}+S^{\prime}+B^{\prime}=f^{*}\left(K_{X}+S+B\right)+E,
$$

where $S^{\prime}$ is the strict transform of $S$ and $E$ is $f$-exceptional. It is easy to see that $E_{+}=\ulcorner E\urcorner \geq 0$. We put $L^{\prime}=f^{*} L+E_{+}$and $E_{-}=E_{+}-E \geq 0$. We note that $E_{+}$ is Cartier and $E_{-}$is an effective $\mathbb{R}$-Cartier $\mathbb{R}$-divisor with $\left\llcorner E_{-}\right\lrcorner=0$. Since $f^{*} H$ is semi-ample, we can write $f^{*} H \sim_{\mathbb{R}} \sum_{i} a_{i} H_{i}^{\prime}$, where $0<a_{i}<1$ and $H_{i}^{\prime}$ is a general Cartier divisor on $Y$ for every $i$. We put

$$
B^{\prime \prime}=B^{\prime}+E_{-}+\frac{\varepsilon}{t} f^{*}\left(D+D^{\prime}\right)+(1-\varepsilon) \sum_{i} a_{i} H_{i}^{\prime}
$$

for some $0<\varepsilon \ll 1$. Then $L^{\prime} \sim_{\mathbb{R}} K_{Y}+S^{\prime}+B^{\prime \prime}$. By the construction, $\left\llcorner B^{\prime \prime}\right\lrcorner=0$, the support of $S^{\prime}+B^{\prime \prime}$ is simple normal crossing on $Y$, and $\operatorname{Supp} B^{\prime \prime} \supset \operatorname{Supp} f^{*} D$. So, Proposition 5.1 implies that the homomorphisms

$$
H^{q}\left(Y, \mathcal{O}_{Y}\left(L^{\prime}\right)\right) \rightarrow H^{q}\left(Y, \mathcal{O}_{Y}\left(L^{\prime}+f^{*} D\right)\right)
$$

are injective for all $q$. It is easy to see that $f_{*} \mathcal{O}_{Y}\left(L^{\prime}\right) \simeq \mathcal{O}_{X}(L)$. By Lemma 4.15, we can write $L^{\prime} \sim_{\mathbb{Q}} K_{Y}+S^{\prime}+B^{\prime \prime \prime}$, where $B^{\prime \prime \prime}$ is a $\mathbb{Q}$-divisor on $Y$ such that $\left\llcorner B^{\prime \prime \prime}\right\lrcorner=0$ and $\operatorname{Supp} B^{\prime \prime \prime}=\operatorname{Supp} B^{\prime \prime}$. Thus, by Lemma 6.2 below, $R^{q} f_{*} \mathcal{O}_{Y}\left(L^{\prime}\right)=0$ for all $q>0$. By the Leray spectral sequence, the homomorphisms

$$
H^{q}\left(X, \mathcal{O}_{X}(L)\right) \rightarrow H^{q}\left(X, \mathcal{O}_{X}(L+D)\right)
$$

are injective for all $q$.

Let us recall the following well-known easy lemma.

Lemma 6.2 (Reid-Fukuda type). Let $V$ be a smooth projective variety and let $B$ be a boundary $\mathbb{Q}$-divisor on $V$ such that $\operatorname{Supp} B$ is simple normal crossing. Let $f: V \rightarrow W$ be a projective birational morphism onto a variety $W$. Assume that $f$ is an isomorphism at the generic point of every lc center of $(V, B)$ and that $D$ is a Cartier divisor on $V$ such that $D-\left(K_{V}+B\right)$ is nef. Then $R^{i} f_{*} \mathcal{O}_{V}(D)=0$ for every $i>0$. 
Proof. We use induction on the number of irreducible components of $\llcorner B\lrcorner$ and on the dimension of $V$. If $\llcorner B\lrcorner=0$, then the lemma follows from the KawamataViehweg vanishing theorem (cf. [KM, Corollary 2.68]). Therefore, we can assume that there is an irreducible divisor $S \subset\llcorner B\lrcorner$. We consider the short exact sequence

$$
0 \rightarrow \mathcal{O}_{V}(D-S) \rightarrow \mathcal{O}_{V}(D) \rightarrow \mathcal{O}_{S}(D) \rightarrow 0
$$

By induction, we see that $R^{i} f_{*} \mathcal{O}_{V}(D-S)=0$ and $R^{i} f_{*} \mathcal{O}_{S}(D)=0$ for every $i>0$. Thus, $R^{i} f_{*} \mathcal{O}_{V}(D)=0$ for $i>0$.

The next theorem is the main theorem of this section (cf. [A1]). See also [F3].

Theorem 6.3 (Torsion-freeness and vanishing theorem). Let $Y$ be a smooth variety and let $B$ be a boundary $\mathbb{R}$-divisor such that $\operatorname{Supp} B$ is simple normal crossing. Let $f: Y \rightarrow X$ be a projective morphism and let $L$ be a Cartier divisor on $Y$ such that $L-\left(K_{Y}+B\right)$ is $f$-semi-ample.

(i) Let $q$ be an arbitrary non-negative integer. Every non-zero local section of $R^{q} f_{*} \mathcal{O}_{Y}(L)$ contains in its support the $f$-image of some stratum of $(Y, B)$.

(ii) Let $\pi: X \rightarrow S$ be a projective morphism. Assume that $L-\left(K_{Y}+B\right) \sim_{\mathbb{R}} f^{*} H$ for some $\pi$-ample $\mathbb{R}$-Cartier $\mathbb{R}$-divisor $H$ on $X$. Then $R^{p} \pi_{*} R^{q} f_{*} \mathcal{O}_{Y}(L)=0$ for every $p>0$ and $q \geq 0$.

Remark 6.4. It is obvious that (i) of Theorem 6.3 is equivalent to

(i') Let $q$ be an arbitrary non-negative integer. Then every associated prime of $R^{q} f_{*} \mathcal{O}_{Y}(L)$ is the generic point of the $f$-image of some stratum of $(Y, B)$.

Proof of Theorem 6.3. We take an $f$-semi-ample $\mathbb{R}$-Cartier $\mathbb{R}$-divisor $M$ on $Y$ such that $M \sim_{\mathbb{R}} L-\left(K_{Y}+B\right)$.

(i) We divide the proof into two steps.

Step 1. First, we assume that $X$ is projective. We can assume that $M$ is semiample by replacing $L$ (resp. $M$ ) with $L+f^{*} A^{\prime}$ (resp. $M+f^{*} A^{\prime}$ ), where $A^{\prime}$ is a very ample Cartier divisor on $X$. Assume that $R^{q} f_{*} \mathcal{O}_{Y}(L)$ has a local section whose support does not contain the images of any $(Y, B)$-strata. More precisely, let $U$ be a non-empty Zariski open set and let $s \in \Gamma\left(U, R^{q} f_{*} \mathcal{O}_{Y}(L)\right)$ be a non-zero section of $R^{q} f_{*} \mathcal{O}_{Y}(L)$ on $U$ whose support $V \subset U$ does not contain the $f$-images of any strata of $(Y, B)$. Let $\bar{V}$ be the closure of $V$ in $X$. We note that $\bar{V} \backslash V$ may contain the $f$-image of some stratum of $(Y, B)$. By replacing $Y$ with its blow-up along an lc center which is mapped into $\bar{V} \backslash V$, we can assume that an irreducible component $B_{0}$ of $\llcorner B\lrcorner$ is mapped into $\bar{V} \backslash V$ by $f$. We note that $M \sim_{\mathbb{R}} L-B_{0}-\left(K_{X}+B-B_{0}\right)$. We replace $L$ (resp. $B$ ) with $L-B_{0}$ (resp. $B-B_{0}$ ). By repeating this process finitely 
many times, we can assume that $\bar{V}$ does not contain the $f$-images of any strata of $(Y, B)$. Then we can find a very ample Cartier divisor $A$ with the following properties:

(a) $f^{*} A$ contains no lc centers of $(Y, B)$, and

(b) $R^{q} f_{*} \mathcal{O}_{Y}(L) \rightarrow R^{q} f_{*} \mathcal{O}_{Y}(L) \otimes \mathcal{O}_{X}(A)$ is not injective.

We can assume that $M-f^{*} A$ is semi-ample by replacing $L$ (resp. $M$ ) with $L+f^{*} A$ (resp. $M+f^{*} A$ ). If necessary, we replace $L$ (resp. $M$ ) with $L+f^{*} A^{\prime \prime}$ (resp. $M+$ $\left.f^{*} A^{\prime \prime}\right)$, where $A^{\prime \prime}$ is a very ample Cartier divisor on $X$. Then

$$
H^{0}\left(X, R^{q} f_{*} \mathcal{O}_{Y}(L)\right) \simeq H^{q}\left(Y, \mathcal{O}_{Y}(L)\right)
$$

and

$$
H^{0}\left(X, R^{q} f_{*} \mathcal{O}_{Y}(L) \otimes \mathcal{O}_{X}(A)\right) \simeq H^{q}\left(Y, \mathcal{O}_{Y}\left(L+f^{*} A\right)\right) .
$$

We see that

$$
H^{0}\left(X, R^{q} f_{*} \mathcal{O}_{Y}(L)\right) \rightarrow H^{0}\left(X, R^{q} f_{*} \mathcal{O}_{Y}(L) \otimes \mathcal{O}_{X}(A)\right)
$$

is not injective by (b) if $A^{\prime \prime}$ is sufficiently ample. So,

$$
H^{q}\left(Y, \mathcal{O}_{Y}(L)\right) \rightarrow H^{q}\left(Y, \mathcal{O}_{Y}\left(L+f^{*} A\right)\right)
$$

is not injective. This contradicts Theorem 6.1.

Step 2. Next, we assume that $X$ is not projective. Note that the problem is local. So, we can shrink $X$ and assume that $X$ is affine. By an argument similar to the one in Step 1 in the proof of (ii) below, we can assume that $M$ is a semi-ample $\mathbb{Q}$-Cartier $\mathbb{Q}$-divisor. We compactify $X$ and apply Lemma 4.17. Then we obtain a compactification $\bar{f}: \bar{Y} \rightarrow \bar{X}$ of $f: Y \rightarrow X$. Let $\bar{M}$ be the closure of $M$ on $\bar{Y}$. If $\bar{M}$ is not a semi-ample $\mathbb{Q}$-Cartier $\mathbb{Q}$-divisor, then we take blowing-ups of $\bar{Y}$ inside $\bar{Y} \backslash Y$ and obtain a semi-ample $\mathbb{Q}$-Cartier $\mathbb{Q}$-divisor $\bar{M}$ on $\bar{Y}$ such that $\left.\bar{M}\right|_{Y}=M$. Let $\bar{L}$ (resp. $\bar{B}$ ) be the closure of $L$ (resp. $B)$ on $\bar{Y}$. We note that $\bar{M} \sim_{\mathbb{R}} \bar{L}-\left(K_{\bar{Y}}+\bar{B}\right)$ does not necessarily hold. We can write $M+\sum_{i} a_{i}\left(f_{i}\right)=L-\left(K_{Y}+B\right)$, where $a_{i}$ is a real number and $f_{i}$ is a rational function on $Y$ for every $i$. We put

$$
E=\bar{M}+\sum_{i} a_{i}\left(f_{i}\right)-\left(\bar{L}-\left(K_{\bar{Y}}+\bar{B}\right)\right) .
$$

We replace $\bar{L}$ (resp. $\bar{B}$ ) with $\bar{L}+\ulcorner E\urcorner$ (resp. $\bar{B}+\{-E\}$ ). Then we obtain the desired property of $R^{q} \bar{f}_{*} \mathcal{O}_{\bar{Y}}(\bar{L})$ since $\bar{X}$ is projective. We note that $\operatorname{Supp} E$ is in $\bar{Y} \backslash Y$. So, this completes the whole proof.

(ii) We divide the proof into three steps. 
Step 1. We assume that $\operatorname{dim} S=0$. The following arguments are well known and standard. We describe them for the reader's convenience. In this case, we can write $H \sim_{\mathbb{R}} H_{1}+H_{2}$, where $H_{1}$ (resp. $H_{2}$ ) is a $\pi$-ample $\mathbb{Q}$-Cartier $\mathbb{Q}$-divisor (resp. $\pi$-ample $\mathbb{R}$-Cartier $\mathbb{R}$-divisor) on $X$. So, we can write $H_{2} \sim_{\mathbb{R}} \sum_{i} a_{i} H_{i}^{\prime}$, where $0<a_{i}<1$ and $H_{i}^{\prime}$ is a general very ample Cartier divisor on $X$ for every $i$. Replacing $B$ (resp. $H$ ) with $B+\sum_{i} a_{i} f^{*} H_{i}^{\prime}$ (resp. $H_{1}$ ), we can assume that $H$ is a $\pi$-ample $\mathbb{Q}$-Cartier $\mathbb{Q}$-divisor. We take a general member $A \in|m H|$, where $m$ is a sufficiently divisible positive integer, such that $A^{\prime}=f^{*} A$ and $R^{q} f_{*} \mathcal{O}_{Y}\left(L+A^{\prime}\right)$ is $\pi_{*}$-acyclic for all $q$. By (i), we have the short exact sequences

$$
0 \rightarrow R^{q} f_{*} \mathcal{O}_{Y}(L) \rightarrow R^{q} f_{*} \mathcal{O}_{Y}\left(L+A^{\prime}\right) \rightarrow R^{q} f_{*} \mathcal{O}_{A^{\prime}}\left(L+A^{\prime}\right) \rightarrow 0
$$

for all $q$. Note that $R^{q} f_{*} \mathcal{O}_{A^{\prime}}\left(L+A^{\prime}\right)$ is $\pi_{*}$-acyclic by induction on $\operatorname{dim} X$ and $R^{q} f_{*} \mathcal{O}_{Y}\left(L+A^{\prime}\right)$ is also $\pi_{*}$-acyclic by the above assumption. Thus, $E_{2}^{p q}=0$ for $p \geq 2$ in the following commutative diagram of spectral sequences:

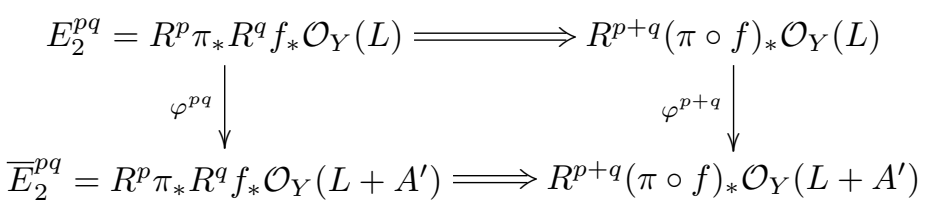

We note that $\varphi^{1+q}$ is injective by Theorem 6.1. Now $E_{2}^{1 q} \rightarrow R^{1+q}(\pi \circ f)_{*} \mathcal{O}_{Y}(L)$ is injective as $E_{2}^{p q}=0$ for $p \geq 2$. Also, $\bar{E}_{2}^{1 q}=0$ by the above assumption. Therefore, $E_{2}^{1 q}=0$ since the injection $E_{2}^{1 q} \rightarrow R^{1+q}(\pi \circ f)_{*} \mathcal{O}_{Y}\left(L+A^{\prime}\right)$ factors through $\bar{E}_{2}^{1 q}=0$. This implies that $R^{p} \pi_{*} R^{q} f_{*} \mathcal{O}_{Y}(L)=0$ for every $p>0$ and $q \geq 0$.

Step 2. We assume that $S$ is projective. By replacing $H$ (resp. $L$ ) with $H+\pi^{*} G$ $\left(\right.$ resp. $\left.L+(\pi \circ f)^{*} G\right)$, where $G$ is a very ample Cartier divisor on $S$, we can assume that $H$ is an ample $\mathbb{R}$-Cartier $\mathbb{R}$-divisor. By the same argument as in Step 1, we can assume that $H$ is an ample $\mathbb{Q}$-Cartier $\mathbb{Q}$-divisor and $M \sim \mathbb{Q} f^{*} H$. If $G$ is a sufficiently ample Cartier divisor on $S, H^{k}\left(S, R^{p} \pi_{*} R^{q} f_{*} \mathcal{O}_{Y}(L) \otimes \mathcal{O}_{S}(G)\right)=0$ for every $k \geq 1$,

$$
\begin{aligned}
H^{0}\left(S, R^{p} \pi_{*} R^{q} f_{*} \mathcal{O}_{Y}(L) \otimes \mathcal{O}_{S}(G)\right) & \simeq H^{p}\left(X, R^{q} f_{*} \mathcal{O}_{Y}(L) \otimes \mathcal{O}_{X}\left(\pi^{*} G\right)\right) \\
& \simeq H^{p}\left(X, R^{q} f_{*} \mathcal{O}_{Y}\left(L+f^{*} \pi^{*} G\right)\right),
\end{aligned}
$$

and $R^{p} \pi_{*} R^{q} f_{*} \mathcal{O}_{Y}(L) \otimes \mathcal{O}_{S}(G)$ is generated by its global sections. Since

$$
M+f^{*} \pi^{*} G \sim_{\mathbb{R}} L+f^{*} \pi^{*} G-\left(K_{Y}+B\right), \quad M+f^{*} \pi^{*} G \sim_{\mathbb{Q}} f^{*}\left(H+\pi^{*} G\right),
$$

and $H+\pi^{*} G$ is ample, we can apply Step 1 to obtain $H^{p}\left(X, R^{q} f_{*} \mathcal{O}_{Y}\left(L+f^{*} \pi^{*} G\right)\right)$ $=0$ for every $p>0$ and $q \geq 0$. Thus, $R^{p} \pi_{*} R^{q} f_{*} \mathcal{O}_{Y}(L)=0$ for every $p>0$ and $q \geq 0$ by the above arguments. 
Step 3. When $S$ is not projective, we shrink $S$ and assume that $S$ is affine. By the same argument as in Step 1 above, we can assume that $H$ is $\mathbb{Q}$-Cartier. We compactify $S$ and $X$, and can assume that $S$ and $X$ are projective. By Lemma 4.17 , we can reduce it to the case when $S$ is projective. This step is essentially the same as Step 2 in the proof of (i). So, we omit the details here.

We have thus obtained the statement (ii).

\section{$\S 7$. Non-lc ideal sheaves}

We introduce the notion of non-lc ideal sheaves. It is an analogue of the usual multiplier ideal sheaves (see, for example, [L, Chapter 9]). For details, see [F10] and [FST].

Definition 7.1 (Non-lc ideal sheaf). Let $X$ be a normal variety and let $B$ be an $\mathbb{R}$-divisor on $X$ such that $K_{X}+B$ is $\mathbb{R}$-Cartier. Let $f: Y \rightarrow X$ be a resolution with $K_{Y}+B_{Y}=f^{*}\left(K_{X}+B\right)$ such that Supp $B_{Y}$ is simple normal crossing. Then we put

$$
\mathcal{J}_{\mathrm{NLC}}(X, B)=f_{*} \mathcal{O}_{Y}\left(\left\ulcorner-\left(B_{Y}^{<1}\right)\right\urcorner-\left\llcorner B_{Y}^{>1}\right\lrcorner\right)=f_{*} \mathcal{O}_{Y}\left(-\left\llcorner B_{Y}\right\lrcorner+B_{Y}^{=1}\right)
$$

and call it the non-lc ideal sheaf associated to $(X, B)$. If $B$ is effective, then $\mathcal{J}_{\mathrm{NLC}}(X, B) \subset \mathcal{O}_{X}$.

The ideal sheaf $\mathcal{J}_{\mathrm{NLC}}(X, B)$ is well defined by the following easy lemma.

Lemma 7.2. Let $g: Z \rightarrow Y$ be a proper birational morphism between smooth varieties and let $B_{Y}$ be an $\mathbb{R}$-divisor on $Y$ such that Supp $B_{Y}$ is simple normal crossing. Assume that $K_{Z}+B_{Z}=g^{*}\left(K_{Y}+B_{Y}\right)$ and that $\operatorname{Supp} B_{Z}$ is simple normal crossing. Then

$$
g_{*} \mathcal{O}_{Z}\left(\left\ulcorner-\left(B_{Z}^{<1}\right)\right\urcorner-\left\llcorner B_{Z}^{>1}\right\lrcorner\right) \simeq \mathcal{O}_{Y}\left(\left\ulcorner-\left(B_{Y}^{<1}\right)\right\urcorner-\left\llcorner B_{Y}^{>1}\right\lrcorner\right) .
$$

Proof. Since $K_{Z}+B_{Z}=g^{*}\left(K_{Y}+B_{Y}\right)$, we obtain

$$
\begin{aligned}
K_{Z}= & g^{*}\left(K_{Y}+B_{Y}^{=1}+\left\{B_{Y}\right\}\right) \\
& +g^{*}\left(\left\llcorner B_{Y}^{<1}\right\lrcorner+\left\llcorner B_{Y}^{>1}\right\lrcorner\right)-\left(\left\llcorner B_{Z}^{<1}\right\lrcorner+\left\llcorner B_{Z}^{>1}\right\lrcorner\right)-B_{Z}^{=1}-\left\{B_{Z}\right\} .
\end{aligned}
$$

If $a\left(\nu, Y, B_{\bar{Y}}^{=1}+\left\{B_{Y}\right\}\right)=-1$ for a prime divisor $\nu$ over $Y$, then we can check that $a\left(\nu, Y, B_{Y}\right)=-1$ by using [KM, Lemma 2.45]. Since $g^{*}\left(\left\llcorner B_{Y}^{<1}\right\lrcorner+\left\llcorner B_{Y}^{>1}\right\lrcorner\right)-$ $\left(\left\llcorner B_{Z}^{<1}\right\lrcorner+\left\llcorner B_{Z}^{>1}\right\lrcorner\right)$ is Cartier, we can easily see that

$$
g^{*}\left(\left\llcorner B_{Y}^{<1}\right\lrcorner+\left\llcorner B_{Y}^{>1}\right\lrcorner\right)=\left\llcorner B_{Z}^{<1}\right\lrcorner+\left\llcorner B_{Z}^{>1}\right\lrcorner+E,
$$


where $E$ is an effective $f$-exceptional Cartier divisor. Thus, we obtain

$$
g_{*} \mathcal{O}_{Z}\left(\left\ulcorner-\left(B_{Z}^{<1}\right)\right\urcorner-\left\llcorner B_{Z}^{>1}\right\lrcorner\right) \simeq \mathcal{O}_{Y}\left(\left\ulcorner-\left(B_{Y}^{<1}\right)\right\urcorner-\left\llcorner B_{Y}^{>1}\right\lrcorner\right) .
$$

This completes the proof.

Remark 7.3. We use the same notation as in Definition 7.1. We put

$$
\mathcal{J}(X, B)=f_{*} \mathcal{O}_{Y}\left(-\left\llcorner B_{Y}\right\lrcorner\right) .
$$

This sheaf $\mathcal{J}(X, B)$ is well known as the (algebraic version of) multiplier ideal sheaf of the pair $(X, B)$. See, for example, [L, Chapter 9].

By the definition, the following proposition is obvious.

Proposition 7.4. Let $X$ be a normal variety and let $B$ be an effective $\mathbb{R}$-divisor on $X$ such that $K_{X}+B$ is $\mathbb{R}$-Cartier. Then $(X, B)$ is log canonical if and only if $\mathcal{J}_{\mathrm{NLC}}(X, B)=\mathcal{O}_{X}$.

The next proposition is a kind of Bertini's theorem.

Proposition 7.5. Let $X$ be a smooth variety and let $B$ be an effective $\mathbb{R}$-divisor on $X$ such that $K_{X}+B$ is $\mathbb{R}$-Cartier. Let $\Lambda$ be a linear system on $X$ and let $D \in \Lambda$ be a general member of $\Lambda$. Then

$$
\mathcal{J}_{\mathrm{NLC}}(X, B+t D)=\mathcal{J}_{\mathrm{NLC}}(X, B)
$$

outside the base locus $\operatorname{Bs} \Lambda$ of $\Lambda$ for all $0 \leq t \leq 1$.

Proof. By replacing $X$ with $X \backslash \operatorname{Bs} \Lambda$, we can assume that Bs $\Lambda=\emptyset$. Let $f: Y \rightarrow X$ be a resolution as in Definition 7.1. Since $D$ is a general member of $\Lambda, f^{*} D=f_{*}^{-1} D$ is a smooth divisor on $Y$ such that $\operatorname{Supp} f^{*} D \cup \operatorname{Supp} B_{Y}$ is simple normal crossing. Hence, we can check that $\mathcal{J}_{\mathrm{NLC}}(X, B+t D)=\mathcal{J}_{\mathrm{NLC}}(X, B)$ for all $0 \leq t \leq 1$.

We close this section with an important remark.

Remark 7.6. In the subsequent sections (Sections 8, 12, 13, and 15), we consider the scheme structure of $\operatorname{Nlc}(X, B)$ defined by $\mathcal{J}_{\mathrm{NLC}}(X, B)$. However, we can use $\mathcal{J}^{\prime}(X, B)$ or $\mathcal{J}_{l}^{\prime}(X, B)$ for any negative integer $l$ in place of $\mathcal{J}_{\mathrm{NLC}}(X, B)$. For the definitions and basic properties of $\mathcal{J}^{\prime}(X, B)$ and $\mathcal{J}_{l}^{\prime}(X, B)$, see [FST]. We adopt $\mathcal{J}_{\text {NLC }}(X, B)$ since we think it is the most natural defining ideal sheaf of $\operatorname{Nlc}(X, B)$.

\section{$\S 8$. Vanishing theorem}

The following vanishing theorem, which is a special case of [A1, Theorem 4.4], is one of the key results in this paper. We note that the importance of Theorem 8.1 is in its formulation best suited for new frameworks explained in subsequent 
sections. For the details of Ambro's original statement, see [A1, Theorem 4.4] or [F11, Theorem 3.39].

Theorem 8.1. Let $X$ be a normal variety and let $B$ be an effective $\mathbb{R}$-divisor on $X$ such that $K_{X}+B$ is $\mathbb{R}$-Cartier. Let $D$ be a Cartier divisor on $X$. Assume that $D-\left(K_{X}+B\right)$ is $\pi$-ample, where $\pi: X \rightarrow S$ is a projective morphism onto a variety $S$. Let $\left\{C_{i}\right\}$ be any set of lc centers of the pair $(X, B)$. Put $W=\bigcup C_{i}$ with the reduced scheme structure. Assume that $W$ is disjoint from $\operatorname{Nlc}(X, B)$. Then

$$
R^{i} \pi_{*}\left(\mathcal{J} \otimes \mathcal{O}_{X}(D)\right)=0 \quad \text { for every } i>0,
$$

where $\mathcal{J}=\mathcal{I}_{W} \cdot \mathcal{J}_{\mathrm{NLC}}(X, B) \subset \mathcal{O}_{X}$ and $\mathcal{I}_{W}$ is the defining ideal sheaf of $W$ on $X$. Therefore, the restriction map

$$
\pi_{*} \mathcal{O}_{X}(D) \rightarrow \pi_{*} \mathcal{O}_{W}(D) \oplus \pi_{*} \mathcal{O}_{\mathrm{Nlc}(X, B)}(D)
$$

is surjective and

$$
R^{i} \pi_{*} \mathcal{O}_{W}(D)=0 \quad \text { for every } i>0 .
$$

In particular, the restriction maps

$$
\pi_{*} \mathcal{O}_{X}(D) \rightarrow \pi_{*} \mathcal{O}_{W}(D) \quad \text { and } \quad \pi_{*} \mathcal{O}_{X}(D) \rightarrow \pi_{*} \mathcal{O}_{\mathrm{Nlc}(X, B)}(D)
$$

are surjective.

Proof. Let $f: Y \rightarrow X$ be a resolution such that $\operatorname{Supp} f_{*}^{-1} B \cup \operatorname{Exc}(f)$ is a simple normal crossing divisor. We can further assume that $f^{-1}(W)$ is a simple normal crossing divisor on $Y$. We can write

$$
K_{Y}+B_{Y}=f^{*}\left(K_{X}+B\right) .
$$

Let $T$ be the union of the irreducible components of $B_{Y}^{=1}$ that are mapped into $W$ by $f$. We consider the short exact sequence

$$
0 \rightarrow \mathcal{O}_{Y}(A-N-T) \rightarrow \mathcal{O}_{Y}(A-N) \rightarrow \mathcal{O}_{T}(A-N) \rightarrow 0,
$$

where $A=\left\ulcorner-\left(B_{Y}^{<1}\right)\right\urcorner$ and $N=\left\llcorner B_{Y}^{>1}\right\lrcorner$. Note that $A$ is an effective $f$-exceptional divisor. We obtain the long exact sequence

$$
\begin{aligned}
0 \rightarrow f_{*} \mathcal{O}_{Y}(A-N-T) \rightarrow f_{*} \mathcal{O}_{Y}(A-N) & \rightarrow f_{*} \mathcal{O}_{T}(A-N) \\
& \stackrel{\delta}{\rightarrow} R^{1} f_{*} \mathcal{O}_{Y}(A-N-T) \rightarrow \cdots .
\end{aligned}
$$

Since

$$
A-N-T-\left(K_{Y}+\left\{B_{Y}\right\}+B_{Y}^{=1}-T\right)=-\left(K_{Y}+B_{Y}\right) \sim_{\mathbb{R}}-f^{*}\left(K_{X}+B\right),
$$


every non-zero local section of $R^{1} f_{*} \mathcal{O}_{Y}(A-N-T)$ contains in its support the $f$-image of some stratum of $\left(Y,\left\{B_{Y}\right\}+B_{Y}^{=1}-T\right)$ by Theorem 6.3(i). On the other hand, $W=f(T)$. Therefore, the connecting homomorphism $\delta$ is a zero map. Thus, we have a short exact sequence

$$
0 \rightarrow f_{*} \mathcal{O}_{Y}(A-N-T) \rightarrow f_{*} \mathcal{O}_{Y}(A-N) \rightarrow f_{*} \mathcal{O}_{T}(A-N) \rightarrow 0 .
$$

We put $\mathcal{J}=f_{*} \mathcal{O}_{Y}(A-N-T) \subset \mathcal{O}_{X}$. Since $W$ is disjoint from $\operatorname{Nlc}(X, B)$, the ideal sheaf $\mathcal{J}$ coincides with $\mathcal{I}_{W}$ (resp. $\mathcal{J}_{\mathrm{NLC}}(X, B)$ ) in a neighborhood of $W$ (resp. $\operatorname{Nlc}(X, B)$ ). Therefore, $\mathcal{J}=\mathcal{I}_{W} \cdot \mathcal{J}_{\mathrm{NLC}}(X, B)$. We put $U=X \backslash \operatorname{Nlc}(X, B)$ and $V=f^{-1}(U)$. By restricting $(\diamond)$ to $U$, we obtain

$$
0 \rightarrow f_{*} \mathcal{O}_{V}(A-T) \rightarrow f_{*} \mathcal{O}_{V}(A) \rightarrow f_{*} \mathcal{O}_{T}(A) \rightarrow 0 .
$$

Since $f_{*} \mathcal{O}_{V}(A) \simeq \mathcal{O}_{U}$, we have $f_{*} \mathcal{O}_{T}(A) \simeq \mathcal{O}_{W}$. The latter isomorphism plays a crucial role in the next section, so we write it as a proposition.

Proposition 8.2. We have $f_{*} \mathcal{O}_{T}(A) \simeq \mathcal{O}_{W}$. This obviously implies that $f_{*} \mathcal{O}_{T}$ $\simeq \mathcal{O}_{W}$ since $A$ is effective.

Remark 8.3. We did not use $D$ or $\pi: X \rightarrow S$ to obtain Proposition 8.2.

Since

$$
f^{*} D+A-N-T-\left(K_{Y}+\left\{B_{Y}\right\}+B_{Y}^{=1}-T\right) \sim_{\mathbb{R}} f^{*}\left(D-\left(K_{X}+B\right)\right),
$$

we have

$$
R^{i} \pi_{*}\left(\mathcal{J} \otimes \mathcal{O}_{X}(D)\right) \simeq R^{i} \pi_{*}\left(f_{*} \mathcal{O}_{Y}(A-N-T) \otimes \mathcal{O}_{X}(D)\right)=0
$$

for every $i>0$ by Theorem 6.3(ii). By considering the short exact sequence

$$
0 \rightarrow \mathcal{J} \rightarrow \mathcal{J}_{\mathrm{NLC}}(X, B) \rightarrow \mathcal{O}_{W} \rightarrow 0,
$$

we obtain

$$
\begin{aligned}
\cdots \rightarrow R^{i} \pi_{*}\left(\mathcal{J}_{\mathrm{NLC}}(X, B)\right. & \left.\otimes \mathcal{O}_{X}(D)\right) \\
& \rightarrow R^{i} \pi_{*} \mathcal{O}_{W}(D) \rightarrow R^{i+1} \pi_{*}\left(\mathcal{J} \otimes \mathcal{O}_{X}(D)\right) \rightarrow \cdots .
\end{aligned}
$$

Since we have already checked

$$
R^{i} \pi_{*}\left(\mathcal{J}_{\mathrm{NLC}}(X, B) \otimes \mathcal{O}_{X}(D)\right)=R^{i} \pi_{*}\left(\mathcal{J} \otimes \mathcal{O}_{X}(D)\right)=0
$$

for every $i>0$, we have $R^{i} \pi_{*} \mathcal{O}_{W}(D)=0$ for all $i>0$. Finally, we consider the short exact sequence

$$
0 \rightarrow \mathcal{J} \rightarrow \mathcal{O}_{X} \rightarrow \mathcal{O}_{W} \oplus \mathcal{O}_{\mathrm{Nlc}(X, B)} \rightarrow 0 .
$$


By taking $\otimes \mathcal{O}_{X}(D)$ and $R^{i} \pi_{*}$, we obtain

$$
0 \rightarrow \pi_{*}\left(\mathcal{J} \otimes \mathcal{O}_{X}(D)\right) \rightarrow \pi_{*} \mathcal{O}_{X}(D) \rightarrow \pi_{*} \mathcal{O}_{W}(D) \oplus \pi_{*} \mathcal{O}_{\mathrm{Nlc}}(X, B)(D) \rightarrow 0
$$

This completes the proof of Theorem 8.1.

\section{$\S 9$. Lc centers}

We prove the basic properties of lc centers as an application of the result in the preceding section (cf. Proposition 8.2). Theorem 9.1 is very useful in the study of linear systems on log canonical pairs. It cannot be proved by the traditional method based on the Kawamata-Viehweg-Nadel vanishing theorem in the sense that the coefficients of $B$ cannot be perturbed in general. For related topics, see [FG].

Theorem 9.1 (cf. [A1, Propositions 4.7 and 4.8]). Let $X$ be a normal variety and let $B$ be an effective $\mathbb{R}$-divisor such that $(X, B)$ is log canonical. Then we have the following properties:

(1) $(X, B)$ has at most finitely many lc centers.

(2) The intersection of two lc centers is a union of lc centers.

(3) Any union of lc centers of $(X, B)$ is semi-normal.

(4) Let $x \in X$ be a closed point such that $(X, B)$ is lc but not klt at $x$. Then there is a unique minimal lc center $W_{x}$ passing through $x$, and $W_{x}$ is normal at $x$.

Proof. We use the notation in the proof of Theorem 8.1. (1) is obvious. (3) is also obvious by Proposition 8.2 since $T$ is a simple normal crossing divisor. Let $C_{1}$ and $C_{2}$ be two lc centers of $(X, B)$. We fix a closed point $P \in C_{1} \cap C_{2}$. For the proof of (2), it is enough to find an lc center $C$ such that $P \in C \subset C_{1} \cap C_{2}$. We put $W=C_{1} \cup C_{2}$. By Proposition 8.2, we obtain $f_{*} \mathcal{O}_{T} \simeq \mathcal{O}_{W}$. This means that $f: T \rightarrow W$ has connected fibers. We note that $T$ is a simple normal crossing divisor on $Y$. Thus, there exist irreducible components $T_{1}$ and $T_{2}$ of $T$ such that $T_{1} \cap T_{2} \cap f^{-1}(P) \neq \emptyset$ and $f\left(T_{i}\right) \subset C_{i}$ for $i=1,2$. Therefore, we can find an lc center $C$ with $P \in C \subset C_{1} \cap C_{2}$. This yields (2).

Finally, we prove (4). The existence and uniqueness of the minimal lc center follow from (2). We take the unique minimal lc center $W=W_{x}$ passing through $x$. By Proposition 8.2, we have $f_{*} \mathcal{O}_{T} \simeq \mathcal{O}_{W}$. By shrinking $W$ around $x$, we can assume that every stratum of $T$ dominates $W$. Thus, $f: T \rightarrow W$ factors through the normalization $W^{\nu}$ of $W$. Since $f_{*} \mathcal{O}_{T} \simeq \mathcal{O}_{W}$, we see that $W^{\nu} \rightarrow W$ is an isomorphism. So, we obtain (4). 


\section{$\S 10$. Dlt blow-ups}

In this section, we discuss dlt blow-ups by Hacon (cf. Theorem 10.4). In the subsequent sections, we will only use Lemma 10.2 (well known to experts) and Theorem 10.4. For details, see Sections 11 and 18. We also discuss a slight refinement of dlt blow-ups (cf. Theorem 10.5), which is useful for future studies of $\log$ canonical pairs and has already played a crucial role in the study of log canonical weak Fano pairs (cf. [G]).

Let us recall the definition of dlt pairs. For another definition and the basic properties of dlt pairs, see [KM, Section 2.3] and [F6].

Definition 10.1 (Dlt pair). Let $X$ be a normal variety and let $B$ be an effective $\mathbb{R}$-divisor on $X$ such that $K_{X}+B$ is $\mathbb{R}$-Cartier. If there exists a resolution $f$ : $Y \rightarrow X$ such that

(i) both $\operatorname{Exc}(f)$ and $\operatorname{Exc}(f) \cup \operatorname{Supp} f_{*}^{-1} B$ are simple normal crossing divisors on $Y$, and

(ii) $a(E, X, B)>-1$ for every exceptional divisor $E \subset Y$,

then $(X, B)$ is called divisorial log terminal ( $d l t$, for short).

We will use the following lemma in Section 11. For the details, see [F6, 3.9 Adjunction for dlt pairs].

Lemma 10.2. Let $(X, B)$ be a dlt pair and let $V$ be an lc center of $(X, B)$. Then $K_{V}+B_{V}=\left.\left(K_{X}+B\right)\right|_{V}$ is dlt by adjunction.

We borrow the next theorem from [BCHM].

Theorem 10.3 (cf. [BCHM, Theorem 1.2]). Let $(X, B)$ be a klt pair, where $K_{X}+B$ is $\mathbb{R}$-Cartier. Let $\pi: X \rightarrow S$ be a projective birational morphism of quasiprojective varieties. Then $(X, B)$ has a log terminal model over $S$. This means that there exists a projective birational morphism $f: X^{\prime} \rightarrow S$ such that

(i) $X^{\prime}$ is $\mathbb{Q}$-factorial,

(ii) $\phi^{-1}$ has no exceptional divisors, where $\phi=f^{-1} \circ \pi: X \rightarrow X^{\prime}$,

(iii) $K_{X^{\prime}}+B^{\prime}$ is $f$-nef, where $B^{\prime}=\phi_{*} B$, and

(iv) $a(E, X, B)<a\left(E, X^{\prime}, B^{\prime}\right)$ for every $\phi$-exceptional divisor $E \subset X$.

The following theorem is very useful. The proof, which is the same as that of [KK, Theorem 3.1], is nevertheless given below since Theorem 10.5 is based on the close examination of it and Section 6. For a simpler proof of Theorem 10.4, see [F18, Section 4]. 
Theorem 10.4 (Dlt blow-ups by Hacon, cf. [KK, Theorem 3.1]). Let $X$ be $a$ normal quasi-projective variety and let $B$ be a boundary $\mathbb{R}$-divisor on $X$ such that $K_{X}+B$ is $\mathbb{R}$-Cartier. In this case, we can construct a projective birational morphism $f: Y \rightarrow X$ from a normal quasi-projective variety $Y$ with the following properties:

(i) $Y$ is $\mathbb{Q}$-factorial.

(ii) $a(E, X, B) \leq-1$ for every $f$-exceptional divisor $E$ on $Y$.

(iii) Put

$$
B_{Y}=f_{*}^{-1} B+\sum_{E: f \text {-exceptional }} E .
$$

Then $\left(Y, B_{Y}\right)$ is dlt and

$$
K_{Y}+B_{Y}=f^{*}\left(K_{X}+B\right)+\sum_{a(E, X, B)<-1}(a(E, X, B)+1) E .
$$

In particular, if $(X, B)$ is lc, then $K_{Y}+B_{Y}=f^{*}\left(K_{X}+B\right)$. Moreover, if $(X, B)$ is dlt, then we can make $f$ small, that is, $f$ is an isomorphism in codimension one.

Proof. Let $\pi: V \rightarrow X$ be a resolution such that $\pi_{*}^{-1} B \cup \operatorname{Exc}(\pi)$ has a simple normal crossing support. We can assume that $\pi$ is a composite of blow-ups of centers of codimension at least two. Then there exists an effective $\pi$-exceptional Cartier divisor $C$ on $V$ such that $-C$ is $\pi$-ample. We put

$$
F=\sum_{\substack{a(E, X, B)>-1, E: \pi \text {-exceptional }}} E \text { and } E^{+}=-\sum_{a(E, X, B) \leq-1} a(E, X, B) E .
$$

We note that $E^{+}$is not necessarily $\pi$-exceptional. We put $E=\operatorname{Supp} E^{+}$. We note that $E^{+}-E$ is $\pi$-exceptional.

Let $H$ be a sufficiently ample Cartier divisor on $X$. We choose $0<\epsilon, \nu, \mu \ll 1$ and note that

$$
\text { (ด) } \begin{aligned}
E+(1-\nu) F+\mu(-C & \left.+\pi^{*} H\right) \\
& =(1-\epsilon \mu) E+(1-\nu) F+\mu\left(\epsilon E-C+\pi^{*} H\right) .
\end{aligned}
$$

Since $-C+\pi^{*} H$ and $\epsilon E-C+\pi^{*} H$ are ample, we can take effective $\mathbb{Q}$-divisors $H_{1}$ and $H_{2}$ with small coefficients such that $E+F+\pi^{*} B+H_{1}+H_{2}$ has a simple normal crossing support and that $-C+\pi^{*} H \sim_{\mathbb{Q}} H_{1}, \epsilon E-C+\pi^{*} H \sim_{\mathbb{Q}} H_{2}$. Then $\left(V,(1-\epsilon \mu) E+(1-\nu) F+\pi_{*}^{-1} B^{<1}+\mu H_{2}\right)$ is klt. By Theorem 10.3, it has a log terminal model $f: Y \rightarrow X$. By [BCHM], we can assume that $\varphi: V \rightarrow Y$ is a composition of $\left(K_{V}+(1-\epsilon \mu) E+(1-\nu) F+\pi_{*}^{-1} B^{<1}+\mu H_{2}\right)$-negative divisorial 
contractions and log flips. By equation $(\mathbf{A})$, this is also a relative minimal model of the pair $\left(V, E+(1-\nu) F+\pi_{*}^{-1} B^{<1}+\mu H_{1}\right)$, which is therefore dlt.

For any divisor $G$ on $V$ appearing above, let $G^{\prime}$ denote its transform on $Y$. By the above construction,

$$
N=K_{Y}+(1-\epsilon \mu) E^{\prime}+(1-\nu) F^{\prime}+f_{*}^{-1} B^{<1}+\mu H_{2}^{\prime}
$$

is $f$-nef and $K_{Y}+\bar{B}=f^{*}\left(K_{X}+B\right)$ is $\mathbb{R}$-linearly $f$-trivial. We put

$$
D=\bar{B}-E^{\prime}-(1-\nu) F^{\prime}-f_{*}^{-1} B^{<1}+\mu C^{\prime} .
$$

Then

$$
-D \sim_{\mathbb{R}, f} N-\left(K_{Y}+\bar{B}\right)=-\bar{B}+(1-\epsilon \mu) E^{\prime}+(1-\nu) F^{\prime}+f_{*}^{-1} B^{<1}+\mu H_{2}^{\prime},
$$

hence it is $f$-nef. Since $f_{*} D=0$, we see that $D$ is effective by the negativity lemma (cf. Lemma 4.16).

Every divisor in $F$ has a negative coefficient in

$$
\widetilde{B}-E-(1-\nu) F-\pi_{*}^{-1} B^{<1}+\mu C,
$$

where $K_{V}+\widetilde{B}=\pi^{*}\left(K_{X}+B\right)$. Therefore, $F$ is contracted on $Y$. So, every $f$ exceptional divisor has discrepancy $\leq-1$. By the above construction, $\left(Y, E^{\prime}+\right.$ $\left.f_{*}^{-1} B^{<1}+\mu H_{1}^{\prime}\right)$ is dlt since $F^{\prime}=0$. Therefore, $\left(Y, E^{\prime}+f_{*}^{-1} B^{<1}\right)$ is also dlt. This means that $\left(Y, B_{Y}\right)$ is dlt because $B_{Y}=E^{\prime}+\sum f_{*}^{-1} B^{<1}$.

When $(X, B)$ is dlt, we can assume that $E^{+}=\pi_{*}^{-1} B^{=1}$ by the definition of dlt pairs. Therefore, we can make $f$ small.

The study of lc centers of a given lc pair often requires a refinement of Theorem 10.4, that is, Theorem 10.5 which relates lc centers on $X$ to those on $Y$. It is actually needed in [G], which studies weak Fano varieties with log canonical singularities.

Theorem 10.5. Let $X$ be a normal quasi-projective variety and let $B$ be an effective $\mathbb{R}$-divisor on $X$ such that $(X, B)$ is lc. In this case, we can construct a projective birational morphism $f: Y \rightarrow X$ from a normal quasi-projective variety $Y$ with the following properties:

(i) $Y$ is $\mathbb{Q}$-factorial.

(ii) $a(E, X, B)=-1$ for every $f$-exceptional divisor $E$ on $Y$.

(iii) Put

$$
B_{Y}=f_{*}^{-1} B+\sum_{E: f \text {-exceptional }} E .
$$

Then $\left(Y, B_{Y}\right)$ is dlt and $K_{Y}+B_{Y}=f^{*}\left(K_{X}+B\right)$. 
(iv) Let $\left\{C_{i}\right\}$ be any set of lc centers of $(X, B)$. Put $W=\bigcup C_{i}$ with the reduced scheme structure. Let $S$ be the union of the irreducible components of $B_{Y}^{=1}$ which are mapped into $W$ by $f$. Then $f_{*} \mathcal{O}_{S} \simeq \mathcal{O}_{W}$.

Proof. Let $\pi: V \rightarrow X$ be a resolution such that

(1) $\pi^{-1}(C)$ is a simple normal crossing divisor on $V$ for every lc center $C$ of $(X, B)$, and

(2) $\pi_{*}^{-1} B \cup \operatorname{Exc}(\pi) \cup \pi^{-1}(\operatorname{Nklt}(X, B))$ has a simple normal crossing support.

We apply the arguments in the proof of Theorem 10.4. From now on, we use the same notation as in the proof of Theorem 10.4. In this case, we have

$$
E=\operatorname{Supp} E^{+}=E^{+} \text {. }
$$

When we construct $f: Y \rightarrow X$, we can run the log minimal model program with scaling with respect to

$K_{V}+E+(1-\nu) F+\pi_{*}^{-1} B^{<1}+\mu H_{1} \sim_{\mathbb{R}} K_{V}+(1-\varepsilon \mu) E+(1-\nu) F+\pi_{*}^{-1} B^{<1}+\mu H_{2}$

(cf. $[\mathrm{BCHM}])$. So, we can assume that $\varphi: V \rightarrow Y$ is a composition of $\left(K_{V}+\right.$ $\left.E+(1-\nu) F+\pi_{*}^{-1} B^{<1}+\mu H_{1}\right)$-negative divisorial contractions and log flips. Let $\Sigma$ be an lc center of $\left(Y, B_{Y}\right)$. Then it is also an lc center of $\left(Y, B_{Y}+\mu H_{1}^{\prime}\right)$. By the negativity lemma (cf. Lemma 4.16), $\varphi: V \rightarrow Y$ is an isomorphism around the generic point of $\Sigma$. Therefore, if $f(\Sigma) \subset W$, then $\Sigma \subset S$ by the conditions (1) and (2) for $\pi: V \rightarrow X$. This means that no lc centers of $\left(Y, B_{Y}-S\right)$ are mapped into $W$ by $f$. Let $g: Z \rightarrow Y$ be a resolution such that

(a) $K_{Z}+B_{Z}=g^{*}\left(K_{Y}+B_{Y}\right)$,

(b) Supp $B_{Z}$ is a simple normal crossing divisor, and

(c) $g$ is an isomorphism over the generic point of any lc center of $\left(Y, B_{Y}\right)$.

Let $S_{Z}$ be the strict transform of $S$ on $Z$. We consider the short exact sequence

$$
0 \rightarrow \mathcal{O}_{Z}\left(\left\ulcorner-\left(B_{Z}^{<1}\right)\right\urcorner-S_{Z}\right) \rightarrow \mathcal{O}_{Z}\left(\left\ulcorner-\left(B_{Z}^{<1}\right)\right\urcorner\right) \rightarrow \mathcal{O}_{S_{Z}}\left(\left\ulcorner-\left(B_{Z}^{<1}\right)\right\urcorner\right) \rightarrow 0 .
$$

We note that

$$
\left\ulcorner-\left(B_{Z}^{<1}\right)\right\urcorner-S_{Z}-\left(K_{Z}+\left\{B_{Z}\right\}+B_{Z}^{=1}-S_{Z}\right) \sim_{\mathbb{R}}-h^{*}\left(K_{X}+B\right),
$$

where $h=f \circ g$. Then we obtain

$$
\begin{array}{r}
0 \rightarrow h_{*} \mathcal{O}_{Z}\left(\left\ulcorner-\left(B_{Z}^{<1}\right)\right\urcorner-S_{Z}\right) \rightarrow h_{*} \mathcal{O}_{Z}\left(\left\ulcorner-\left(B_{Z}^{<1}\right)\right\urcorner\right) \rightarrow h_{*} \mathcal{O}_{S_{Z}}\left(\left\ulcorner-\left(B_{Z}^{<1}\right)\right\urcorner\right) \\
\stackrel{\delta}{\rightarrow} R^{1} h_{*} \mathcal{O}_{Z}\left(\left\ulcorner-\left(B_{Z}^{<1}\right)\right\urcorner-S_{Z}\right) \rightarrow \cdots .
\end{array}
$$


Every associated prime of $R^{1} h_{*} \mathcal{O}_{Z}\left(\left\ulcorner-\left(B_{Z}^{<1}\right)\right\urcorner-S_{Z}\right)$ is the generic point of the $h$-image of some stratum of $\left(Z,\left\{B_{Z}\right\}+B_{Z}^{-1}-S_{Z}\right)$ by Theorem 6.3(i) and no lc centers of $\left(Z,\left\{B_{Z}\right\}+B_{\bar{Z}}^{\overline{1}}-S_{Z}\right)$ are mapped into $W$ by $h$. Therefore, $\delta$ is a zero map. Thus, we obtain

$$
0 \rightarrow \mathcal{I}_{W} \rightarrow \mathcal{O}_{X} \rightarrow h_{*} \mathcal{O}_{S_{Z}}\left(\left\ulcorner-\left(B_{Z}^{<1}\right)\right\urcorner\right) \rightarrow 0
$$

and $\mathcal{O}_{W} \simeq h_{*} \mathcal{O}_{S_{Z}} \simeq h_{*} \mathcal{O}_{S_{Z}}\left(\left\ulcorner-\left(B_{Z}^{<1}\right)\right\urcorner\right)\left(\right.$ cf. Proposition 8.2), where $\mathcal{I}_{W}$ is the defining ideal sheaf of $W$. Here, we have used the fact that $\left\ulcorner-\left(B_{Z}^{<1}\right)\right\urcorner$ is effective and $h$-exceptional. By applying $g_{*}$ to $(\nabla)$, we obtain

$$
0 \rightarrow \mathcal{I}_{S} \rightarrow \mathcal{O}_{Y} \rightarrow g_{*} \mathcal{O}_{S_{Z}}\left(\left\ulcorner-\left(B_{Z}^{<1}\right)\right\urcorner\right) \rightarrow 0
$$

and $\mathcal{O}_{S} \simeq g_{*} \mathcal{O}_{S_{Z}} \simeq g_{*} \mathcal{O}_{S_{Z}}\left(\left\ulcorner-\left(B_{Z}^{<1}\right)\right\urcorner\right)\left(\right.$ cf. Proposition 8.2), where $\mathcal{I}_{S} \simeq \mathcal{O}_{Y}(-S)$ is the defining ideal sheaf of $S$. We note that

$$
R^{1} g_{*} \mathcal{O}_{Z}\left(\left\ulcorner-\left(B_{Z}^{<1}\right)\right\urcorner-S_{Z}\right)=0
$$

by Theorem $6.3(\mathrm{i})$ since $g$ is an isomorphism at the generic point of any stratum of $\left(Z,\left\{B_{Z}\right\}+B_{Z}^{=1}-S_{Z}\right)$, and $\left\ulcorner-\left(B_{Z}^{<1}\right)\right\urcorner$ is effective and $g$-exceptional. Therefore, $\mathcal{O}_{W} \simeq h_{*} \mathcal{O}_{S_{Z}} \simeq f_{*} g_{*} \mathcal{O}_{S_{Z}} \simeq f_{*} \mathcal{O}_{S}$

\section{§11. Vanishing theorem for minimal lc centers}

In this section, we prove a vanishing theorem on minimal lc centers. It is very powerful and will play a crucial role in the proof of Theorem 12.1. We note that a key point of Theorem 11.1 is in its formulation which is best suited for our subsequent applications.

Theorem 11.1 (Vanishing theorem for minimal lc centers). Let $X$ be a normal variety and let $B$ be an effective $\mathbb{R}$-divisor on $X$ such that $K_{X}+B$ is $\mathbb{R}$-Cartier. Let $W$ be a minimal lc center of $(X, B)$ such that $W$ is disjoint from $\operatorname{Nlc}(X, B)$. Let $\pi: X \rightarrow S$ be a projective morphism onto a variety $S$. Let $D$ be a Cartier divisor on $W$ such that $D-\left.\left(K_{X}+B\right)\right|_{W}$ is $\pi$-ample. Then

$$
R^{i} \pi_{*} \mathcal{O}_{W}(D)=0 \quad \text { for every } i>0 .
$$

Proof. Without loss of generality, we can assume that $S$ is quasi-projective. We shrink $X$ around $W$ and assume that $(X, B)$ is $\log$ canonical. By Theorem 10.4, we can construct a projective birational morphism $f: Y \rightarrow X$ such that $K_{Y}+B_{Y}=$ $f^{*}\left(K_{X}+B\right)$ and $\left(Y, B_{Y}\right)$ is dlt. We take an lc center $V$ of $\left(Y, B_{Y}\right)$ such that $f(V)=W$ and put $K_{V}+B_{V}=\left.\left(K_{Y}+B_{Y}\right)\right|_{V}$. Then $\left(V, B_{V}\right)$ is dlt by Lemma 10.2 and $K_{V}+B_{V} \sim_{\mathbb{R}} f^{*}\left(\left.\left(K_{X}+B\right)\right|_{W}\right)$. Let $g: Z \rightarrow V$ be a resolution such that 
$K_{Z}+B_{Z}=g^{*}\left(K_{V}+B_{V}\right)$ and Supp $B_{Z}$ is simple normal crossing. Then we have $K_{Z}+B_{Z} \sim_{\mathbb{R}} h^{*}\left(\left.\left(K_{X}+B\right)\right|_{W}\right)$, where $h=f \circ g$. Since

$$
h^{*}\left(D-\left.\left(K_{X}+B\right)\right|_{W}\right) \sim_{\mathbb{R}} h^{*} D+\left\ulcorner-\left(B_{Z}^{<1}\right)\right\urcorner-\left(K_{Z}+B_{Z}^{=1}+\left\{B_{Z}\right\}\right),
$$

we obtain

$$
R^{i} \pi_{*} h_{*} \mathcal{O}_{Z}\left(h^{*} D+\left\ulcorner-\left(B_{Z}^{<1}\right)\right\urcorner\right)=0 \quad \text { for every } i>0
$$

by Theorem $6.3($ ii). We note that

$$
h_{*} \mathcal{O}_{Z}\left(h^{*} D+\left\ulcorner-\left(B_{Z}^{<1}\right)\right\urcorner\right) \simeq f_{*} \mathcal{O}_{V}\left(f^{*} D\right)
$$

by the projection formula since $\left\ulcorner-\left(B_{Z}^{<1}\right)\right\urcorner$ is effective and $g$-exceptional. Moreover, $\mathcal{O}_{W}(D)$ is a direct summand of $f_{*} \mathcal{O}_{V}\left(f^{*} D\right) \simeq \mathcal{O}_{W}(D) \otimes f_{*} \mathcal{O}_{V}$ since $W$ is normal (cf. Theorem 9.1(4)). Therefore, $R^{i} \pi_{*} \mathcal{O}_{W}(D)=0$ for every $i>0$.

We close this section with an important remark.

Remark 11.2. The short proof of Theorem 11.1 given in this section depends on Theorem 6.3(ii), Theorem 9.1(4), and Theorem 10.4 which is a corollary to [BCHM]. However Theorem 11.1, a special case of [A1, Theorem 4.4], is independent of $[\mathrm{BCHM}]$ since it can be proved without using Theorem 10.4. We refer the reader to [F11, Theorem 3.39] for the independent proof of Theorem 11.1, which heavily depends on the theory of mixed Hodge structures on compact support cohomology groups of reducible varieties (cf. [F11, Chapter 2]).

\section{$\S 12$. Non-vanishing theorem}

In this section, we prove the non-vanishing theorem, which is a generalization of the main theorem of [F16]. In [A1], Ambro does not discuss any generalization of Shokurov's non-vanishing theorem. Therefore, the result in this section is one of the main differences between the theory of quasi-log varieties and our new framework.

Theorem 12.1 (Non-vanishing theorem). Let $X$ be a normal variety and let $B$ be an effective $\mathbb{R}$-divisor on $X$ such that $K_{X}+B$ is $\mathbb{R}$-Cartier. Let $\pi: X \rightarrow S$ be a projective morphism onto a variety $S$ and let $L$ be a $\pi$-nef Cartier divisor on $X$. Assume that

(i) $a L-\left(K_{X}+B\right)$ is $\pi$-ample for some real number $a>0$, and

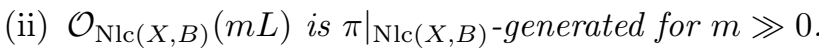

Then the relative base locus $\mathrm{Bs}_{\pi}|m L|$ contains no lc centers of $(X, B)$ and is disjoint from $\operatorname{Nlc}(X, B)$ for $m \gg 0$. 
Proof. Without loss of generality, we can assume that $S$ is affine.

Step 1. In this step, we will prove that $\mathcal{O}_{X}(m L)$ is $\pi$-generated on an open neighborhood of $\operatorname{Nlc}(X, B)$ for $m \gg 0$.

By the assumption, $\pi^{*} \pi_{*} \mathcal{O}_{\mathrm{Nlc}(X, B)}(m L) \rightarrow \mathcal{O}_{\mathrm{Nlc}(X, B)}(m L)$ is surjective for $m \gg 0$. On the other hand, $\pi_{*} \mathcal{O}_{X}(m L) \rightarrow \pi_{*} \mathcal{O}_{\mathrm{Nlc}(X, B)}(m L)$ is surjective for $m \geq a$ since

$$
R^{1} \pi_{*}\left(\mathcal{J}_{\mathrm{NLC}}(X, B) \otimes \mathcal{O}_{X}(m L)\right)=0
$$

for $m \geq a$ by Theorem 8.1. Therefore, for every large integer $m, \pi^{*} \pi_{*} \mathcal{O}_{X}(m L) \rightarrow$ $\mathcal{O}_{X}(m L)$ is surjective on an open neighborhood of $\operatorname{Nlc}(X, B)$. See the following commutative diagram:

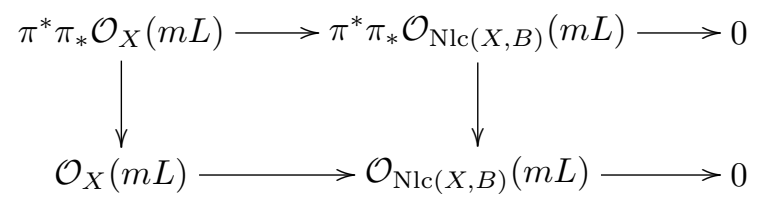

Let $W$ be a minimal lc center of $(X, B)$. Then it is sufficient to see that $W$ is not contained in $\operatorname{Bs}|m L|$ for $m \gg 0$.

Step 2. If $W \cap \operatorname{Nlc}(X, B) \neq \emptyset$, then Bs $|m L|$ does not contain $W$ by Step 1. So, from now on, we can assume that $W \cap \operatorname{Nlc}(X, B)=\emptyset$.

Step 3. We assume that $\left.L\right|_{W_{\eta}}$ is numerically trivial, where $W_{\eta}$ is the generic fiber of $W \rightarrow \pi(W)$. In this case,

$$
h^{0}\left(W_{\eta}, \mathcal{O}_{W_{\eta}}(L)\right)=\chi\left(W_{\eta}, \mathcal{O}_{W_{\eta}}(L)\right)=\chi\left(W_{\eta}, \mathcal{O}_{W_{\eta}}\right)=h^{0}\left(W_{\eta}, \mathcal{O}_{W_{\eta}}\right)>0
$$

by [Kl, Chapter, II $\S 2$, Theorem 1] and the vanishing theorem (Theorem 11.1). On the other hand,

$$
\pi_{*} \mathcal{O}_{X}(m L) \rightarrow \pi_{*} \mathcal{O}_{W}(m L) \oplus \pi_{*} \mathcal{O}_{\mathrm{Nlc}(X, B)}(m L)
$$

is surjective for every $m \geq a$ by Theorem 8.1. In particular, the restriction map $\pi_{*} \mathcal{O}_{X}(m L) \rightarrow \pi_{*} \mathcal{O}_{W}(m L)$ is surjective for every $m \geq a$. Thus, Bs $|m L|$ does not contain $W$ for every $m \geq a$.

Step 4. We assume that $\left.L\right|_{W_{\eta}}$ is not numerically trivial. We take a general subvariety $V$ of $W$ such that $V \rightarrow \pi(W)$ is generically finite. If $l$ is a large positive integer, then we can write

$$
l L-\left(K_{X}+B\right)=N_{1}+a_{2} N_{2}+\cdots+a_{k} N_{k}
$$

with the following properties: 
(a) $N_{1}$ is a $\pi$-ample $\mathbb{Q}$-Cartier $\mathbb{Q}$-divisor on $X$ such that

$$
\left(\left.\left(\left.N_{1}\right|_{W}\right)\right|_{F}\right)^{\operatorname{dim} F}>d\left(\operatorname{codim}_{W} V\right)^{\operatorname{dim} F},
$$

where $d$ is the mapping degree of $V \rightarrow \pi(W)$ and $F$ is a general fiber of $W \rightarrow \pi(W)$.

(b) $a_{i}$ is a positive real number and $N_{i}$ is a $\pi$-very ample Cartier divisor on $X$ for every $i \geq 2$.

By Lemma 12.2 below, we can find an effective $\mathbb{Q}$-divisor $D_{1}$ on $W$ such that $\left.D_{1} \sim_{\mathbb{Q}} N_{1}\right|_{W}$ with mult $D_{V}>\operatorname{codim}_{W} V$. If $b$ is sufficiently large and divisible, then $\left.b D_{1} \sim b N_{1}\right|_{W}, \mathcal{I}_{W} \otimes \mathcal{O}_{X}\left(b N_{1}\right)$ is $\pi$-generated, and $R^{1} \pi_{*}\left(\mathcal{I}_{W} \otimes \mathcal{O}_{X}\left(b N_{1}\right)\right)=0$ since $N_{1}$ is $\pi$-ample, where $\mathcal{I}_{W}$ is the defining ideal sheaf of $W$. By using the short exact sequence

$$
0 \rightarrow \pi_{*}\left(\mathcal{I}_{W} \otimes \mathcal{O}_{X}\left(b N_{1}\right)\right) \rightarrow \pi_{*} \mathcal{O}_{X}\left(b N_{1}\right) \rightarrow \pi_{*} \mathcal{O}_{W}\left(b N_{1}\right) \rightarrow 0,
$$

we can find an effective $\mathbb{Q}$-divisor $M_{1}$ on $X$ with the following properties:

(a) $\left.M_{1}\right|_{W}=D_{1}$.

(b) $M_{1} \sim_{\mathbb{Q}} N_{1}$.

(c) $\left(X, B+M_{1}\right)$ is lc outside $W \cup \operatorname{Nlc}(X, B)$.

(d) $\mathcal{J}_{\mathrm{NLC}}\left(X, B+M_{1}\right)=\mathcal{J}_{\mathrm{NLC}}(X, B)$ outside $W$

Let $M_{i}$ be a general member of $\left|N_{i}\right|$ for every $i \geq 2$. We put $M=M_{1}+a_{2} M_{2}+$ $\cdots+a_{k} M_{k}$. Then we have:

(i) $\left.M\right|_{W} \geq D_{1}$.

(ii) $M \sim_{\mathbb{R}} l L-\left(K_{X}+B\right)$.

(iii) $(X, B+M)$ is lc outside $W \cup \operatorname{Nlc}(X, B)$.

(iv) $\mathcal{J}_{\mathrm{NLC}}(X, B+M)=\mathcal{J}_{\mathrm{NLC}}(X, B)$ outside $W$

We take the $\log$ canonical threshold $c$ of $(X, B)$ with respect to $M$ outside $\operatorname{Nlc}(X, B)$. By the above construction, we have $0<c<1$. More precisely, $c>0$ since $M$ contains no lc centers of $(X, B)$, and $c<1$ follows from the fact that $\left.M\right|_{W} \geq D_{1}$ and $\operatorname{mult}_{V} D_{1}>\operatorname{codim}_{W} V$. We note that

$$
(a-a c+c l) L-\left(K_{X}+B+c M\right) \sim_{\mathbb{R}}(1-c)\left(a L-\left(K_{X}+B\right)\right)
$$

is $\pi$-ample. Moreover, we can find a smaller lc center $W^{\prime}$ of $(X, B+c M)$ contained in $W$ (cf. Theorem 9.1(2)). Therefore, we replace $(X, B)$ with $(X, B+c M), a$ with $a-a c+c l$, and consider the new lc center $W^{\prime}$. By repeating this process, we reach the situation where $\left.L\right|_{W_{\eta}}$ is numerically trivial. 
Anyway, we have proved that Bs $|m L|$ contains no lc centers of $(X, B)$ for $m \gg 0$.

The following lemma is a relative version of Shokurov's concentration method. We used it in the proof of Theorem 12.1.

Lemma 12.2. Let $f: Y \rightarrow Z$ be a projective morphism from a normal variety $Y$ onto an affine variety $Z$. Let $V$ be a general closed subvariety of $Y$ such that $f: V \rightarrow Z$ is generically finite. Let $M$ be an $f$-ample $\mathbb{R}$-divisor on $Y$. Assume that

$$
\left(\left.M\right|_{F}\right)^{d}>k m^{d}
$$

where $F$ is a general fiber of $f: Y \rightarrow Z, d=\operatorname{dim} F$, and $k$ is the mapping degree of $f: V \rightarrow Z$. Then we can find an effective $\mathbb{R}$-divisor $D$ on $Y$ such that

$$
D \sim_{\mathbb{R}} M
$$

and mult $_{V} D>m$. If $M$ is a $\mathbb{Q}$-divisor, then we can make $D$ a $\mathbb{Q}$-divisor with $D \sim_{\mathbb{Q}} M$.

Proof. We can write

$$
M=M_{1}+a_{2} M_{2}+\cdots+a_{l} M_{l},
$$

where $M_{1}$ is an $f$-ample $\mathbb{Q}$-Cartier $\mathbb{Q}$-divisor such that $\left(\left.M_{1}\right|_{F}\right)^{d}>k m^{d}, a_{i}$ is a positive real number, and $M_{i}$ is an $f$-ample Cartier divisor for every $i$. If $M$ is a $\mathbb{Q}$-divisor, then we can assume that $l=2$ and $a_{2}$ is rational. Let $\mathcal{I}_{V}$ be the defining ideal sheaf of $V$ on $Y$. We consider the exact sequence

$$
0 \rightarrow f_{*}\left(\mathcal{O}_{Y}\left(p M_{1}\right) \otimes \mathcal{I}_{V}^{p m}\right) \rightarrow f_{*} \mathcal{O}_{Y}\left(p M_{1}\right) \rightarrow f_{*}\left(\mathcal{O}_{Y}\left(p M_{1}\right) \otimes \mathcal{O}_{Y} / \mathcal{I}_{V}^{p m}\right) \rightarrow \cdots
$$

for a sufficiently large and divisible integer $p$. By restricting the above sequence to a general fiber $F$ of $f$, we can check that the rank of $f_{*} \mathcal{O}_{Y}\left(p M_{1}\right)$ is greater than that of $f_{*}\left(\mathcal{O}_{Y}\left(p M_{1}\right) \otimes \mathcal{O}_{Y} / \mathcal{I}_{V}^{p m}\right)$ by the usual estimates (see Lemma 12.3 below). Therefore, $f_{*}\left(\mathcal{O}_{Y}\left(p M_{1}\right) \otimes \mathcal{I}_{V}^{p m}\right) \neq 0$. Let $D_{1}$ be a member of

$$
H^{0}\left(Z, f_{*}\left(\mathcal{O}_{Y}\left(p M_{1}\right) \otimes \mathcal{I}_{V}^{p m}\right)\right)=H^{0}\left(Y, \mathcal{O}_{Y}\left(p M_{1}\right) \otimes \mathcal{I}_{V}^{p m}\right)
$$

and let $D_{i}$ be an effective $\mathbb{Q}$-Cartier $\mathbb{Q}$-divisor such that $D_{i} \sim_{\mathbb{Q}} M_{i}$ for $i \geq 2$. We can take $D_{2}$ with mult ${ }_{V} D_{2}>0$. Then $D=(1 / p) D_{1}+a_{2} D_{2}+\cdots+a_{l} D_{l}$ has the desired properties.

We close this section with the following well-known lemma. The proof is obvious. 
Lemma 12.3. Let $X$ be a normal projective variety with $\operatorname{dim} X=d$ and let $A$ be an ample $\mathbb{Q}$-divisor on $X$ such that $r A$ is Cartier for some positive integer $r$. Then

$$
h^{0}\left(X, \mathcal{O}_{X}(\operatorname{tr} A)\right)=\chi\left(X, \mathcal{O}_{X}(\operatorname{tr} A)\right)=\frac{(\operatorname{tr} A)^{d}}{d !}+(\text { lower terms in } t)
$$

by the Riemann-Roch formula and the Serre vanishing theorem for $t \gg 0$.

Let $P \in X$ be a smooth point. Then

$$
\operatorname{dim}_{\mathbb{C}} \mathcal{O}_{X} / m_{P}^{\alpha}=\left(\begin{array}{c}
\alpha-1+d \\
d
\end{array}\right)=\frac{\alpha^{d}}{d !}+(\text { lower terms in } \alpha)
$$

for all $\alpha \geq 1$, where $m_{P}$ is the maximal ideal associated to $P$.

\section{$\S 13$. Base point free theorem}

The base point free theorem is one of the most important theorems in the log minimal model program. Since we have already established the non-vanishing theorem (Theorem 12.1) in our framework, there are no difficulties in obtaining the base point free theorem (Theorem 13.1). Our approach is simpler than in [A1], though Theorem 13.1 is a special case of the base point free theorem for quasi-log varieties obtained by Ambro (cf. [A1, Theorem 5.1] and [F11, Theorem 3.66]). Indeed in the approach of [A1] it is necessary to treat reducible non-equidimensional quasi-log varieties even for the proof of the base point free theorem for log canonical pairs.

Theorem 13.1 (Base point free theorem). Let $X$ be a normal variety and let $B$ be an effective $\mathbb{R}$-divisor on $X$ such that $K_{X}+B$ is $\mathbb{R}$-Cartier. Let $\pi: X \rightarrow S$ be a projective morphism onto a variety $S$ and let $L$ be a $\pi$-nef Cartier divisor on $X$. Assume that

(i) $a L-\left(K_{X}+B\right)$ is $\pi$-ample for some real number $a>0$, and

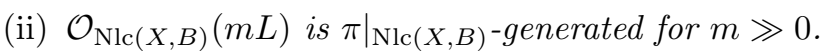

Then $\mathcal{O}_{X}(m L)$ is $\pi$-generated for $m \gg 0$.

We will prove the base point free theorem for $\mathbb{R}$-divisors in Section 17 as an application of the cone theorem (Theorem 16.6).

Proof. We can assume that $S$ is affine.

Step 1. We assume that $(X, B)$ is klt and that $L_{\eta}$ is numerically trivial, where $L_{\eta}=\left.L\right|_{X_{\eta}}$ and $X_{\eta}$ is the generic fiber of $\pi: X \rightarrow S$. Then we have

$$
h^{0}\left(X_{\eta}, \mathcal{O}_{X_{\eta}}\left(L_{\eta}\right)\right)=\chi\left(X_{\eta}, \mathcal{O}_{X_{\eta}}\left(L_{\eta}\right)\right)=\chi\left(X_{\eta}, \mathcal{O}_{X_{\eta}}\right)=h^{0}\left(X_{\eta}, \mathcal{O}_{X_{\eta}}\right)>0
$$


by $[\mathrm{Kl}$, Chapter II, $\S 2$, Theorem 1] and the vanishing theorem. Here, the Kawamata -Viehweg vanishing theorem is sufficient. Therefore, $|L| \neq \emptyset$. Let $D$ be a member of $|L|$. If $D=0$, then it is obvious that $|m L|$ is free for every $m$. Thus, we can assume that $D \neq 0$. Let $c$ be the $\log$ canonical threshold of $(X, B)$ with respect to $D$. We replace $(X, B)$ with $(X, B+c D)$, and $a$ with $a+c$. Then we can assume that $(X, B)$ is lc but not klt. This case will be treated in Step 3 .

Step 2. We assume that $(X, B)$ is klt and that $L_{\eta}$ is not numerically trivial. We take a general subvariety $V$ on $X$ such that $\pi: V \rightarrow S$ is generically finite. By Lemma 12.2 , we can find an effective $\mathbb{R}$-divisor $D$ on $X$ such that

$$
D \sim_{\mathbb{R}} l L-\left(K_{X}+B\right)
$$

for some large $l$ and $\operatorname{mult}_{V} D>\operatorname{codim}_{X} V$. Let $c$ be the log canonical threshold of $(X, B)$ with respect to $D$. By the above construction, we obtain $0<c<1$. We replace $(X, B)$ with $(X, B+c D)$, and $a$ with $a-a c+c l$, and can assume that $(X, B)$ is lc but not klt. We note that

$$
(a-a c+c l) L-\left(K_{X}+B+c D\right) \sim_{\mathbb{R}}(1-c)\left(a L-\left(K_{X}+B\right)\right) .
$$

So, the problem is reduced to the case when $(X, B)$ is lc but not klt. It will be treated in Step 3.

Step 3. We assume that $(X, B)$ is not klt. Let $p$ be a prime integer. We will prove that $\operatorname{Bs}\left|p^{m} L\right|=\emptyset$ for some positive integer $m$.

By Theorem 12.1, $\left|p^{m_{1}} L\right| \neq \emptyset$ for some positive integer $m_{1}$. If $\operatorname{Bs}\left|p^{m_{1}} L\right|=\emptyset$, then there is nothing to prove. So, we can assume that $\operatorname{Bs}\left|p^{m_{1}} L\right| \neq \emptyset$. We take general members $D_{1}, \ldots, D_{n+1} \in\left|p^{m_{1}} L\right|$, where $n=\operatorname{dim} X$. Since $D_{1}, \ldots, D_{n+1}$ are general, $\left(X, B+D_{1}+\cdots+D_{n+1}\right)$ is lc outside Bs $\left|p^{m_{1}} L\right| \cup \operatorname{Nlc}(X, B)$. It is easy to see that $(X, B+D)$, where $D=D_{1}+\cdots+D_{n+1}$, is not lc at the generic point of every irreducible component of $\operatorname{Bs}\left|p^{m_{1}} L\right|$ (see Lemma 13.2 below). Let $c$ be the $\log$ canonical threshold of $(X, B)$ with respect to $D$ outside $\operatorname{Nlc}(X, B)$. Then $(X, B+c D)$ is lc but not klt outside $\operatorname{Nlc}(X, B), 0<c<1$, and $\mathcal{J}_{\mathrm{NLC}}(X, B+c D)=$ $\mathcal{J}_{\mathrm{NLC}}(X, B)$ (see Proposition 7.5). We note that

$$
\left(c(n+1) p^{m_{1}}+a\right) L-\left(K_{X}+B+c D\right) \sim_{\mathbb{R}} a L-\left(K_{X}+B\right)
$$

is $f$-ample. By the construction, there exists an lc center of $(X, B+c D)$ contained in $\operatorname{Bs}\left|p^{m_{1}} L\right|$. By Theorem 12.1, we can find $m_{2}>m_{1}$ such that $\operatorname{Bs}\left|p^{m_{2}} L\right| \subsetneq$ $\operatorname{Bs}\left|p^{m_{1}} L\right|$. By noetherian induction, there exists $m$ such that $\operatorname{Bs}\left|p^{m} L\right|=\emptyset$. 
Step 4. Let $p^{\prime}$ be a prime integer such that $p^{\prime} \neq p$. Then, by Step 3 again, we can find a positive integer $m^{\prime}$ such that $\operatorname{Bs}\left|p^{\prime m^{\prime}} L\right|=\emptyset$. So, there exists a positive integer $m_{0}$ such that $|k L|$ is free for every $k \geq m_{0}$, since $\operatorname{Bs}\left|p^{m} L\right|=\emptyset$ and $\operatorname{Bs}\left|p^{\prime m^{\prime}} L\right|=\emptyset$.

This completes the proof.

We close this section with the following lemma. We used it in the proof of Theorem 13.1.

Lemma 13.2. Let $X$ be a normal variety and let $B$ be an effective $\mathbb{R}$-divisor on $X$ such that $K_{X}+B$ is $\mathbb{R}$-Cartier. Let $P$ be a closed point of $X$ and let $P \in D_{i}$ be a Cartier divisor for every $i$. If $\left(X, B+\sum_{i=1}^{k} D_{i}\right)$ is $\log$ canonical at $P$, then $k \leq \operatorname{dim} X$.

Proof. The proof is by induction on $\operatorname{dim} X$. The assertion is clear if $\operatorname{dim} X=1$. We put $S=D_{1}$. Let $\nu: S^{\nu} \rightarrow S$ be the normalization and let $B_{S^{\nu}}$ be the different of $(X, S+B)$ on $S^{\nu}$ (see Section 14 below). So, we have $K_{S^{\nu}}+B_{S^{\nu}}=\nu^{*}\left(K_{X}+S+B\right)$. Since $\left(X, B+S+\sum_{i=2}^{k} D_{i}\right)$ is log canonical at $P,\left(S^{\nu}, B_{S^{\nu}}+\sum_{i=2}^{k} \nu^{*} D_{i}\right)$ is $\log$ canonical at $Q \in \nu^{-1}(P)$. Thus, $k-1 \leq \operatorname{dim} S^{\nu}$ by induction. This means that $k \leq \operatorname{dim} X$.

\section{§14. Shokurov's differents}

Let us recall the definition and basic properties of Shokurov's differents following $[\mathrm{S} 2, \S 3]$ and $[\mathrm{A} 2,9.2 .1]$.

14.1. Let $X$ be a normal variety and let $S+B$ be an $\mathbb{R}$-divisor on $X$ such that $K_{X}+S+B$ is $\mathbb{R}$-Cartier. Assume that $S$ is reduced and that $S$ and $B$ have no common irreducible components. Let $f: Y \rightarrow X$ be a resolution such that

$$
K_{Y}+S_{Y}+B_{Y}=f^{*}\left(K_{X}+S+B\right)
$$

and $\operatorname{Supp}\left(S_{Y}+B_{Y}\right)$ is simple normal crossing and $S_{Y}$ is smooth, where $S_{Y}$ is the strict transform of $S$ on $Y$. Let $\nu: S^{\nu} \rightarrow S$ be the normalization. Then $f: S_{Y} \rightarrow S$ can be decomposed as

$$
f: S_{Y} \stackrel{\pi}{\longrightarrow} S^{\nu} \stackrel{\nu}{\longrightarrow} S .
$$

We define $B_{S_{Y}}=\left.B_{Y}\right|_{S_{Y}}$. Then we obtain

$$
\left.\left(K_{Y}+S_{Y}+B_{Y}\right)\right|_{S_{Y}}=K_{S_{Y}}+B_{S_{Y}}
$$

by adjunction. We put $B_{S^{\nu}}=\pi_{*} B_{S_{Y}}$. Then we have

$$
K_{S^{\nu}}+B_{S^{\nu}}=\nu^{*}\left(K_{X}+S+B\right) .
$$


The $\mathbb{R}$-divisor $B_{S^{\nu}}$ on $S^{\nu}$ is called the different of $(X, S+B)$ on $S^{\nu}$. We can easily check that $B_{S^{\nu}}$ is independent of the resolution $f: Y \rightarrow X$. So, $B_{S^{\nu}}$ is a well-defined $\mathbb{R}$-divisor on $S^{\nu}$. We can check the following properties:

(i) $K_{S^{\nu}}+B_{S^{\nu}}$ is $\mathbb{R}$-Cartier and $K_{S^{\nu}}+B_{S^{\nu}}=\nu^{*}\left(K_{X}+S+B\right)$.

(ii) If $B$ is a $\mathbb{Q}$-divisor, then so is $B_{S^{\nu}}$.

(iii) $B_{S^{\nu}}$ is effective if $B$ is effective in a neighborhood of $S$.

(iv) $\left(S^{\nu}, B_{S^{\nu}}\right)$ is $\log$ canonical if $(X, S+B)$ is $\log$ canonical in a neighborhood of $S$.

(v) Let $D$ be an $\mathbb{R}$-Cartier $\mathbb{R}$-divisor on $X$ such that $S$ and $D$ have no common irreducible components. Then

$$
(B+D)_{S^{\nu}}=B_{S^{\nu}}+\nu^{*} D .
$$

We sometimes write $\left.D\right|_{S^{\nu}}=\nu^{*} D$ for simplicity.

The above properties except (iii) are obvious by the definition. We give a proof of (iii) for the reader's convenience.

Proof of (iii). By shrinking $X$, we can assume that $X$ is quasi-projective and $B$ is effective. By taking hyperplane cuts, we can also assume that $X$ is a surface. Run the log minimal model program over $X$ with respect to $K_{Y}+S_{Y}$. Let $C$ be a curve on $Y$ such that $\left(K_{Y}+S_{Y}\right) \cdot C<0$ and $f(C)$ is a point. Then $K_{Y} \cdot C<0$ because $S_{Y}$ is the strict transform of $S$. Therefore, each step of the log minimal model program over $X$ with respect to $K_{Y}+S_{Y}$ is a contraction of a $(-1)$-curve $E$ with $\left(K_{Y}+S_{Y}\right) \cdot E<0$. So, by replacing $\left(Y, S_{Y}\right)$ with the output of the above log minimal model program, we can assume that $Y$ is smooth, $\left(Y, S_{Y}\right)$ is plt, and $K_{Y}+S_{Y}$ is $f$-nef. We note that $S_{Y}$ is a smooth curve since $\left(Y, S_{Y}\right)$ is plt (cf. [KM, Proposition 5.51]). By the negativity lemma (see Lemma 4.16) and the assumption that $B$ is effective, $B_{Y}$ is effective. We note the equality

$$
-B_{Y}=K_{Y}+S_{Y}-f^{*}\left(K_{X}+S+B\right) .
$$

By adjunction, we obtain

$$
\left.\left(K_{Y}+S_{Y}+B_{Y}\right)\right|_{S_{Y}}=K_{S_{Y}}+\left.B_{Y}\right|_{S_{Y}} .
$$

Obviously, $\left.B_{Y}\right|_{S_{Y}}$ is effective. This implies that $B_{S^{\nu}}=\left.B_{Y}\right|_{S_{Y}}$ is effective.

When $X$ is singular, $B_{S^{\nu}}$ is not necessarily zero even if $B=0$. 


\section{$\S 15$. Rationality theorem}

In this section, we prove the rationality theorem below, though it is a special case of [A1, Theorem 5.9] (see also [F11, Theorem 3.68]). In the traditional Xmethod, the rationality theorem for klt pairs is proved by the Kawamata-Viehweg vanishing theorem, Hironaka's resolution theorem, and Shokurov's non-vanishing theorem (see, for example, [KM, §3.4]). Our proof only uses the vanishing theorem (Theorem 8.1). We need neither the non-vanishing theorem (cf. Theorem 12.1) nor Hironaka's resolution theorem in this section.

Theorem 15.1 (Rationality theorem). Let $X$ be a normal variety and let $B$ be an effective $\mathbb{Q}$-divisor on $X$ such that $K_{X}+B$ is $\mathbb{Q}$-Cartier. Let $\pi: X \rightarrow S$ be a projective morphism and let $H$ be a $\pi$-ample Cartier divisor on $X$. Assume that $K_{X}+B$ is not $\pi$-nef and that $r$ is a positive number such that

(1) $H+r\left(K_{X}+B\right)$ is $\pi$-nef but not $\pi$-ample, and

(2) $\left.\left(H+r\left(K_{X}+B\right)\right)\right|_{\mathrm{Nlc}(X, B)}$ is $\left.\pi\right|_{\mathrm{Nlc}(X, B)}$-ample.

Then $r$ is a rational number, and in reduced form, it has denominator at most $a(\operatorname{dim} X+1)$, where $a\left(K_{X}+B\right)$ is a Cartier divisor on $X$.

Before the proof of Theorem 15.1, we recall the following lemmas.

Lemma 15.2 (cf. [KM, Lemma 3.19]). Let $P(x, y)$ be a non-trivial polynomial of degree $\leq n$ and assume that $P$ vanishes for all sufficiently large integral solutions of $0<a y-r x<\varepsilon$ for some fixed positive integer a and positive $\varepsilon$ for some $r \in \mathbb{R}$. Then $r$ is rational, and in reduced form, it has denominator $\leq a(n+1) / \varepsilon$.

Proof. We assume that $r$ is irrational. Then an infinite number of integral points in the $(x, y)$-plane on each side of the line $a y-r x=0$ are closer than $\varepsilon /(n+2)$ to that line. So there is a large integral solution $\left(x^{\prime}, y^{\prime}\right)$ with $0<a y^{\prime}-r x^{\prime}<\varepsilon /(n+2)$. In this case, we see that

$$
\left(2 x^{\prime}, 2 y^{\prime}\right), \ldots,\left((n+1) x^{\prime},(n+1) y^{\prime}\right)
$$

are also solutions by hypothesis. So $y^{\prime} x-x^{\prime} y$ divides $P$, since $P$ and $y^{\prime} x-x^{\prime} y$ have $n+1$ common zeroes. We choose a smaller $\varepsilon$ and repeat the argument. We do this $n+1$ times to get a contradiction.

Now we assume that $r=u / v$ in lowest terms. For given $j$, let $\left(x^{\prime}, y^{\prime}\right)$ be a solution of $a y-r x=a j / v$. Note that an integral solution exists for every $j$. Then we have $a\left(y^{\prime}+k u\right)-r\left(x^{\prime}+a k v\right)=a j / v$ for all $k$. So, as above, if $a j / v<\varepsilon$, then $(a y-r x)-(a j / v)$ must divide $P$. So we can have at most $n$ such values of $j$. Thus $a(n+1) / v \geq \varepsilon$. 
Lemma 15.3. Let $C$ be a projective variety and let $D_{1}$ and $D_{2}$ be Cartier divisors on $X$. Consider the Hilbert polynomial

$$
P\left(u_{1}, u_{2}\right)=\chi\left(C, \mathcal{O}_{C}\left(u_{1} D_{1}+u_{2} D_{2}\right)\right) .
$$

If $D_{1}$ is ample, then $P\left(u_{1}, u_{2}\right)$ is a non-trivial polynomial of total degree $\leq \operatorname{dim} C$, because $P\left(u_{1}, 0\right)=h^{0}\left(C, \mathcal{O}_{C}\left(u_{1} D_{1}\right)\right) \not \equiv 0$ if $u_{1}$ is sufficiently large.

Proof of Theorem 15.1. Let $m$ be a positive integer such that $H^{\prime}=m H$ is $\pi$-very ample. If $H^{\prime}+r^{\prime}\left(K_{X}+B\right)$ is $\pi$-nef but not $\pi$-ample, and $\left.\left(H^{\prime}+r^{\prime}\left(K_{X}+B\right)\right)\right|_{\operatorname{Nlc}(X, B)}$ is $\left.\pi\right|_{\mathrm{Nlc}(X, B)}$-ample, then

$$
H+r\left(K_{X}+B\right)=\frac{1}{m}\left(H^{\prime}+r^{\prime}\left(K_{X}+B\right)\right) .
$$

This gives $r=(1 / m) r^{\prime}$. Thus, $r$ is rational if and only if $r^{\prime}$ is rational. Assume furthermore that $r^{\prime}$ has denominator $v$. Then $r$ has denominator dividing $m v$. Since $m$ can be an arbitrary sufficiently large integer, this implies that $r$ has denominator dividing $v$. Therefore, by replacing $H$ with $m H$, we can assume that $H$ is very ample over $S$.

For each $(p, q) \in \mathbb{Z}^{2}$, let $L(p, q)$ denote the relative base locus of the linear system $M(p, q)$ on $X$ (with the reduced scheme structure), that is,

$$
L(p, q)=\operatorname{Supp}\left(\operatorname{Coker}\left(\pi^{*} \pi_{*} \mathcal{O}_{X}(M(p, q)) \rightarrow \mathcal{O}_{X}(M(p, q))\right)\right),
$$

where $M(p, q)=p H+q a\left(K_{X}+B\right)$. By the definition, $L(p, q)=X$ if and only if $\pi_{*} \mathcal{O}_{X}(M(p, q))=0$.

Claim 1 (cf. [KM, Claim 3.20]). Let $\varepsilon$ be a positive number. For $(p, q)$ sufficiently large and $0<a q-r p<\varepsilon, L(p, q)$ is the same subset of $X$. We call it $L_{0}$. Let $I \subset \mathbb{Z}^{2}$ be the set of $(p, q)$ for which $0<a q-r p<1$ and $L(p, q)=L_{0}$. Then $I$ contains all sufficiently large $(p, q)$ with $0<a q-r p<1$.

Proof of Claim 1. We fix $\left(p_{0}, q_{0}\right) \in \mathbb{Z}^{2}$ such that $p_{0}>0$ and $0<a q_{0}-r p_{0}<1$. Since $H$ is $\pi$-very ample, there exists a positive integer $m_{0}$ such that $\mathcal{O}_{X}(m H+$ $\left.j a\left(K_{X}+B\right)\right)$ is $\pi$-generated for all $m>m_{0}$ and $0 \leq j \leq q_{0}-1$. Let $M$ be the round-up of

$$
\left(m_{0}+\frac{1}{r}\right) /\left(\frac{a}{r}-\frac{p_{0}}{q_{0}}\right)
$$

If $\left(p^{\prime}, q^{\prime}\right) \in \mathbb{Z}^{2}$ such that $0<a q^{\prime}-r p^{\prime}<1$ and $q^{\prime} \geq M+q_{0}-1$, then we can write

$$
p^{\prime} H+q^{\prime} a\left(K_{X}+B\right)=k\left(p_{0} H+q_{0} a\left(K_{X}+B\right)\right)+\left(l H+j a\left(K_{X}+B\right)\right)
$$


for some $k \geq 0,0 \leq j \leq q_{0}-1$ and $l>m_{0}$, because we can uniquely write $q^{\prime}=k q_{0}+j$ with $0 \leq j \leq q_{0}-1$. Thus, we have $k q_{0} \geq M$. So, we obtain

$$
l=p^{\prime}-k p_{0}>\frac{a}{r} q^{\prime}-\frac{1}{r}-\left(k q_{0}\right) \frac{p_{0}}{q_{0}} \geq\left(\frac{a}{r}-\frac{p_{0}}{q_{0}}\right) M-\frac{1}{r} \geq m_{0} .
$$

Therefore, $L\left(p^{\prime}, q^{\prime}\right) \subset L\left(p_{0}, q_{0}\right)$. By noetherian induction, we obtain the desired closed subset $L_{0} \subset X$ and $I \subset \mathbb{Z}^{2}$.

Claim 2. We have $L_{0} \cap \operatorname{Nlc}(X, B)=\emptyset$.

Proof of Claim 2. We take $(\alpha, \beta) \in \mathbb{Q}^{2}$ such that $\alpha>0, \beta>0$, and $\beta a / \alpha>r$ is sufficiently close to $r$. Then $\left.\left(\alpha H+\beta a\left(K_{X}+B\right)\right)\right|_{\mathrm{Nlc}(X, B)}$ is $\left.\pi\right|_{\operatorname{Nlc}(X, B) \text {-ample }}$ because $\left.\left(H+r\left(K_{X}+B\right)\right)\right|_{\mathrm{Nlc}(X, B)}$ is $\left.\pi\right|_{\mathrm{Nlc}(X, B)}$-ample. If $0<a q-r p<1$ and $(p, q) \in \mathbb{Z}^{2}$ is sufficiently large, then

$$
M(p, q)=m M(\alpha, \beta)+(M(p, q)-m M(\alpha, \beta))
$$

where $M(p, q)-m M(\alpha, \beta)$ is $\pi$-very ample, and

$$
\left.m\left(\alpha H+\beta a\left(K_{X}+B\right)\right)\right|_{\mathrm{Nlc}(X, B)}
$$

is also $\left.\pi\right|_{\mathrm{Nlc}(X, B)}$-very ample. This can be checked by the same argument as in the proof of Claim 1. Therefore, $\mathcal{O}_{\mathrm{Nlc}(X, B)}(M(p, q))$ is $\pi$-very ample. Since

$$
\pi_{*} \mathcal{O}_{X}(M(p, q)) \rightarrow \pi_{*} \mathcal{O}_{\mathrm{Nlc}(X, B)}(M(p, q))
$$

is surjective by the vanishing theorem (Theorem 8.1), we obtain $L(p, q) \cap \operatorname{Nlc}(X, B)$ $=\emptyset$. We note that

$$
M(p, q)-\left(K_{X}+B\right)=p H+(q a-1)\left(K_{X}+B\right)
$$

is $\pi$-ample because $(p, q)$ is sufficiently large and $a q-r p<1$. By Claim 1 , we have $L_{0} \cap \operatorname{Nlc}(X, B)=\emptyset$.

Claim 3. Assume that $r$ is either irrational, or rational and has denominator $>a(n+1)$ in reduced form, where $n=\operatorname{dim} X$. Then, for $(p, q)$ sufficiently large and $0<a q-r p<1, \mathcal{O}_{X}(M(p, q))$ is $\pi$-generated at the generic point of every lc center of $(X, B)$.

Proof of Claim 3. We note that

$$
M(p, q)-\left(K_{X}+B\right)=p H+(q a-1)\left(K_{X}+B\right) .
$$

If $a q-r p<1$ and $(p, q)$ is sufficiently large, then $M(p, q)-\left(K_{X}+B\right)$ is $\pi$-ample. Let $C$ be an lc center of $(X, B)$. We can assume $C \cap \operatorname{Nlc}(X, B)=\emptyset$ by Claim 2 . 
O. FuJino

Then $P_{C_{\eta}}(p, q)=\chi\left(C_{\eta}, \mathcal{O}_{C_{\eta}}(M(p, q))\right)$ is a non-zero polynomial of degree at most $\operatorname{dim} C_{\eta} \leq \operatorname{dim} X$ by Lemma 15.3. Note that $C_{\eta}$ is the generic fiber of $C \rightarrow \pi(C)$. By Lemma 15.2, there exists $(p, q)$ such that $P_{C_{\eta}}(p, q) \neq 0,(p, q)$ is sufficiently large, and $0<a q-r p<1$. By the $\pi$-ampleness of $M(p, q)-\left(K_{X}+B\right)$,

$$
P_{C_{\eta}}(p, q)=\chi\left(C_{\eta}, \mathcal{O}_{C_{\eta}}(M(p, q))\right)=h^{0}\left(C_{\eta}, \mathcal{O}_{C_{\eta}}(M(p, q))\right),
$$

and

$$
\pi_{*} \mathcal{O}_{X}(M(p, q)) \rightarrow \pi_{*} \mathcal{O}_{C}(M(p, q))
$$

is surjective by Theorem 8.1. We note that $C \cap \operatorname{Nlc}(X, B)=\emptyset$. Therefore, $\mathcal{O}_{X}(M(p, q))$ is $\pi$-generated at the generic point of $C$. By combining this with Claim $1, \mathcal{O}_{X}(M(p, q))$ is $\pi$-generated at the generic point of every lc center of $(X, B)$ if $(p, q)$ is sufficiently large with $0<a q-r p<1$. This yields Claim 3.

Note that $\mathcal{O}_{X}(M(p, q))$ is not $\pi$-generated for $(p, q) \in I$ because $M(p, q)$ is not $\pi$-nef. Therefore, $L_{0} \neq \emptyset$. We shrink $S$ to an affine open subset intersecting $\pi\left(L_{0}\right)$. Let $D_{1}, \ldots, D_{n+1}$ be general members of

$$
\pi_{*} \mathcal{O}_{X}\left(M\left(p_{0}, q_{0}\right)\right)=H^{0}\left(X, \mathcal{O}_{X}\left(M\left(p_{0}, q_{0}\right)\right)\right)
$$

with $\left(p_{0}, q_{0}\right) \in I$. We can check that $K_{X}+B+\sum_{i=1}^{n+1} D_{i}$ is not lc at the generic point of every irreducible component of $L_{0}$ by Lemma 13.2. On the other hand, $K_{X}+B+\sum_{i=1}^{n+1} D_{i}$ is lc outside $L_{0} \cup \operatorname{Nlc}(X, B)$ since $D_{i}$ is a general member of $\left|M\left(p_{0}, q_{0}\right)\right|$ for every $i$. Let $0<c<1$ be the log canonical threshold of $(X, B)$ with respect to $D=\sum_{i=1}^{n+1} D_{i}$ outside $\operatorname{Nlc}(X, B)$. Note that $c>0$ by Claim 3. Thus, the pair $(X, B+c D)$ has some lc centers contained in $L_{0}$. Let $C$ be an lc center contained in $L_{0}$. We note that $\mathcal{J}_{\mathrm{NLC}}(X, B+c D)=\mathcal{J}_{\mathrm{NLC}}(X, B)$ by Proposition 7.5 and $C \cap \operatorname{Nlc}(X, B+c D)=C \cap \operatorname{Nlc}(X, B)=\emptyset$. We consider

$$
K_{X}+B+c D=c(n+1) p_{0} H+\left(1+c(n+1) q_{0} a\right)\left(K_{X}+B\right) .
$$

Thus we have

$$
\begin{aligned}
p H+q a\left(K_{X}+B\right)-\left(K_{X}+B+c D\right) & \\
& =\left(p-c(n+1) p_{0}\right) H+\left(q a-\left(1+c(n+1) q_{0} a\right)\right)\left(K_{X}+B\right) .
\end{aligned}
$$

If $p$ and $q$ are large enough and $0<a q-r p \leq a q_{0}-r p_{0}$, then

$$
p H+q a\left(K_{X}+B\right)-\left(K_{X}+B+c D\right)
$$

is $\pi$-ample, because

$$
\begin{aligned}
& \left(p-c(n+1) p_{0}\right) H+\left(q a-\left(1+c(n+1) q_{0} a\right)\right)\left(K_{X}+B\right) \\
& =\left(p-(1+c(n+1)) p_{0}\right) H+\left(q a-(1+c(n+1)) q_{0} a\right)\left(K_{X}+B\right)+p_{0} H+\left(q_{0} a-1\right)\left(K_{X}+B\right) .
\end{aligned}
$$


Suppose that $r$ is not rational. There must be arbitrarily large $(p, q)$ such that $0<a q-r p<\varepsilon=a q_{0}-r p_{0}$ and $\chi\left(C_{\eta}, \mathcal{O}_{C_{\eta}}(M(p, q))\right) \neq 0$ by Lemma 15.2, because $P_{C_{\eta}}(p, q)=\chi\left(C_{\eta}, \mathcal{O}_{C_{\eta}}(M(p, q))\right)$ is a non-trivial polynomial of degree at most $\operatorname{dim} C_{\eta}$ by Lemma 15.3. Since $M(p, q)-\left(K_{X}+B+c D\right)$ is $\pi$-ample by $0<a q-r p<a q_{0}-r p_{0}$, we have $h^{0}\left(C_{\eta}, \mathcal{O}_{C_{\eta}}(M(p, q))\right)=\chi\left(C_{\eta}, \mathcal{O}_{C_{\eta}}(M(p, q))\right) \neq 0$ by the vanishing theorem (Theorem 8.1). By that theorem

$$
\pi_{*} \mathcal{O}_{X}(M(p, q)) \rightarrow \pi_{*} \mathcal{O}_{C}(M(p, q))
$$

is surjective because $M(p, q)-\left(K_{X}+B+c D\right)$ is $\pi$-ample. We note that $C \cap$ $\operatorname{Nlc}(X, B+c D)=\emptyset$. Thus $C$ is not contained in $L(p, q)$. Therefore, $L(p, q)$ is a proper subset of $L\left(p_{0}, q_{0}\right)=L_{0}$, giving the desired contradiction. So now we know that $r$ is rational.

We next suppose that the assertion of the theorem concerning the denominator of $r$ is false. We choose $\left(p_{0}, q_{0}\right) \in I$ such that $a q_{0}-r p_{0}$ is maximum, say $d / v$. If $0<a q-r p \leq d / v$ and $(p, q)$ is sufficiently large, then $\chi\left(C_{\eta}, \mathcal{O}_{C_{\eta}}(M(p, q))\right)=h^{0}\left(C_{\eta}, \mathcal{O}_{C_{\eta}}(M(p, q))\right)$ since $M(p, q)-\left(K_{X}+B+c D\right)$ is $\pi$-ample. There exists sufficiently large $(p, q)$ in the strip $0<a q-r p<1$ with $\varepsilon=1$ for which $h^{0}\left(C_{\eta}, \mathcal{O}_{C_{\eta}}(M(p, q))\right)=\chi\left(C_{\eta}, \mathcal{O}_{C_{\eta}}(M(p, q))\right) \neq 0$ by Lemma 15.2 since $\chi\left(C_{\eta}, \mathcal{O}_{C_{\eta}}(M(p, q))\right)$ is a non-trivial polynomial of degree at most $\operatorname{dim} C_{\eta}$ by Lemma 15.3. Note that $a q-r p \leq d / v=a q_{0}-r p_{0}$ automatically for $(p, q) \in I$. Since

$$
\pi_{*} \mathcal{O}_{X}(M(p, q)) \rightarrow \pi_{*} \mathcal{O}_{C}(M(p, q))
$$

is surjective by the $\pi$-ampleness of $M(p, q)-\left(K_{X}+B+c D\right)$, we obtain the desired contradiction for the same reason as above. This finishes the proof of the rationality theorem.

We close this section with an important remark, which is indispensable for the proof of the cone theorem (Theorem 16.6).

Remark 15.4. In Theorem 15.1, it is sufficient to assume that $B$ is an effective $\mathbb{R}$-divisor on $X$ such that $K_{X}+B$ is $\mathbb{R}$-linearly equivalent to a $\mathbb{Q}$-Cartier $\mathbb{Q}$-divisor $\omega$ on $X$ with $a \omega$ Cartier. All we have to do is to replace $a\left(K_{X}+B\right)$ with $a \omega$ in the proof of Theorem 15.1. We need this generalization in the proof of Theorem 16.6.

\section{$\S 16$. Cone theorem}

The main theorem of this section is the cone theorem. Before we state it, let us fix the notation. 
Definition 16.1. Let $X$ be a normal variety and let $B$ be an effective $\mathbb{R}$-divisor on $X$ such that $K_{X}+B$ is $\mathbb{R}$-Cartier. Let $\pi: X \rightarrow S$ be a projective morphism. We put

$$
\overline{N E}(X / S)_{\mathrm{Nlc}(X, B)}=\operatorname{Im}(\overline{N E}(\operatorname{Nlc}(X, B) / S) \rightarrow \overline{N E}(X / S)) .
$$

For an $\mathbb{R}$-Cartier $\mathbb{R}$-divisor $D$, we define

$$
D_{\geq 0}=\left\{z \in N_{1}(X / S) \mid D \cdot z \geq 0\right\} .
$$

Similarly, we can define $D_{>0}, D_{\leq 0}$, and $D_{<0}$. We also define

$$
D^{\perp}=\left\{z \in N_{1}(X / S) \mid D \cdot z=0\right\}
$$

We use the notation

$$
\overline{N E}(X / S)_{D \geq 0}=\overline{N E}(X / S) \cap D_{\geq 0},
$$

and similarly for $>0, \leq 0$, and $<0$.

Definition 16.2. An extremal face of $\overline{N E}(X / S)$ is a non-zero subcone $F \subset$ $\overline{N E}(X / S)$ such that $z, z^{\prime} \in F$ and $z+z^{\prime} \in F$ imply that $z, z^{\prime} \in F$. Equivalently, $F=\overline{N E}(X / S) \cap H^{\perp}$ for some $\pi$-nef $\mathbb{R}$-divisor $H$, which is called a supporting function of $F$. An extremal ray is a one-dimensional extremal face.

(1) An extremal face $F$ is called $\left(K_{X}+B\right)$-negative if

$$
F \cap \overline{N E}(X / S)_{K_{X}+B \geq 0}=\{0\} .
$$

(2) An extremal face $F$ is called rational if we can choose a $\pi$-nef $\mathbb{Q}$-divisor $H$ as a support function of $F$.

(3) An extremal face $F$ is called relatively ample at $\operatorname{Nlc}(X, B)$ if

$$
F \cap \overline{N E}(X / S)_{\mathrm{Nlc}(X, B)}=\{0\} .
$$

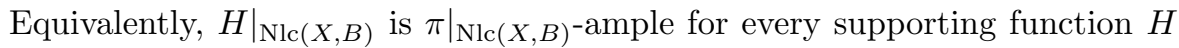
of $F$.

(4) An extremal face $F$ is called contractible at $\operatorname{Nlc}(X, B)$ if it has a rational

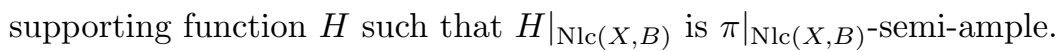

Remark 16.3. If $X$ is complete but non-projective, then it sometimes happens that $\overline{N E}(X)=N_{1}(X)$ even when $X$ is smooth (cf. [FP]). Therefore, the projectivity is crucial for the log minimal model program.

The following theorem is a direct consequence of Theorem 13.1. 
Theorem 16.4 (Contraction theorem). Let $X$ be a normal variety, let $B$ be an effective $\mathbb{R}$-divisor on $X$ such that $K_{X}+B$ is $\mathbb{R}$-Cartier, and let $\pi: X \rightarrow S$ be a projective morphism. Let $H$ be a $\pi$-nef Cartier divisor such that $F=H^{\perp} \cap$ $\overline{N E}(X / S)$ is $\left(K_{X}+B\right)$-negative and contractible at $\operatorname{Nlc}(X, B)$. Then there exists a projective morphism $\varphi_{F}: X \rightarrow Y$ over $S$ with the following properties:

(1) Let $C$ be an integral curve on $X$ such that $\pi(C)$ is a point. Then $\varphi_{F}(C)$ is a point if and only if $[C] \in F$.

(2) $\mathcal{O}_{Y} \simeq\left(\varphi_{F}\right)_{*} \mathcal{O}_{X}$.

(3) Let $L$ be a line bundle on $X$ such that $L \cdot C=0$ for every curve $C$ with $[C] \in F$.

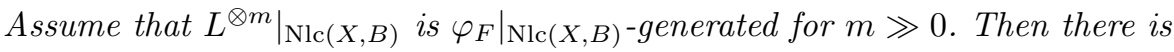
a line bundle $L_{Y}$ on $Y$ such that $L \simeq \varphi_{F}^{*} L_{Y}$.

Proof. By the assumption, $q H-\left(K_{X}+B\right)$ is $\pi$-ample for some positive integer

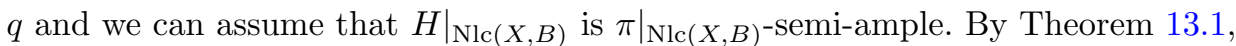
$\mathcal{O}_{X}(m H)$ is $\pi$-generated for some $m>0$. We take the Stein factorization of the associated morphism. Then we have the contraction morphism $\varphi_{F}: X \rightarrow Y$ with the properties (1) and (2).

We consider $\varphi_{F}: X \rightarrow Y$ and $\overline{N E}(X / Y)$. Then $\overline{N E}(X / Y)=F, L$ is numerically trivial over $Y$, and $-\left(K_{X}+B\right)$ is $\varphi_{F}$-ample. Applying the base point free theorem (cf. Theorem 13.1) over $Y$, both $L^{\otimes m}$ and $L^{\otimes(m+1)}$ are pull-backs of line bundles on $Y$. Their difference gives a line bundle $L_{Y}$ such that $L \simeq \varphi_{F}^{*} L_{Y}$.

Example 16.5. Let $S$ be a cone over a smooth cubic curve and let $\pi: X \rightarrow S$ be the blow-up at the vertex of $S$. Then $K_{X}+E=\pi^{*} K_{S}$, where $E$ is the $\pi$ exceptional curve. We put $B=2 E$ and consider the pair $(X, B)$. In this case, $\varphi_{F}=\pi: X \rightarrow Y=S$ with $F=0^{\perp} \cap \overline{N E}(X / S)=\overline{N E}(X / S)=\mathbb{R}_{\geq 0}[E]$ is an example of contraction morphisms in Theorem 16.4.

The time is ripe to state one of the main theorems in this paper.

Theorem 16.6 (Cone theorem). Let $X$ be a normal variety, let $B$ be an effective $\mathbb{R}$-divisor on $X$ such that $K_{X}+B$ is $\mathbb{R}$-Cartier, and let $\pi: X \rightarrow S$ be a projective morphism. Then we have the following properties:

(1) $\overline{N E}(X / S)=\overline{N E}(X / S)_{K_{X}+B \geq 0}+\overline{N E}(X / S)_{\mathrm{Nlc}(X, B)}+\sum R_{j}$, where $R_{j}$ 's are the $\left(K_{X}+B\right)$-negative extremal rays of $\overline{N E}(X / S)$ that are rational and relatively ample at $\operatorname{Nlc}(X, B)$. In particular, each $R_{j}$ is spanned by an integral curve $C_{j}$ on $X$ such that $\pi\left(C_{j}\right)$ is a point.

(2) Let $H$ be a $\pi$-ample $\mathbb{R}$-divisor on $X$. Then there are only finitely many $R_{j}$ 's included in $\left(K_{X}+B+H\right)_{<0}$. In particular, the $R_{j}$ 's are discrete in the halfspace $\left(K_{X}+B\right)_{<0}$. 
(3) Let $F$ be a $\left(K_{X}+B\right)$-negative extremal face of $\overline{N E}(X / S)$ that is relatively ample at $\operatorname{Nlc}(X, B)$. Then $F$ is a rational face. In particular, $F$ is contractible at $\operatorname{Nlc}(X, B)$.

Proof. First, we assume that $K_{X}+B$ is $\mathbb{R}$-linearly equivalent to a $\mathbb{Q}$-Cartier $\mathbb{Q}$ divisor on $X$ (see Remark 15.4). We can assume that $\operatorname{dim}_{\mathbb{R}} N_{1}(X / S) \geq 2$ and $K_{X}+B$ is not $\pi$-nef. Otherwise, the theorem is obvious.

Step 1. We have

$$
\overline{N E}(X / S)=\overline{\overline{N E}(X / S)_{K_{X}+B \geq 0}+\overline{N E}(X / S)_{\mathrm{Nlc}(X, B)}+\sum_{F} F},
$$

where $F$ 's vary among all rational proper $\left(K_{X}+B\right)$-negative faces that are relatively ample at $\operatorname{Nlc}(X, B)$ and the overline denotes closure in the real topology.

Indeed, put

$$
\mathfrak{B}=\overline{\overline{N E}(X / S)_{K_{X}+B \geq 0}+\overline{N E}(X / S)_{\mathrm{Nlc}(X, B)}+\sum_{F} F} .
$$

It is clear that $\overline{N E}(X / S) \supset \mathfrak{B}$. We note that each $F$ is spanned by curves on $X$ mapped to points on $S$ by Theorem 16.4(1). Supposing $\overline{N E}(X / S) \neq \mathfrak{B}$, we shall derive a contradiction. There is a separating function $M$ which is Cartier and is not a multiple of $K_{X}+B$ in $N^{1}(X / S)$ such that $M>0$ on $\mathfrak{B} \backslash\{0\}$ and $M \cdot z_{0}<0$ for some $z_{0} \in \overline{N E}(X / S)$. Let $C$ be the dual cone of $\overline{N E}(X / S)_{K_{X}+B \geq 0}$, that is,

$$
C=\left\{D \in N^{1}(X / S) \mid D \cdot z \geq 0 \text { for } z \in \overline{N E}(X / S)_{K_{X}+B \geq 0}\right\}
$$

Then we see that $C$ is generated by $\pi$-nef divisors and $K_{X}+B$. Since $M>0$ on $\overline{N E}(X / S)_{K_{X}+B \geq 0} \backslash\{0\}, M$ is in the interior of $C$, and hence there exists a $\pi$-ample $\mathbb{Q}$-Cartier $\mathbb{Q}$-divisor $A$ such that $M-A=L^{\prime}+p\left(K_{X}+B\right)$ in $N^{1}(X / S)$, where $L^{\prime}$ is a $\pi$-nef $\mathbb{Q}$-Cartier $\mathbb{Q}$-divisor on $X$ and $p$ is a non-negative rational number. Therefore, $M$ is expressed in the form $M=H+p\left(K_{X}+B\right)$ in $N^{1}(X / S)$, where $H=A+L^{\prime}$ is a $\pi$-ample $\mathbb{Q}$-Cartier $\mathbb{Q}$-divisor. The rationality theorem (see Theorem 15.1) implies that there exists a positive rational number $r<p$ such that $L=H+r\left(K_{X}+B\right)$ is $\pi$-nef but not $\pi$-ample, and $\left.L\right|_{\mathrm{Nlc}(X, B)}$ is $\left.\pi\right|_{\mathrm{Nlc}(X, B)}$-ample. Note that $L \neq 0$ in $N^{1}(X / S)$, since $M$ is not a multiple of $K_{X}+B$. Thus the extremal face $F_{L}$ associated to the supporting function $L$ is contained in $\mathfrak{B}$, which implies $M>0$ on $F_{L}$. Therefore, $p<r$, a contradiction.

Step 2. In the equality of Step 1, we can assume that every extremal face $F$ is one-dimensional. 
Indeed, let $F$ be a rational proper $\left(K_{X}+B\right)$-negative extremal face that is relatively ample at $\operatorname{Nlc}(X, B)$, and assume that $\operatorname{dim} F \geq 2$. Let $\varphi_{F}: X \rightarrow W$ be the associated contraction. Note that $-\left(K_{X}+B\right)$ is $\varphi_{F}$-ample. By Step 1 ,

$$
F=\overline{N E}(X / W)=\overline{\sum_{G} G},
$$

where the $G$ 's are the rational proper $\left(K_{X}+B\right)$-negative extremal faces of the cone $\overline{N E}(X / W)$. We note that $\overline{N E}(X / W)_{\mathrm{Nlc}(X, B)}=0$ because $\varphi_{F}$ embeds $\operatorname{Nlc}(X, B)$ into $W$. The $G$ 's are also $\left(K_{X}+B\right)$-negative extremal faces of $\overline{N E}(X / S)$ that are ample at $\operatorname{Nlc}(X, B)$, and $\operatorname{dim} G<\operatorname{dim} F$. By induction, we obtain

$$
\overline{N E}(X / S)=\overline{\overline{N E}(X / S)_{K_{X}+B \geq 0}+\overline{N E}(X / S)_{\mathrm{Nlc}(X, B)}+\sum R_{j}},
$$

where the $R_{j}$ 's are $\left(K_{X}+B\right)$-negative rational extremal rays. Note that no $R_{j}$ intersects $\overline{N E}(X / S)_{\mathrm{Nlc}}(X, B)$.

Step 3. The contraction theorem (cf. Theorem 16.4) guarantees that for each extremal ray $R_{j}$ there exists a reduced irreducible curve $C_{j}$ on $X$ such that $\left[C_{j}\right] \in R_{j}$. Let $\psi_{j}: X \rightarrow W_{j}$ be the contraction morphism of $R_{j}$, and let $A$ be a $\pi$-ample Cartier divisor. We set

$$
r_{j}=-\frac{A \cdot C_{j}}{\left(K_{X}+B\right) \cdot C_{j}} .
$$

Then $A+r_{j}\left(K_{X}+B\right)$ is $\psi_{j}$-nef but not $\psi_{j}$-ample, and $\left.\left(A+r_{j}\left(K_{X}+B\right)\right)\right|_{\operatorname{Nlc}(X, B)}$ is $\left.\psi_{j}\right|_{\mathrm{Nlc}(X, B)}$-ample. By the rationality theorem (see Theorem 15.1), expressing $r_{j}=$ $u_{j} / v_{j}$ with $u_{j}, v_{j} \in \mathbb{Z}_{>0}$ and $\left(u_{j}, v_{j}\right)=1$, we have the inequality $v_{j} \leq a(\operatorname{dim} X+1)$.

Step 4. Now take $\pi$-ample Cartier divisors $H_{1}, \ldots, H_{\rho-1}$ such that $K_{X}+B$ and the $H_{i}$ 's form a basis of $N^{1}(X / S)$, where $\rho=\operatorname{dim}_{\mathbb{R}} N^{1}(X / S)$. By Step 3, the intersections of the extremal rays $R_{j}$ with the hyperplane

$$
\left\{z \in N_{1}(X / S) \mid a\left(K_{X}+B\right) \cdot z=-1\right\}
$$

in $N_{1}(X / S)$ lie on the lattice

$$
\Lambda=\left\{z \in N_{1}(X / S) \mid a\left(K_{X}+B\right) \cdot z=-1, H_{i} \cdot z \in(a(a(\operatorname{dim} X+1)) !)^{-1} \mathbb{Z}\right\} .
$$

This implies that the extremal rays are discrete in the half-space

$$
\left\{z \in N_{1}(X / S) \mid\left(K_{X}+B\right) \cdot z<0\right\}
$$

Thus we can omit the closure sign from the formula (\$) and this completes the proof of (1) when $K_{X}+B$ is $\mathbb{R}$-linearly equivalent to a $\mathbb{Q}$-Cartier $\mathbb{Q}$-divisor. 
Step 5. Let $H$ be a $\pi$-ample $\mathbb{R}$-divisor on $X$. We choose $0<\varepsilon_{i} \ll 1$ for $1 \leq$ $i \leq \rho-1$ such that $H-\sum_{i=1}^{\rho-1} \varepsilon_{i} H_{i}$ is $\pi$-ample. Then the $R_{j}$ 's included in $\left(K_{X}+B+H\right)_{<0}$ correspond to some elements of the above lattice $\Lambda$ for which $\sum_{i=1}^{\rho-1} \varepsilon_{i} H_{i} \cdot z<1 / a$. Therefore, we obtain (2).

Step 6. Let $F$ be a $\left(K_{X}+B\right)$-negative extremal face as in (3). The vector space $V=F^{\perp} \subset N^{1}(X / S)$ is defined over $\mathbb{Q}$ because $F$ is generated by some of the $R_{j}$ 's. There exists a $\pi$-ample $\mathbb{R}$-divisor $H$ such that $F$ is contained in $\left(K_{X}+B+H\right)_{<0}$. Let $\langle F\rangle$ be the vector space spanned by $F$. We put

$$
W_{F}=\overline{N E}(X / S)_{K_{X}+B+H \geq 0}+\overline{N E}(X / S)_{\mathrm{Nlc}(X, B)}+\sum_{R_{j} \not \subset F} R_{j} .
$$

Then $W_{F}$ is a closed cone, $\overline{N E}(X / S)=W_{F}+F$, and $W_{F} \cap\langle F\rangle=\{0\}$. The supporting functions of $F$ are the elements of $V$ that are positive on $W_{F} \backslash\{0\}$. This is a non-empty open set and thus it contains a rational element that, after scaling, gives a $\pi$-nef Cartier divisor $L$ such that $F=L^{\perp} \cap \overline{N E}(X / S)$. Therefore, $F$ is rational. So, we have (3).

From now on, $K_{X}+B$ is $\mathbb{R}$-Cartier.

Step 7. Let $H$ be a $\pi$-ample $\mathbb{R}$-divisor on $X$. We shall prove (2). We assume, for a contradiction, that there are infinitely many $R_{j}$ 's in $\left(K_{X}+B+H\right)_{<0}$. There exists an affine open subset $U$ of $S$ such that $\overline{N E}\left(\pi^{-1}(U) / U\right)$ has infinitely many $\left(K_{X}+B+H\right)$-negative extremal rays. So, we shrink $S$, and can assume that $S$ is affine. We can write $H=E+H^{\prime}$ where $H^{\prime}$ is $\pi$-ample, $\mathcal{J}_{\mathrm{NLC}}(X, B+E)=$ $\mathcal{J}_{\mathrm{NLC}}(X, B)$, and $K_{X}+B+E$ is $\mathbb{R}$-linearly equivalent to a $\mathbb{Q}$-Cartier $\mathbb{Q}$-divisor. Since $K_{X}+B+H=K_{X}+B+E+H^{\prime}$, we have

$$
\overline{N E}(X / S)=\overline{N E}(X / S)_{K_{X}+B+H \geq 0}+\overline{N E}(X / S)_{\mathrm{Nlc}(X, B)}+\sum_{\text {finite }} R_{j} .
$$

This is a contradiction. Thus, we obtain (2). The statement (1) is a direct consequence of (2). Of course, (3) holds by Step 6 once we obtain (1).

So, the proof of the cone theorem is complete.

We close this section with the following elementary example.

Example 16.7. We consider $Y=\mathbb{P}^{1} \times \mathbb{P}^{1}$. Let $\pi_{i}: Y \rightarrow \mathbb{P}^{1}$ be the $i$-th projection for $i=1,2$. Let $F_{i}$ be a fiber of $\pi_{i}$ for $i=1,2$. We put $P=F_{1} \cap F_{2}$ and consider the blow-up $f: X \rightarrow Y$ at $P$. Let $E$ be the exceptional curve of $f$ and $C_{i}=f_{*}^{-1} F_{i}$ for $i=1,2$. In this situation, we can check that $-K_{X}$ is ample, $\rho(X)=3$, and

$$
\overline{N E}(X)=\mathbb{R}_{\geq 0}\left[C_{1}\right]+\mathbb{R}_{\geq 0}\left[C_{2}\right]+\mathbb{R}_{\geq 0}[E] .
$$


We put

Then we have

$$
B=\frac{3}{2} E+\frac{1}{2} C_{1}+C_{2}
$$

$$
\overline{N E}(X)=\overline{N E}(X)_{K_{X}+B \geq 0}+\overline{N E}(X)_{\mathrm{Nlc}(X, B)}+\mathbb{R}_{\geq 0}\left[C_{2}\right],
$$

where

$$
\overline{N E}(X)_{\mathrm{Nlc}(X, B)}=\mathbb{R}_{\geq 0}[E], \quad \overline{N E}(X)_{K_{X}+B \geq 0}=\mathbb{R}_{\geq 0}\left[C_{1}\right]
$$

and

$$
C_{2} \cdot\left(K_{X}+B\right)<0
$$

\section{$\S 17$. Base point free theorem revisited}

This section is a supplement to the base point free theorem (Theorem 13.1). In the recent log minimal model program (cf. [S3], [BCHM], and so on), we frequently use $\mathbb{R}$-divisors. Therefore, the following theorem is useful.

Theorem 17.1 (Base point free theorem for $\mathbb{R}$-divisors). Let $X$ be a normal variety and let $B$ be an effective $\mathbb{R}$-divisor on $X$ such that $(X, B)$ is log canonical, and let $\pi: X \rightarrow S$ be a projective morphism onto a variety $S$. Let $D$ be a $\pi$-nef $\mathbb{R}$-Cartier $\mathbb{R}$-divisor on $X$ such that aD- $\left(K_{X}+B\right)$ is $\pi$-ample for some real number $a>0$. Then $D$ is $\pi$-semi-ample.

Proof. We can assume that $a=1$ by replacing $D$ with $a D$. We put

$$
F=\{z \in \overline{N E}(X / S) \mid D \cdot z=0\} .
$$

Then $F$ is a face of $\overline{N E}(X / S)$ and $\left(K_{X}+B\right) \cdot z<0$ for $z \in F$. We claim that $F$ contains only finitely many $\left(K_{X}+B\right)$-negative extremal rays $R_{1}, \ldots, R_{k}$ of $\overline{N E}(X / S)$. If $F$ contains infinitely many $\left(K_{X}+B\right)$-negative extremal rays of $\overline{N E}(X / S)$, then this also holds after shrinking $S$ suitably. Therefore, we can assume that $S$ is affine. In this situation, $X$ is quasi-projective. We take a general small $\pi$-ample $\mathbb{Q}$-divisor $A$ on $X$ such that $D-\left(K_{X}+B+A\right)$ is $\pi$-ample and $(X, B+A)$ is log canonical. Let $R$ be a $\left(K_{X}+B\right)$-negative extremal ray such that $R \subset F$. Then $R$ is a $\left(K_{X}+B+A\right)$ negative extremal ray since $D \cdot R=0$ and $D-\left(K_{X}+B+A\right)$ is $\pi$-ample. On the other hand, there are only finitely many $\left(K_{X}+B+A\right)$-negative extremal rays in $\overline{N E}(X / S)$ by Theorem 16.6(2). This is a contradiction. Therefore, $F$ is spanned by the extremal rays $R_{1}, \ldots, R_{k}$. We consider the finite-dimensional real vector space $V=\bigoplus_{j} \mathbb{R} D_{j}$, where $\sum_{j} D_{j}=\operatorname{Supp} D$ is the irreducible decomposition. Then

$$
\mathcal{R}=\{E \in V \mid E \text { is } \mathbb{R} \text {-Cartier and } E \cdot z=0 \text { for every } z \in F\}
$$


is a rational affine subspace of $V$ and $D \in \mathcal{R}$. Thus, we can find positive real numbers $r_{1}, \ldots, r_{m}$ and $\pi$-nef $\mathbb{Q}$-Cartier $\mathbb{Q}$-divisors $E_{1}, \ldots, E_{m}$ such that $D=$ $\sum_{i=1}^{m} r_{i} E_{i}$ and $E_{i}-\left(K_{X}+B\right)$ is $\pi$-ample for every $i$ (cf. Step 6 in the proof of Theorem 16.6). By Theorem 13.1, $E_{i}$ is a $\pi$-semi-ample $\mathbb{Q}$-Cartier $\mathbb{Q}$-divisor for every $i$. Therefore, $D$ is $\pi$-semi-ample.

\section{$\S 18$. Lengths of extremal rays}

In this section, we discuss estimates of lengths of extremal rays. This is indispensable for the log minimal model program with scaling (see, for example, [BCHM]). Some results in this section have already been obtained in [Ko2], [Ko3], [Ka2], [S3], [S4], and [B1] with some extra assumptions. We note that the formulation of the main theorem of this section (cf. Theorem 18.10) is new.

Let us recall the following easy lemma.

Lemma 18.1 (cf. [S4, Lemma 1]). Let $(X, B)$ be a log canonical pair, where $B$ is an $\mathbb{R}$-divisor. Then there are positive real numbers $r_{i}$ and effective $\mathbb{Q}$-divisors $B_{i}$ for $1 \leq i \leq l$ and a positive integer $m$ such that $\sum_{i=1}^{l} r_{i}=1, K_{X}+B=$ $\sum_{i=1}^{l} r_{i}\left(K_{X}+B_{i}\right),\left(X, B_{i}\right)$ is lc for every $i$, and $m\left(K_{X}+B_{i}\right)$ is Cartier for every $i$.

Proof. Let $\sum_{k} D_{k}$ be the irreducible decomposition of Supp $B$. We consider the finite-dimensional real vector space $V=\bigoplus_{k} \mathbb{R} D_{k}$. We put

$$
\mathcal{Q}=\left\{D \in V \mid K_{X}+D \text { is } \mathbb{R} \text {-Cartier }\right\} .
$$

Then it is easy to see that $\mathcal{Q}$ is an affine subspace of $V$ defined over $\mathbb{Q}$. We put

$$
\mathcal{P}=\left\{D \in \mathcal{Q} \mid K_{X}+D \text { is log canonical }\right\} .
$$

Thus by the definition of $\log$ canonicity, it is also easy to check that $\mathcal{P}$ is a closed convex rational polytope in $V$. We note that $\mathcal{P}$ is compact in the classical topology of $V$. By the assumption, $B \in \mathcal{P}$. Therefore, we can find the desired $\mathbb{Q}$-divisors $B_{i} \in \mathcal{P}$ and positive real numbers $r_{i}$.

The next result is essentially due to [Ka2] and [S4, Proposition 1]. We will prove a more general result in Theorem 18.10 whose proof depends on Theorem 18.2 .

Theorem 18.2. Let $(X, B)$ be an lc pair and let $\pi: X \rightarrow S$ be a projective morphism onto a variety $S$. Let $R$ be a $\left(K_{X}+B\right)$-negative extremal ray of $\overline{N E}(X / S)$. Then we can find a rational curve $C$ on $X$ such that $[C] \in R$ and

$$
0<-\left(K_{X}+B\right) \cdot C \leq 2 \operatorname{dim} X .
$$


Proof. By shrinking $S$, we can assume that $S$ is quasi-projective. By replacing $\pi: X \rightarrow S$ with the extremal contraction $\varphi_{R}: X \rightarrow Y$ over $S$, we can assume that the relative Picard number $\rho(X / S)$ is 1 . In particular, $-\left(K_{X}+B\right)$ is $\pi$-ample. Let $K_{X}+B=\sum_{i=1}^{l} r_{i}\left(K_{X}+B_{i}\right)$ be as in Lemma 18.1. Without loss of generality, we can assume that $-\left(K_{X}+B_{1}\right)$ is $\pi$-ample and $-\left(K_{X}+B_{i}\right)=-s_{i}\left(K_{X}+B_{1}\right)$ in $N^{1}(X / S)$ with $s_{i} \leq 1$ for every $i \geq 2$. Thus, it is sufficient to find a rational curve $C$ such that $\pi(C)$ is a point and $-\left(K_{X}+B_{1}\right) \cdot C \leq 2 \operatorname{dim} X$. So, we can assume that $K_{X}+B$ is $\mathbb{Q}$-Cartier and lc. By Theorem 10.4, there is a birational morphism $f:\left(V, B_{V}\right) \rightarrow(X, B)$ such that $K_{V}+B_{V}=f^{*}\left(K_{X}+B\right), V$ is $\mathbb{Q}$-factorial, and $\left(V, B_{V}\right)$ is dlt. By [Ka2, Theorem 1] and [Ma, Theorem 10-2-1], we can find a rational curve $C^{\prime}$ on $V$ such that $-\left(K_{V}+B_{V}\right) \cdot C^{\prime} \leq 2 \operatorname{dim} V=2 \operatorname{dim} X$ and $C^{\prime}$ spans a $\left(K_{V}+B_{V}\right)$-negative extremal ray. By the projection formula, the $f$-image of $C^{\prime}$ is the desired rational curve.

Remark 18.3. It is conjectured that the estimate $\leq 2 \operatorname{dim} X$ in Theorem 18.2 can be replaced by $\leq \operatorname{dim} X+1$. When $X$ is smooth projective, this is true by Mori's famous result (cf. [Mo]). See, for example, [KM, Theorem 1.13]. When $X$ is a toric variety, it is also true by [F2] and [F5].

Remark 18.4. In the proof of Theorem 18.2, we need Kawamata's estimate on the length of an extremal rational curve (cf. [Ka2, Theorem 1] and [Ma, Theorem 10-2-1]). It depends on Mori's bend and break technique to create rational curves. So, we need the $\bmod p$ reduction technique there.

Remark 18.5. We give a remark on $[\mathrm{BCHM}]$. We use the same notation as in [BCHM, 3.8]. In the proof of [BCHM, Corollary 3.8.2], we can assume that $K_{X}+\Delta$ is klt by [BCHM, Lemma 3.7.4]. By perturbing the coefficients of $B$ slightly, we can further assume that $B$ is a $\mathbb{Q}$-divisor. By applying the usual cone theorem to the klt pair $(X, B)$, we deduce that there are only finitely many $\left(K_{X}+\Delta\right)$-negative extremal rays of $\overline{N E}(X / U)$. We note that [BCHM, Theorem 3.8.1] is only used in the proof of [BCHM, Corollary 3.8.2]. Therefore, we do not need the estimate of lengths of extremal rays in $[\mathrm{BCHM}]$. In particular, we do not need $\bmod p$ reduction arguments for the proof of the main results in [BCHM].

By the proof of Theorem 18.2, we have the following corollary.

Corollary 18.6. Let $(X, B)$ be a log canonical pair and let

$$
K_{X}+B=\sum_{i=1}^{l} r_{i}\left(K_{X}+B_{i}\right)
$$


and $m$ be as in Lemma 18.1. Let $\varphi: X \rightarrow Y$ be a projective surjective morphism with connected fibers such that the relative Picard number $\rho(X / Y)$ is 1 . Then we can find a curve $C$ on $X$ such that $C$ spans $N_{1}(X / Y)$ and

$$
-\left(K_{X}+B_{i}\right) \cdot C=\frac{n_{i}}{m}
$$

with $n_{i} \leq 2 m \operatorname{dim} X$ for every $i$. Of course,

$$
-\left(K_{X}+B\right) \cdot C=\sum_{i} \frac{r_{i} n_{i}}{m} \leq 2 \operatorname{dim} X .
$$

If $-\left(K_{X}+B_{i}\right)$ is $\varphi$-ample for some $i$, then we can find a rational curve $C$ in the above statement. Note that $\varphi$ is not necessarily assumed to be $a\left(K_{X}+B\right)$-negative extremal contraction.

The following important lemma is a very special case of [S3, 6.2. First Main Theorem].

Lemma 18.7. Let $(X, B)$ be a $\log$ canonical pair and let $\pi: X \rightarrow S$ be a projective morphism onto a variety $S$. Take $\sum_{k} D_{k}$ such that $\operatorname{Supp} B \subset \sum_{k} D_{k}$, where $D_{i}$ is an irreducible Weil divisor for every $i$ and $D_{i} \neq D_{j}$ for every $i \neq j$. Put

$$
\mathcal{P}=\left\{\sum_{k} d_{k} D_{k} \mid 0 \leq d_{k} \leq 1 \text { for all } k \text { and } K_{X}+\sum_{k} d_{k} D_{k} \text { is } l c\right\} .
$$

Then $\mathcal{P}$ is a closed convex rational polytope.

Let $\left\{R_{j}\right\}$ be any set of $\left(K_{X}+B\right)$-negative extremal rays of the lc pair $(X, B)$ over S. Put

$$
\mathcal{N}=\bigcap_{j}\left\{\sum_{k} d_{k} D_{k} \in \mathcal{P} \mid\left(K_{X}+\sum_{k} d_{k} D_{k}\right) \cdot R_{j} \geq 0\right\} .
$$

Then $\mathcal{N}$ is a closed convex subset of $\mathcal{P}$.

Take $B^{\prime} \in \mathcal{P}$. Let $\mathcal{F}$ be the minimal face of $\mathcal{P}$ containing $B^{\prime}$. Assume that $\left(K_{X}+B^{\prime}\right) \cdot R_{j}>0$ for every $j$. Then there is an open subset $U$ of $\mathcal{F}$ in the classical topology such that $B^{\prime} \in U \subset \mathcal{N} \cap \mathcal{F}$. In particular, we can write

$$
K_{X}+B^{\prime}=\sum_{i=1}^{d+1} r_{i}^{\prime}\left(K_{X}+B_{i}^{\prime}\right)
$$

with the following properties:
(a) $d=\operatorname{dim} \mathcal{F}$.
(b) $B_{i}^{\prime} \in \mathcal{F}$ for every $i$.
(c) $m^{\prime}\left(K_{X}+B_{i}^{\prime}\right)$ is Cartier for some positive integer $m^{\prime}$ for every $i$. 
(d) $\sum_{i=1}^{d+1} r_{i}^{\prime}=1$ and $0 \leq r_{i}^{\prime} \leq 1$ for every $i$.

(e) $\left(K_{X}+B_{i}^{\prime}\right) \cdot R_{j}>0$ for every $i$ and $j$.

Proof. It is obvious that $\mathcal{P}$ is a closed convex rational polytope (see the proof of Lemma 18.1). By definition, $\mathcal{N}$ is a closed convex subset of $\mathcal{P}$. Since $\mathcal{F}$ is a face of $\mathcal{P}$ and contains $B^{\prime}$, we can take a $d$-dimensional rational simplex spanned by $\Delta_{i}$ for $1 \leq i \leq d+1$ in $\mathcal{F}$ containing $B^{\prime}$ inside it. Thus, we can write

$$
K_{X}+B^{\prime}=\sum_{i=1}^{d+1} r_{i}\left(K_{X}+\Delta_{i}\right)
$$

with $\sum_{i=1}^{d+1} r_{i}=1$ and $0<r_{i}<1$ for every $i$, and $m\left(K_{X}+\Delta_{i}\right)$ with Cartier for every $i$, where $m$ is a positive integer.

We take an extremal ray $R_{j}$. By Corollary 18.6, we can find a curve $C_{j}$ on $X$ such that $C_{j}$ spans $R_{j}$ and that $m\left(K_{X}+\Delta_{i}\right) \cdot C_{j}=n_{i j}$ with $n_{i j} \geq-2 m \operatorname{dim} X$ for every $i$. By assumption, we have

$$
\left(K_{X}+B^{\prime}\right) \cdot C_{j}=\sum_{i} \frac{r_{i} n_{i j}}{m}>0
$$

We define

$$
\alpha=\inf \left\{\sum_{i} \frac{r_{i} n_{i}}{m}>0 \mid n_{i} \geq-2 m \operatorname{dim} X \text { and } n_{i} \in \mathbb{Z} \text { for every } i\right\} .
$$

Then we obtain $\alpha>0$. We put

$$
c=\frac{\alpha}{2 \operatorname{dim} X+\alpha+1}>0 .
$$

It is obvious that

$$
B^{\prime}+c\left(\Delta_{i}-B^{\prime}\right) \in \mathcal{F}
$$

for every $i$ since $0<c<1$ and that

$$
\left(K_{X}+B^{\prime}+c\left(\Delta_{i}-B^{\prime}\right)\right) \cdot C_{j}>0
$$

for every $i$ and $j$ by the definition of $c$. Thus, the $d$-dimensional simplex spanned by $B^{\prime}+c\left(\Delta_{i}-B^{\prime}\right)$ for $1 \leq i \leq d+1$ is contained in $\mathcal{N} \cap \mathcal{F}$ and contains $B^{\prime}$ in its interior. So, the interior of the above simplex is the desired open set contained in $\mathcal{N} \cap \mathcal{F}$. Thus, we can write

$$
K_{X}+B^{\prime}=\sum_{i=1}^{d+1} r_{i}^{\prime}\left(K_{X}+B_{i}^{\prime}\right)
$$

with the required properties. 
Remark 18.8. In [S3, 6.2. First Main Theorem], it is proved that $\mathcal{N}$ is a closed convex rational polytope. We refer the reader to [B2, Section 3] for the details. The arguments in [B2, Section 3] work for lc pairs by Theorem 18.2 (see, for example, [F11]).

By Corollary 18.6 and Lemma 18.7, Lemma 2.6 in [B1] holds for lc pairs. It may be useful for the log minimal model program with scaling. We follow Birkar's proof in $[\mathrm{BP}]$.

Theorem 18.9 (cf. [B1, Lemma 2.6]). Let $(X, B)$ be an lc pair, let $B$ be an $\mathbb{R}$ divisor, and let $\pi: X \rightarrow S$ be a projective morphism between algebraic varieties. Let $H$ be an effective $\mathbb{R}$-Cartier $\mathbb{R}$-divisor on $X$ such that $K_{X}+B+H$ is $\pi$-nef and $(X, B+H)$ is lc. Then either $K_{X}+B$ is also $\pi$-nef, or there is a $\left(K_{X}+B\right)$-negative extremal ray $R$ such that $\left(K_{X}+B+\lambda H\right) \cdot R=0$, where

$$
\lambda:=\inf \left\{t \geq 0 \mid K_{X}+B+t H \text { is } \pi-n e f\right\} .
$$

Of course, $K_{X}+B+\lambda H$ is $\pi$-nef.

Proof. Assume that $K_{X}+B$ is not $\pi$-nef. Let $\left\{R_{j}\right\}$ be the set of $\left(K_{X}+B\right)$-negative extremal rays over $S$. Let $C_{j}$ be the rational curve spanning $R_{j}$ with the estimate as in Corollary 18.6 for every $j$. We put $\mu=\sup _{j}\left\{\mu_{j}\right\}$, where

$$
\mu_{j}=\frac{-\left(K_{X}+B\right) \cdot C_{j}}{H \cdot C_{j}} .
$$

Obviously, $\lambda=\mu$ and $0<\mu \leq 1$. So, it is sufficient to prove that $\mu=\mu_{l}$ for some $l$. By Corollary 18.6, there are positive real numbers $r_{1}, \ldots, r_{l}$ and a positive integer $m$, all independent of $j$, such that

$$
-\left(K_{X}+B\right) \cdot C_{j}=\sum_{i=1}^{l} \frac{r_{i} n_{i j}}{m}>0,
$$

where $n_{i j}$ is an integer with $n_{i j} \leq 2 m \operatorname{dim} X$ for every $i$ and $j$. If $\left(K_{X}+B+H\right) \cdot R_{l}$ $=0$ for some $l$, then there is nothing to prove since $\lambda=1$ and $\left(K_{X}+B+H\right) \cdot R$ $=0$ with $R=R_{l}$. Thus, we assume that $\left(K_{X}+B+H\right) \cdot R_{j}>0$ for every $j$. Therefore, we can apply Lemma 18.7 to obtain

$$
K_{X}+B+H=\sum_{p=1}^{q} r_{p}^{\prime}\left(K_{X}+\Delta_{p}\right),
$$

where $r_{1}^{\prime}, \ldots, r_{q}^{\prime}$ are positive real numbers, $\left(X, \Delta_{p}\right)$ is lc for every $p, m^{\prime}\left(K_{X}+\Delta_{p}\right)$ is Cartier for some positive integer $m^{\prime}$ and every $p$, and $\left(K_{X}+\Delta_{p}\right) \cdot C_{j}>0$ for 
every $p$ and $j$. So, we obtain

$$
\left(K_{X}+B+H\right) \cdot C_{j}=\sum_{p=1}^{q} \frac{r_{p}^{\prime} n_{p j}^{\prime}}{m^{\prime}}
$$

with $0<n_{p j}^{\prime}=m^{\prime}\left(K_{X}+\Delta_{p}\right) \cdot C_{j} \in \mathbb{Z}$. Note that $m^{\prime}$ and $r_{p}^{\prime}$ are independent of $j$ for every $p$. We also note that

$$
\frac{1}{\mu_{j}}=\frac{H \cdot C_{j}}{-\left(K_{X}+B\right) \cdot C_{j}}=\frac{\left(K_{X}+B+H\right) \cdot C_{j}}{-\left(K_{X}+B\right) \cdot C_{j}}+1=\frac{m \sum_{p=1}^{q} r_{p}^{\prime} n_{p j}^{\prime}}{m^{\prime} \sum_{i=1}^{l} r_{j} n_{i j}}+1 .
$$

Since

$$
\sum_{i=1}^{l} \frac{r_{i} n_{i j}}{m}>0
$$

for every $j$ and $n_{i j} \leq 2 m \operatorname{dim} X$ with $n_{i j} \in \mathbb{Z}$ for every $i$ and $j$, the cardinality of the set $\left\{n_{i j}\right\}_{i, j}$ is finite. Thus,

$$
\inf _{j}\left\{1 / \mu_{j}\right\}=1 / \mu_{l}
$$

for some $l$. Therefore, $\mu=\mu_{l}$, which finishes the proof.

The following picture helps the reader understand Theorem 18.9.

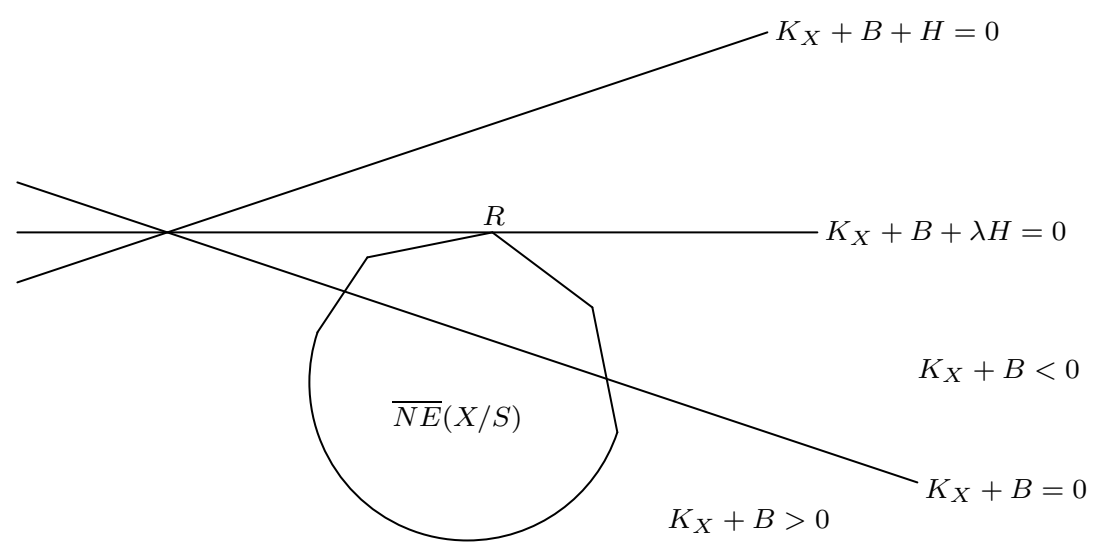

The main result of this section is an estimate of lengths of extremal rays which are relatively ample at non-lc loci (cf. [Ko2], [Ko3]).

Theorem 18.10. Let $X$ be a normal variety, let $B$ be an effective $\mathbb{R}$-divisor on $X$ such that $K_{X}+B$ is $\mathbb{R}$-Cartier, and let $\pi: X \rightarrow S$ be a projective morphism onto a variety $S$. Let $R$ be a $\left(K_{X}+B\right)$-negative extremal ray of $\overline{N E}(X / S)$ which is 
relatively ample at $\operatorname{Nlc}(X, B)$. Then we can find a rational curve $C$ on $X$ such that $[C] \in R$ and

$$
0<-\left(K_{X}+B\right) \cdot C \leq 2 \operatorname{dim} X
$$

Proof. By shrinking $S$, we can assume that $S$ is quasi-projective. By replacing $\pi: X \rightarrow S$ with the extremal contraction $\varphi_{R}: X \rightarrow Y$ over $S$ (cf. Theorem 16.6(3)), we can assume that the relative Picard number $\rho(X / S)$ is 1 and $\pi$ is an isomorphism in a neighborhood of $\operatorname{Nlc}(X, B)$. In particular, $-\left(K_{X}+B\right)$ is $\pi$-ample. By Theorem 10.4, there is a projective birational morphism $f: Y \rightarrow X$ such that

(i) $K_{Y}+B_{Y}=f^{*}\left(K_{X}+B\right)+\sum_{a(E, X, B)<-1}(a(E, X, B)+1) E$, where

$$
B_{Y}=f_{*}^{-1} B+\sum_{E: f \text {-exceptional }} E,
$$

(ii) $\left(Y, B_{Y}\right)$ is a $\mathbb{Q}$-factorial dlt pair, and

(iii) $D=B_{Y}+F$ with $F=-\sum_{a(E, X, B)<-1}(a(E, X, B)+1) E \geq 0$.

We note that $K_{Y}+D=f^{*}\left(K_{X}+B\right)$. Therefore,

$$
f_{*}\left(\overline{N E}(Y / S)_{K_{Y}+D \geq 0}\right) \subseteq \overline{N E}(X / S)_{K_{X}+B \geq 0}=\{0\} .
$$

We also note that

$$
f_{*}\left(\overline{N E}(Y / S)_{\mathrm{Nlc}(Y, D)}\right)=\{0\} .
$$

Thus, there is a $\left(K_{Y}+D\right)$-negative extremal ray $R^{\prime}$ of $\overline{N E}(Y / S)$ which is relatively ample at $\operatorname{Nlc}(Y, D)$. By Theorem 16.6(1), $R^{\prime}$ is spanned by a curve $C^{\dagger}$. Since $-\left(K_{Y}+D\right) \cdot C^{\dagger}>0$, we see that $f\left(C^{\dagger}\right)$ is a curve. If $C^{\dagger} \subset \operatorname{Supp} F$, then $f\left(C^{\dagger}\right) \subset$ $\operatorname{Nlc}(X, B)$. This is a contradiction because $\pi \circ f\left(C^{\dagger}\right)$ is a point. Thus, $C^{\dagger} \not \subset \operatorname{Supp} F$. Since $-\left(K_{Y}+B_{Y}\right)=-\left(K_{Y}+D\right)+F$, we can see that $R^{\prime}$ is a $\left(K_{Y}+B_{Y}\right)$-negative extremal ray of $\overline{N E}(Y / S)$. Therefore, we can find a rational curve $C^{\prime}$ on $Y$ such that $C^{\prime}$ spans $R^{\prime}$ and

$$
0<-\left(K_{Y}+B_{Y}\right) \cdot C^{\prime} \leq 2 \operatorname{dim} X
$$

by Theorem 18.2. By the above argument, we can easily see that $C^{\prime} \not \subset \operatorname{Supp} F$. Therefore, we obtain

$0<-\left(K_{Y}+D\right) \cdot C^{\prime}=-\left(K_{Y}+B_{Y}\right) \cdot C^{\prime}-F \cdot C^{\prime} \leq-\left(K_{Y}+B_{Y}\right) \cdot C^{\prime} \leq 2 \operatorname{dim} X$.

Since $K_{Y}+D=f^{*}\left(K_{X}+B\right)$, it follows that $C=f\left(C^{\prime}\right)$ is a rational curve on $X$ such that $\pi(C)$ is a point and $0<-\left(K_{X}+B\right) \cdot C \leq 2 \operatorname{dim} X$.

Remark 18.11. In Theorem 18.10, we can easily prove $0<-\left(K_{X}+B\right) \cdot C \leq$ $\operatorname{dim} X+1$ when $\operatorname{dim} X \leq 2$. For details, see [F17, Proposition 3.7]. 


\section{$\S 19$. Ambro's theory of quasi-log varieties}

In this section, we make some comments on Ambro's theory of quasi-log varieties. We recommend [F15] for an introduction to the theory of quasi-log varieties.

In the acknowledgements in [A1], Ambro wrote "The motivation behind this work is his (Professor Shokurov's) idea that log varieties and their LCS loci should be treated on an equal footing." So, in the theory of quasi-log varieties, we have to treat highly reducible non-equidimensional varieties (see Example 19.2 below). Therefore, our approach in this paper is completely different from the theory of quasi-log varieties. We recommend the reader to compare our proof of the base point free theorem for projective lc surfaces in Section 2 with Ambro's proof (see, for example, [F15, Section 4]).

Let us explain some results of the theory of quasi-log varieties which cannot be covered by our approach.

19.1. Let $(X, B)$ be a projective $\log$ canonical pair and let $\left\{C_{i}\right\}$ be any set of lc centers of the pair $(X, B)$. We put $W=\bigcup C_{i}$ with the reduced scheme structure. Then $[W, \omega]$ is a qlc pair, where $\omega=\left.\left(K_{X}+B\right)\right|_{W}$. For the definition of qlc pairs, see [F11, Definition 3.29] or [F15, Definition 3.1].

Example 19.2. Let $V$ be a projective toric variety and let $D$ be the complement of the big torus. Then $(V, D)$ is $\log$ canonical and $K_{V}+D \sim 0$. In this case, every torus invariant closed subvariety $W$ of $V$ with $\omega=0$ is a qlc pair. In particular, $W$ is not necessarily pure-dimensional (cf. [F7, §5]).

We can prove the cone theorem for $[W, \omega]$.

Theorem 19.3 (Cone theorem). We have

$$
\overline{N E}(W)=\overline{N E}(W)_{\omega \geq 0}+\sum_{j} R_{j} .
$$

For the details, see [F11, 3.3.3 Cone Theorem]. We can also prove the base point free theorem.

Theorem 19.4 (Base point free theorem). Let $L$ be a nef Cartier divisor on $W$ such that $a L-\omega$ is ample for some $a>0$. Then $|m L|$ is base point free for $m \gg 0$.

See, for example, [F11, 3.3.1 Base point free theorem]. By these theorems, we have the following statement.

Theorem 19.5 (Contraction theorem). Let $F$ be an $\omega$-negative extremal face of $\overline{N E}(W)$. Then there is a contraction morphism $\varphi_{F}: W \rightarrow V$ with the following properties: 
(i) Let $C$ be an integral curve on $W$. Then $\varphi_{F}(C)$ is a point if and only if $[C] \in F$.

(ii) $\mathcal{O}_{V} \simeq\left(\varphi_{F}\right)_{*} \mathcal{O}_{W}$

(iii) Let $L$ be a line bundle on $W$ such that $L \cdot C=0$ for every curve $C$ with $[C] \in F$. Then there is a line bundle $L_{V}$ on $V$ such that $L \simeq \varphi_{F}^{*} L_{V}$.

For the details of the theory of quasi-log varieties, see [F11]. The book [F11] also deals with various other topics which cannot be covered by this paper.

\section{§20. Related topics}

In this final section, for the reader's convenience we briefly discuss some related topics considered by the author in other publications.

In this paper, we did not describe the notion of singularities of pairs. However, it is very important in some papers on the log minimal model program. We think that $[\mathrm{F} 6]$ helps the reader understand the subtlety of the notion of dlt pairs.

The reader can find that all the injectivity, vanishing, and torsion-free theorems of this paper are discussed in full generality in [F11, Sections 2 and 3]. They heavily depend on the theory of mixed Hodge structures on compact support cohomology groups of reducible varieties.

We omitted the explanation of the log minimal model program for log canonical pairs. It is because the framework is the same as for klt pairs. The reader can find it in [F11, Section 3]. We note that the existence of log canonical flips is still an open problem in dimension $\geq 5$ and the termination of log canonical flips follows from the termination of klt flips. For the details, see [F11, Section 3].

In [F8], we prove an effective version of the base point free theorem for log canonical pairs. It is a log canonical version of Kollár's effective freeness. In [F9], the Angehrn-Siu type effective base point free theorems are proved for log canonical pairs. The reader can find that the proof of our non-vanishing theorem (cf. Theorem 12.1 and [F16, Theorem 1.1]) grew out from the arguments in [F8] and [F9].

In [F10], we systematically investigate the basic properties of non-lc ideal sheaves, especially, the restriction theorem of non-lc ideal sheaves for normal divisors. It is a generalization of Kawakita's inversion of adjunction on log canonicity. See also [FST] for a further discussion of various analogues of non-lc ideal sheaves.

In [F13], we prove the finite generation of the log canonical ring for log canonical pairs in dimension four and discuss related topics. The finite generation theorem implies the existence of fourfold log canonical flips.

In [F17], we discuss the minimal model theory for log surfaces. The results in [F17] are obtained under much weaker assumptions than the usual minimal model 
theory. The paper [F17] is an ultimate application of our new approach to the log minimal model program.

\section{Acknowledgements}

The author was partially supported by The Inamori Foundation and by the Grantin-Aid for Young Scientists (A) $\sharp 20684001$ from JSPS. He thanks Natsuo Saito for drawing a beautiful picture of a Kleiman-Mori cone. He also thanks Takeshi Abe for useful discussions and Yoshinori Gongyo for some questions. Finally, he thanks Professor Shigefumi Mori for useful comments, information, and warm encouragement.

\section{References}

[A1] F. Ambro, Quasi-log varieties, Tr. Mat. Inst. Steklova 240 (2003), 220-239 (in Russian); English transl.: Proc. Steklov Inst. Math. 240 (2003), 214-233. Zbl 1081.14021 MR 1993751

[A2] Non-klt techniques, in Flips for 3-folds and 4-folds, Oxford Lecture Ser. Math. Appl. 35, Oxford Univ. Press, 2007, 163-170. MR 2359347

[B1] C. Birkar, On existence of log minimal models, Compos. Math. 146 (2010), 919-928. Zbl 1197.14011 MR 2660678

[B2] _ On existence of log minimal models II, J. Reine Angew. Math., to appear.

$[\mathrm{BCHM}]$ C. Birkar, P. Cascini, C. Hacon, and J. McKernan, Existence of minimal models for varieties of log general type, J. Amer. Math. Soc. 23, 405-468. Z Zbl 1210.14019 MR 2601039

[BP] C. Birkar and M. Păun, Minimal models, flips and finite generation: a tribute to V. V. Shokurov and Y.-T. Siu, in Classification of algebraic varieties, EMS Ser. Congr. Rep., Eur. Math. Soc., Zürich, 2011, 77-113. Zbl pre05885267 MR 2779468

[D] P. Deligne, Théorie de Hodge. II, Inst. Hautes Études Sci. Publ. Math. 40 (1971), 5-57. Zbl 0219.14007 MR 0498551

[EV] H. Esnault and E. Viehweg, Lectures on vanishing theorems, DMV Seminar 20 Birkhäuser, Basel, 1992. Zbl 0779.14003 MR 1193913

[F1] O. Fujino, Abundance theorem for semi log canonical threefolds, Duke Math. J. 102 (2000), 513-532. Zbl 0986.14007 MR 1756108

[F2] Notes on toric varieties from Mori theoretic viewpoint, Tohoku Math. J. 55 (2003), 551-564. Zbl 1078.14077 MR 2017225

[F3] Higher direct images of log canonical divisors, J. Differential Geom. 66 (2004), 453-479. Zbl 1072.14019 MR 2106473

[F4] $\quad$ On the Kleiman-Mori cone, Proc. Japan Acad. Ser. A Math. Sci. 81 (2005), 80-84. Zbl 1093.14025 MR 1712649

[F5] _ Equivariant completions of toric contraction morphisms, Tohoku Math. J. 58 (2006), 303-321. Zbl 1127.14047 MR 2273272

[F6] What is log terminal?, in Flips for 3-folds and 4-folds, Oxford Lecture Ser. Math. Appl. 35, Oxford Univ. Press, 2007, 29-62. MR 2359341 
[F7] Vanishing theorems for toric polyhedra, in Higher dimensional algebraic varieties and vector bundles, RIMS Kokyuroku Bessatsu B9, RIMS, Kyoto, 2008, 81-95. Zbl pre05722827 MR 2509693

[F8] Effective base point free theorem for log canonical pairs-Kollár type theorem, Tohoku Math. J. 61 (2009), 475-481. Zbl 1189.14025 MR 2598245

[F9] _ Effective base point free theorem for log canonical pairs, II. Angehrn-Siu type theorems, Michigan Math. J. 59 (2010), 303-312. Zbl 1201.14010 MR 2677623

[F10] Theory of non-lc ideal sheaves: basic properties, Kyoto J. Math. 50 (2010), 225-245. Zbl 1200.14033 MR 2666656

[F11] Introduction to the log minimal model program for log canonical pairs, arXiv:0907.1506v1, 2009 .

[F12] On injectivity, vanishing and torsion-free theorems for algebraic varieties, Proc. Japan Acad. Ser. A Math. Sci. 85 (2009), 95-100. Zbl 1189.14024 MR 2561896

[F13] Finite generation of the log canonical ring in dimension four, Kyoto J. Math. 50 (2010), 671-684. Zbl 1210.14020 MR 2740690

[F14] On Kawamata's theorem, in Classification of algebraic varieties, EMS Ser. Congr. Rep., Eur. Math. Soc., Zürich, 2011, 305-315. Zbl 1213.14015 MR 2779478

[F15] Introduction to the theory of quasi-log varieties, ibid., 289-303. Zbl 1213.14030 MR 2779477

[F16] _ Non-vanishing theorem for log canonical pairs, J. Algebraic Geom., to appear.

[F17] Minimal model theory for log surfaces, preprint, 2010.

[F18] Semi-stable minimal model program for varieties with trivial canonical divisor, Proc. Japan Acad. Ser. A Math. Sci. 87 (2011), 25-30.

[FG] O. Fujino and Y. Gongyo, On canonical bundle formulae and subadjunctions, preprint, 2010 .

[FP] O. Fujino and S. Payne, Smooth complete toric threefolds with no nontrivial nef line bundles, Proc. Japan Acad. Ser. A Math. Sci. 81 (2005), 174-179. Zbl 1141.14313 MR 2196723

[FST] O. Fujino, K. Schwede, and S. Takagi, Supplements to non-lc ideal sheaves, in Higher dimensional algebraic geometry, RIMS Kokyuroku Bessatsu B24, RIMS, Kyoto, 2011, $1-46$.

[G] Y. Gongyo, On weak Fano varieties with log canonical singularities, J. Reine Angew. Math., to appear.

[HM] C. Hacon and J. McKernan, Extension theorems and the existence of flips, in Flips for 3-folds and 4-folds, Oxford Lecture Ser. Math. Appl. 35, Oxford Univ. Press, Oxford, 2007, 76-110. MR 2359343

[Ka1] Y. Kawamata, The cone of curves of algebraic varieties, Ann. of Math. (2) 119 (1984), 603-633. Zbl 0544.14009 MR 0744865

[Ka2] On the length of an extremal rational curve, Invent. Math. 105 (1991), 609611. Zbl 0751.14007 MR 1117153

[KMM] Y. Kawamata, K. Matsuda, and K. Matsuki, Introduction to the minimal model problem, in Algebraic geometry, Sendai 1985, Adv. Stud. Pure Math. 10, Kinokuniya and North-Holland, 1987, 283-360. Zbl 0672.14006 MR 0946243

[Kl] S. L. Kleiman, Toward a numerical theory of ampleness, Ann. of Math. (2) 84 (1966), 293-344. Zbl 0146.17001 MR 0206009

[Ko1] J. Kollár, The cone theorem, Ann. of Math. (2) 120 (1984), 1-5. Zbl 0544.14010 
MR 0750714

[Ko2] - Cone theorems and cyclic covers, in Algebraic geometry and analytic geometry (Tokyo, 1990), ICM-90 Satell. Conf. Proc., Springer, Tokyo, 1991, 101-110. Zbl 0807.14010 MR 1260941

[Ko3] Cone theorems and bug-eyed covers, J. Algebraic Geom. 1 (1992), 293-323. Zbl 0807.14009 MR 1144441

[Ko4] Shafarevich maps and automorphic forms, M. B. Porter Lectures, Princeton Univ. Press, Princeton, NJ, 1995. Zbl 0871.14015 MR 1341589

[KK] J. Kollár and S. Kovács, Log canonical singularities are Du Bois, J. Amer. Math. Soc. 23 (2010), 791-813. Zbl 1202.14003 MR 2629988

[KM] J. Kollár and S. Mori, Birational geometry of algebraic varieties, Cambridge Tracts in Math. 134, Cambridge Univ. Press., 1998. Zbl 0926.14003 MR 1658959

[L] R. Lazarsfeld, Positivity in algebraic geometry. II. Positivity for vector bundles, and multiplier ideals, Ergeb. Math. Grenzgeb. 49, Springer, Berlin, 2004. Zbl 1093.14500 MR 2095472

[Ma] K. Matsuki, Introduction to the Mori program, Universitext, Springer, New York, 2002. Zbl 0988.14007 MR 1875410

[Mo] S. Mori, Threefolds whose canonical bundles are not numerically effective, Ann. of Math. (2) 116 (1982), 133-176. Zbl 0557.14021 MR 0662120

[R] M. Reid, Projective morphisms according to Kawamata, preprint, 1983.

[S1] V. V. Shokurov, The nonvanishing theorem, Izv. Akad. Nauk SSSR Ser. Mat. 49 (1985), 635-651 (in Russian). Zbl 0605.14006 MR 0794958

[S2] _ Three-dimensional log perestroikas (with an appendix in English by Y. Kawamata), Izv. Ross. Akad. Nauk Ser. Mat. 56 (1992), 105-203 (in Russian); English transl.: Russian Acad. Sci. Izv. Math. 40 (1993), 95-202. MR 1162635

[S3] , 3-fold log models, in Algebraic geometry, 4, J. Math. Sci. 81 (1996), 2667-2699. Zbl 0873.14014 MR 1420223

[S4] Letters of a bi-rationalist: VII. Ordered termination, in Multidimensional algebraic geometry, Tr. Mat. Inst. Steklova 264 (2009), 184-208 (in Russian). MR 2590847 